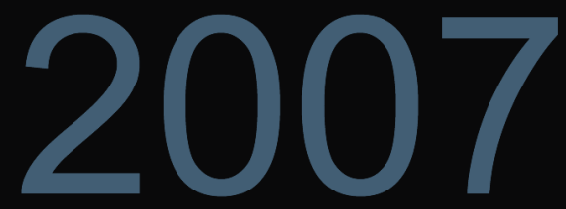

\title{
Idaho National Laboratory Annual Illness and Injury Surveillance Report
}

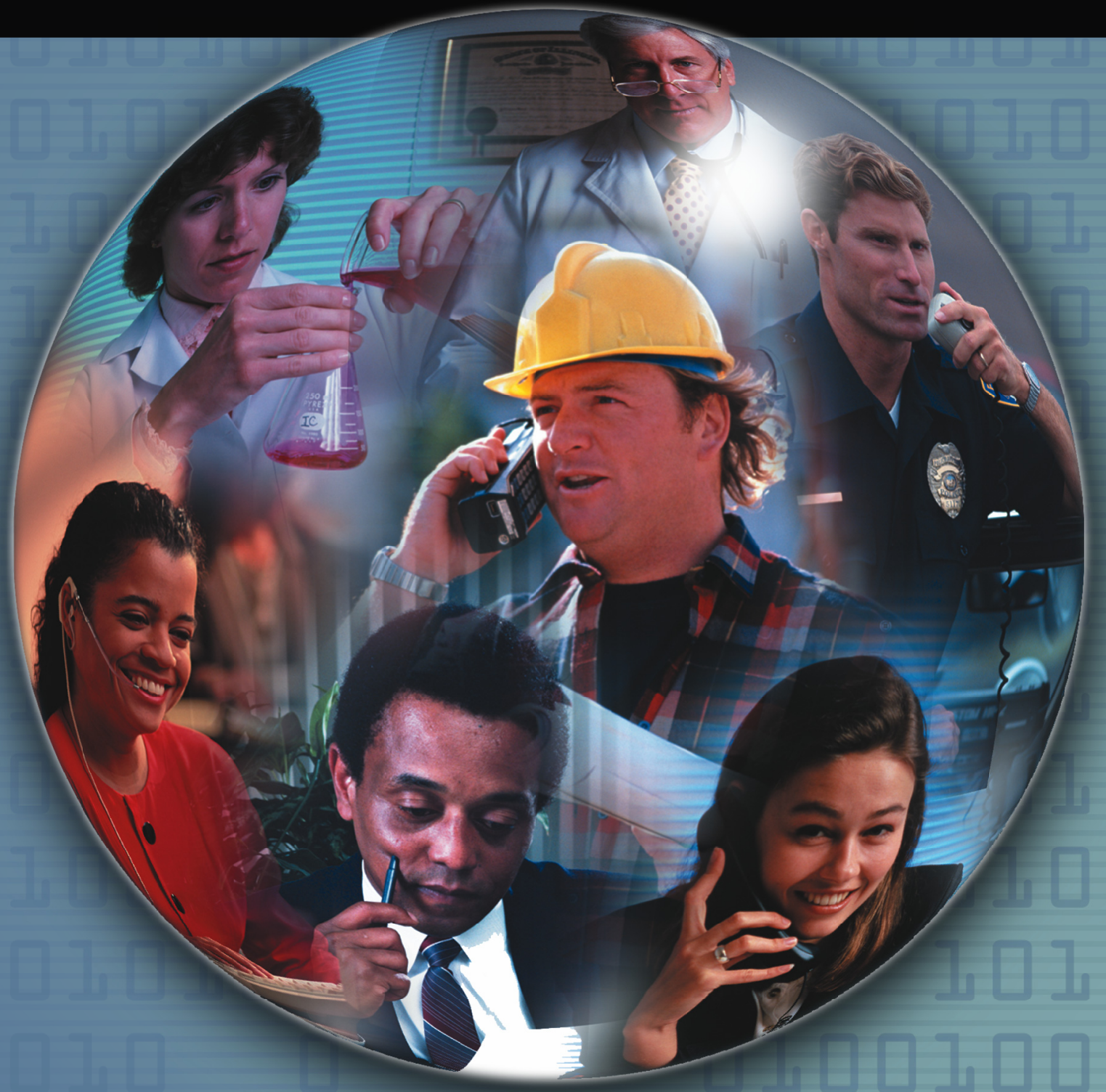




\title{
Idaho National Laboratory \\ 2007 Illness and Injury Surveillance Report
}

Questions or comments about this report or the Illness and Injury Surveillance Program (IISP) may be directed to:

E-mail:

Dr. Cliff Strader at cliff.strader@hq.doe.gov

or Dr. Bonnie Richter at bonnie.richter@hq.doe.gov

or direct letters to:

\author{
Mail Stop HS-13 / 270CC \\ U.S. Department of Energy \\ 1000 Independence Avenue, S.W. \\ Washington, DC 20585-0270
}

Additional information about the Department of Energy's Office of Illness and Injury Prevention Programs, the IISP, and annual reports for DOE sites participating in this program can be found at:

http://www.hss.energy.gov/healthsafety/WSHP/epi/surv/

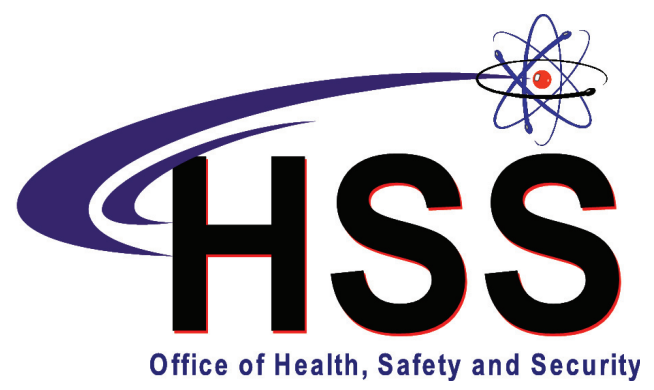

ACKNOWLEDGEMENT

LifeART images copyright 2000 Lippincott Williams \& Wilkins. All rights reserved.

This document was produced under contract number DE-AC05-06OR23100 between the

U.S. Department of Energy and Oak Ridge Associated Universities. 


\title{
Idaho National Laboratory 2007 Illness and Injury Surveillance Report
}

\author{
At A Glance
}

The 5,305 employees at Idaho National Laboratory (INL) in 2007 represented a 21 percent decrease from 2006. The INL work force has declined 37 percent since 2002, when there were 8,453 workers. The average age of the work force has increased slightly since 2002 for both men and women. In 2007, the average age was 48 years for men and 46 years for women, compared with the 2002 averages of 46 years and 44 years, respectively.

In keeping with the decreased work force, the total number of absences at INL decreased 34 percent from 2006 to 2007 . The average length of an absence at INL decreased during this same period from 58 days in 2006 to 36 days in 2007.

The absence rate among all workers decreased from 2.89 per 100 workers in 2006 to 2.43 per 100 workers in 2007. Absence rates have been relatively stable since 2004 .

In 2007, 21 absences among 21 women produced an absence rate of 1.56 per 100 workers $(21 / 1,349)$. A total of 108 absences reported by 103 men resulted in an absence rate of 2.73 per 100 workers $(108 / 3,956)$. The lower absence rate among women than among men is consistent with the absence rates observed in 2006. Disorders of the musculoskeletal system, injuries, and respiratory disorders were among the top 3 diagnoses reported by men and women at INL from 2003 to 2007.

Women reported 13 OSHA events and men reported 30 OSHA events at INL in 2007. A total of 47 diagnoses were reported for these events, mostly the result of injuries. 
The Idaho National Laboratory Work

Force - 2007

The Work Force by Gender and Age

The Work Force by Gender and Job

Category

\section{Number and Length of Absences}

Absence Rate by Gender and Age 2

Number of Days Absent by

Gender and Age

Absence Rate by Job Category

and Gender...

Average Duration of Absence by

Job Category and Gender.

\section{Diagnostic Categories}

Number of Diagnoses and Lost Calendar Days by Diagnostic Category (Categorized by ICD-9-CM) and Gender.... . .4

Common Diagnoses Among Female

Workers in 2007 $\ldots 5$

Common Diagnoses Among Male

Workers in 2007

Number of Most Frequently Reported Diagnoses by Job Category and Gender .....7

\section{Rates of Disease Occurrence}

Rates for All Illnesses and Injuries

Combined by Job Category, Gender, and Age

Rates for Selected Diagnostic Categories by Job Category, Gender, and Age. .8

\section{Time Trends}

Age-Adjusted Rates for All Diagnoses Combined Among Women and Men from 2003 to 2007 . 10
Age-Adjusted Rates for Selected Diagnostic Categories Among Women and Men from

2003 to 2007

Age-Adjusted Rates for All Diagnoses Combined Among Women and Men by Job Category from 2003 to 2007 .............. 12

\section{Sentinel Health Events for Occupations (SHEOs)}

Characteristics of SHEOs by Gender.... 13

SHEO Diagnoses by Gender.... 13

Occupational Safety and Health Administration (OSHA)-Recordable Events

OSHA-Recordable Events by Gender and Age 14

OSHA-Recordable Events by Job

Category and Gender .... 14

Diagnostic and Accident Categories for OSHA-Recordable Events

OSHA-Recordable Diagnoses by

Diagnostic Category and Gender.... 15

OSHA-Recordable Accidents by Type and Gender. 15

\section{Rates of OSHA-Recordable Events}

OSHA-Recordable Rates by Age and Job Categories Among Women, All

Diagnoses Combined 16

OSHA-Recordable Rates by Age and Job Categories Among Men, All Diagnoses Combined 16

Time Trends for OSHA-Recordable Events

Age-Adjusted Rates for All OSHA-Recordable Diagnoses Combined Among Women and Men by Job Category from 2003 to 2007 ... 17

\section{Appendices}

Appendices A-W. 19 
The Idaho National Laboratory Work Force - 2007

Figure 1. The Work Force by Gender and Age

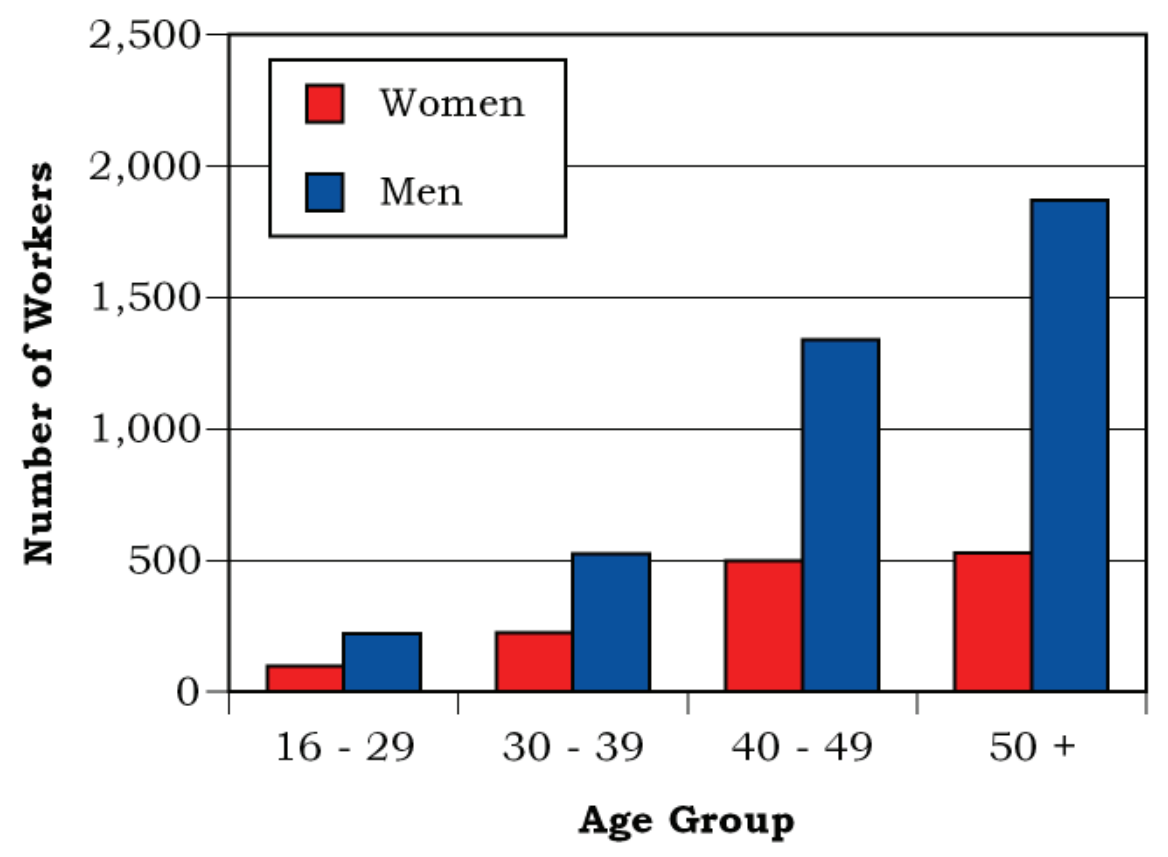

Figure 2. The Work Force by Gender and Job Category

\begin{tabular}{|c|c|c|}
\hline Job Category & Women & Men \\
\hline \multirow{2}{*}{ Professional } & 359 & 1,598 \\
\hline & $27 \%$ & $40 \%$ \\
\hline \multirow{2}{*}{ Administrative Support } & 681 & 712 \\
\hline & $51 \%$ & $18 \%$ \\
\hline \multirow{2}{*}{ Technical Support } & 124 & 554 \\
\hline & $\mathbf{9 \%}$ & $14 \%$ \\
\hline \multirow{2}{*}{ Service } & 96 & 271 \\
\hline & $7 \%$ & $7 \%$ \\
\hline \multirow{2}{*}{ Security and Fire } & 29 & 206 \\
\hline & $2 \%$ & $5 \%$ \\
\hline \multirow{2}{*}{ Crafts } & 43 & 456 \\
\hline & $3 \%$ & $12 \%$ \\
\hline \multirow{2}{*}{ Line Operators } & 17 & 159 \\
\hline & $1 \%$ & $4 \%$ \\
\hline \multirow[t]{2}{*}{ Total } & 1,349 & 3,956 \\
\hline & $100 \%$ & $100 \%$ \\
\hline
\end{tabular}


Number and Length of Absences

Figure 3. Absence Rate by Gender and Age

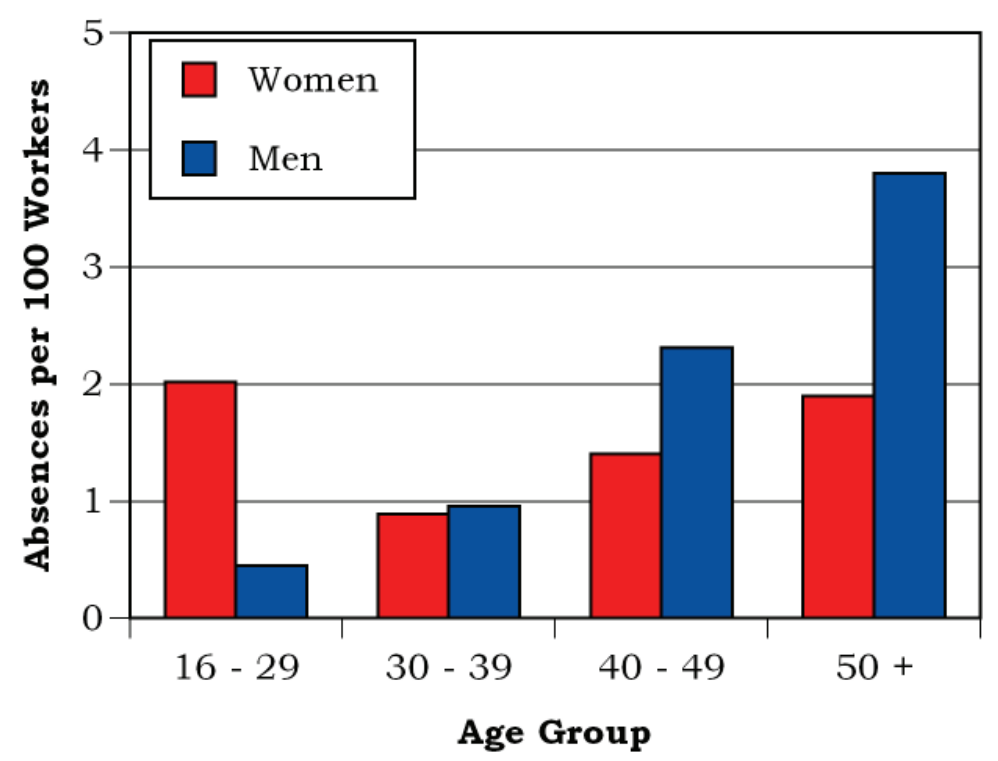

Figure 4. Number of Days Absent by Gender and Age

\begin{tabular}{|c|c|c|c|c|}
\hline \multirow{3}{*}{ Gender } & \multirow{2}{*}{ Age } & Number of & \multicolumn{2}{|c|}{ Number of Days Absent } \\
\cline { 3 - 5 } & & Absences & Total & Average \\
\hline \multirow{4}{*}{ Women } & $16-29$ & 2 & 21 & 11 \\
\cline { 2 - 5 } & $30-39$ & 2 & 56 & 28 \\
\cline { 2 - 5 } & $40-49$ & 7 & 121 & 17 \\
\cline { 2 - 5 } & $50+$ & 10 & 346 & 35 \\
\cline { 2 - 5 } & Total & 21 & 544 & 26 \\
\hline \multirow{4}{*}{ Men } & $16-29$ & 1 & 16 & 16 \\
\cline { 2 - 5 } & $30-39$ & 5 & 135 & 27 \\
\cline { 2 - 5 } & $40-49$ & 31 & 1,307 & 42 \\
\cline { 2 - 5 } & $50+$ & 71 & 2,697 & 38 \\
\cline { 2 - 5 } & Total & 108 & 4,155 & 38 \\
\hline
\end{tabular}


Figure 5. Absence Rate by Job Category and Gender

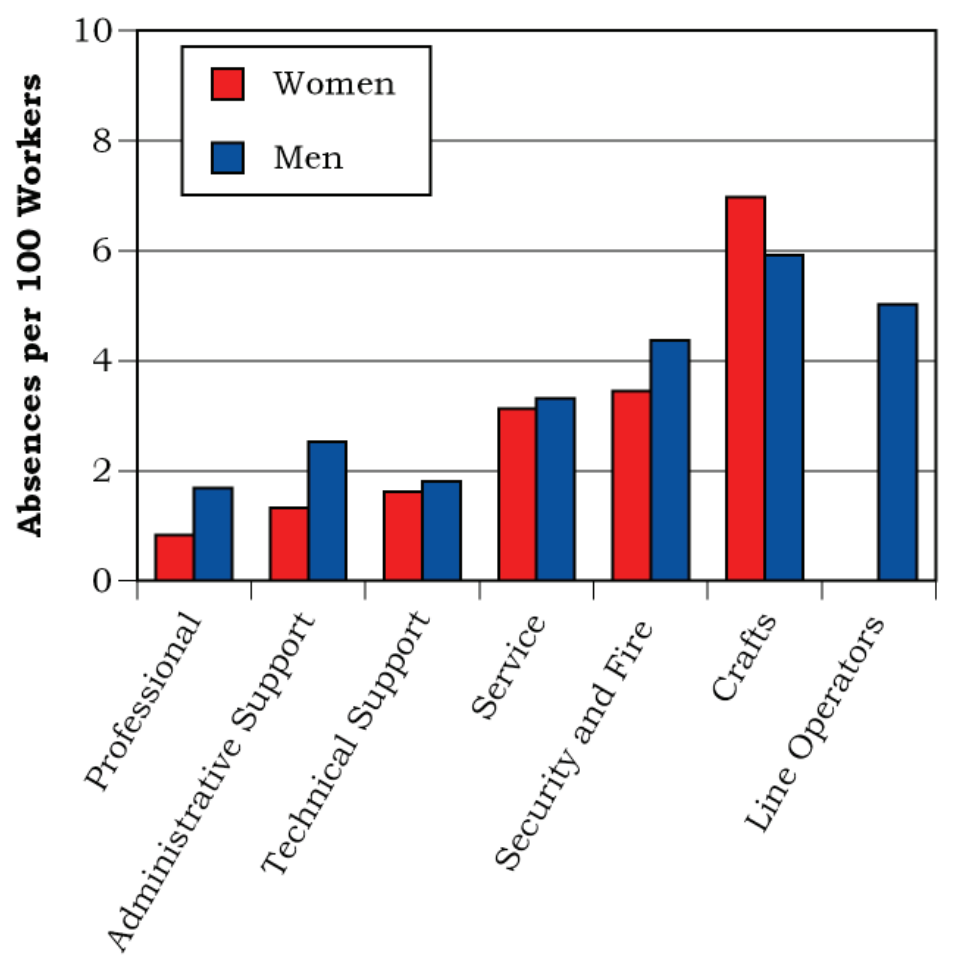

Job Category

Figure 6. Average Duration of Absence by Job Category and Gender

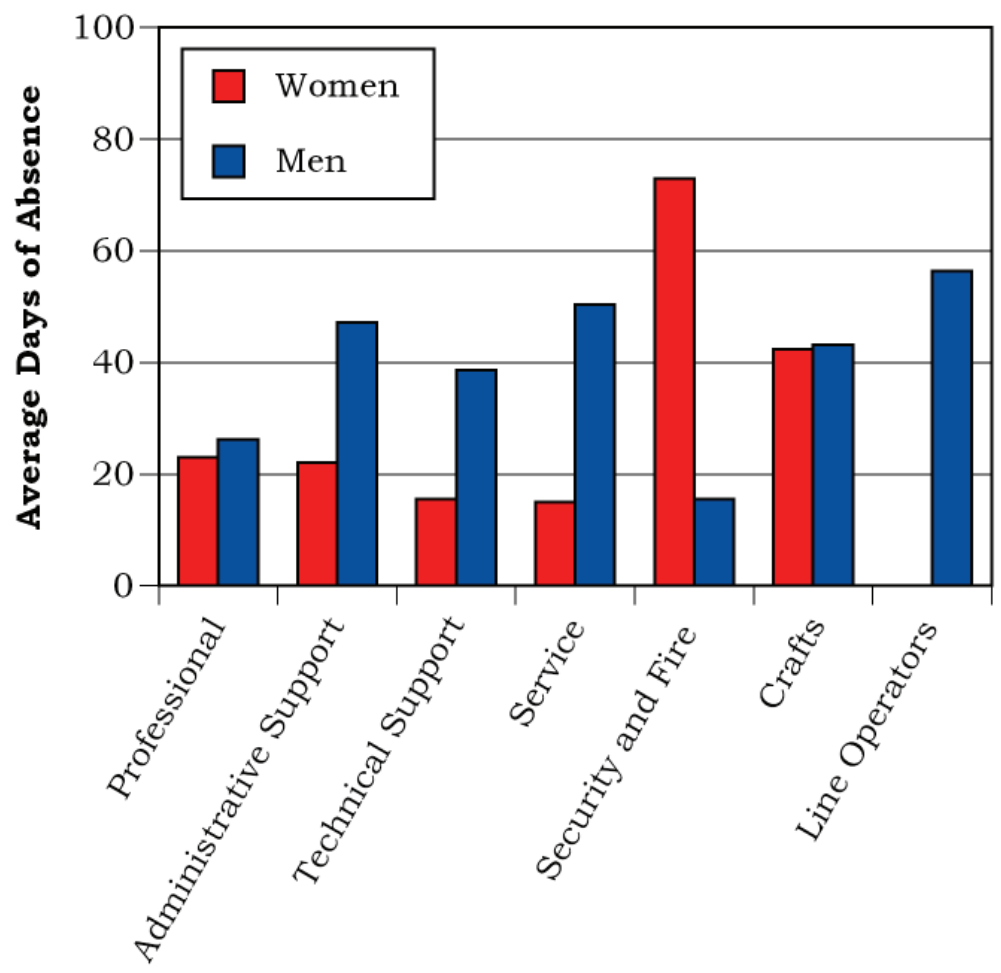

Job Category 


\section{Diagnostic Categories}

Figure 7. Number of Diagnoses and Lost Calendar Days by Diagnostic Category (Categorized by ICD-9-CM) and Gender

\begin{tabular}{|c|c|c|c|c|}
\hline \multirow[b]{2}{*}{ Diagnostic Category } & \multicolumn{2}{|c|}{ Women } & \multicolumn{2}{|c|}{ Men } \\
\hline & $\begin{array}{l}\text { Number of } \\
\text { Diagnoses }\end{array}$ & $\begin{array}{c}\text { Number } \\
\text { of Lost } \\
\text { Calendar } \\
\text { Days }\end{array}$ & $\begin{array}{l}\text { Number of } \\
\text { Diagnoses }\end{array}$ & $\begin{array}{c}\text { Number } \\
\text { of Lost } \\
\text { Calendar } \\
\text { Days }\end{array}$ \\
\hline Benign Growths & 0 & 0 & 1 & 26 \\
\hline Blood & 0 & 0 & 0 & 0 \\
\hline Cancer & 1 & 91 & 4 & 174 \\
\hline Digestive & 5 & 50 & 17 & 536 \\
\hline Endocrine/Metabolic & 0 & 0 & 2 & 39 \\
\hline Existing Birth Condition & 0 & 0 & 0 & 0 \\
\hline Genitourinary & 2 & 41 & 4 & 65 \\
\hline Heart/Circulatory & 1 & 43 & 14 & 738 \\
\hline Infections / Parasites & 0 & 0 & 2 & 45 \\
\hline Injury & 5 & 170 & 28 & 834 \\
\hline Miscarriage & 0 & 0 & NA & NA \\
\hline Musculoskeletal & 3 & 63 & 30 & 1,416 \\
\hline Nervous System & 1 & 20 & 5 & 113 \\
\hline Psychological & 0 & 0 & 6 & 142 \\
\hline Respiratory & 8 & 92 & 21 & 440 \\
\hline Skin & 0 & 0 & 1 & 33 \\
\hline Unspecified Symptoms & 0 & 0 & 3 & 196 \\
\hline
\end{tabular}

Note: Lost calendar days for each absence are counted more than once when multiple diagnoses occur in different diagnostic categories for the same absence. 
Figure 8. Common Diagnoses Among Female Workers in 2007

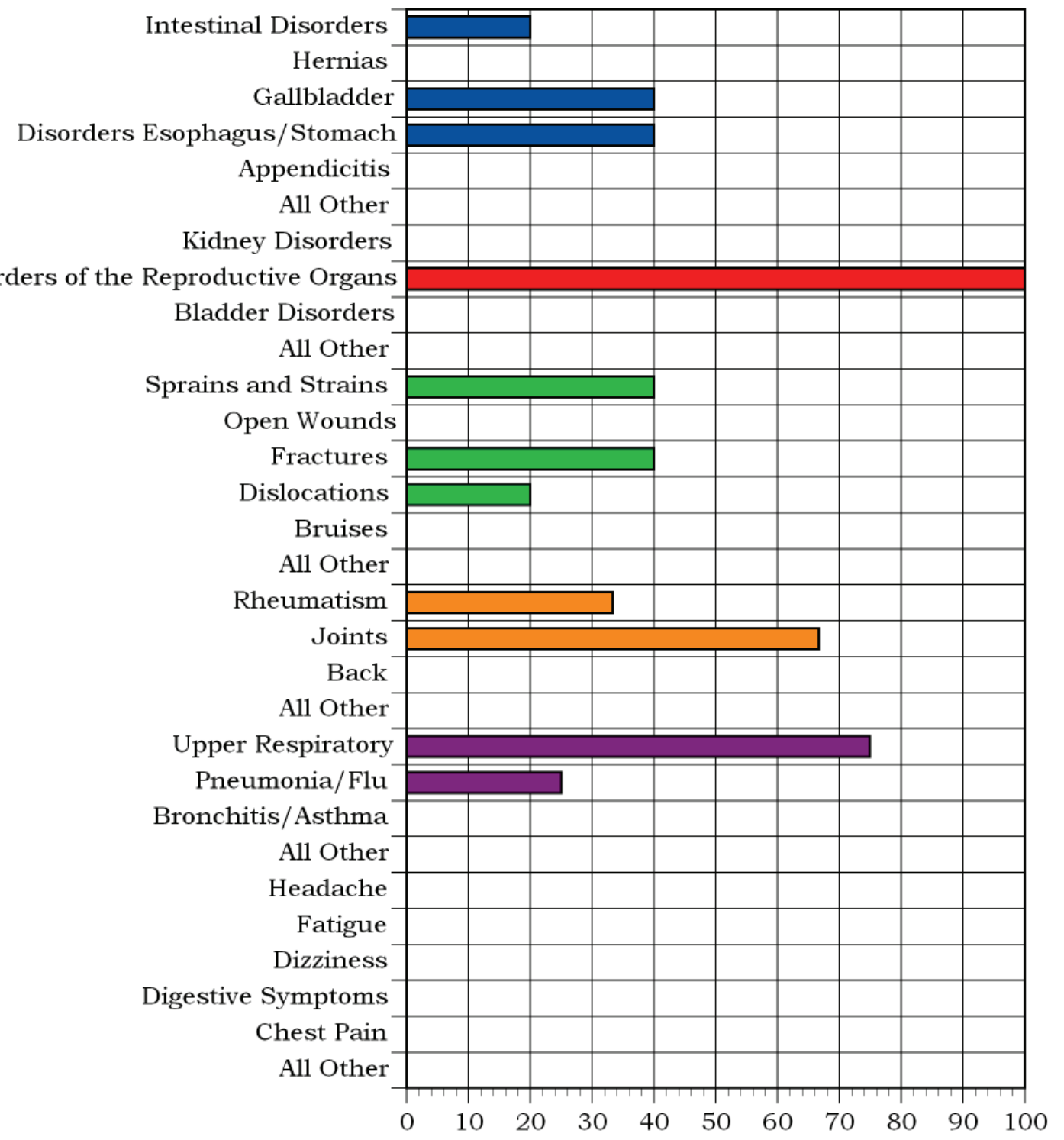

Percent Distribution of Diagnoses Within Diagnostic Category

\begin{tabular}{lll}
$\square$ Digestive, 5 Diagnoses & $\square$ Musculoskeletal, 3 Diagnoses \\
$\square$ Genitourinary, 2 Diagnoses & $\square$ Respiratory, 8 Diagnoses \\
$\square$ Injury, 5 Diagnoses & $\square$ Unspecified Symptoms, 0 Diagnoses \\
\hline
\end{tabular}


Figure 9. Common Diagnoses Among Male Workers in 2007

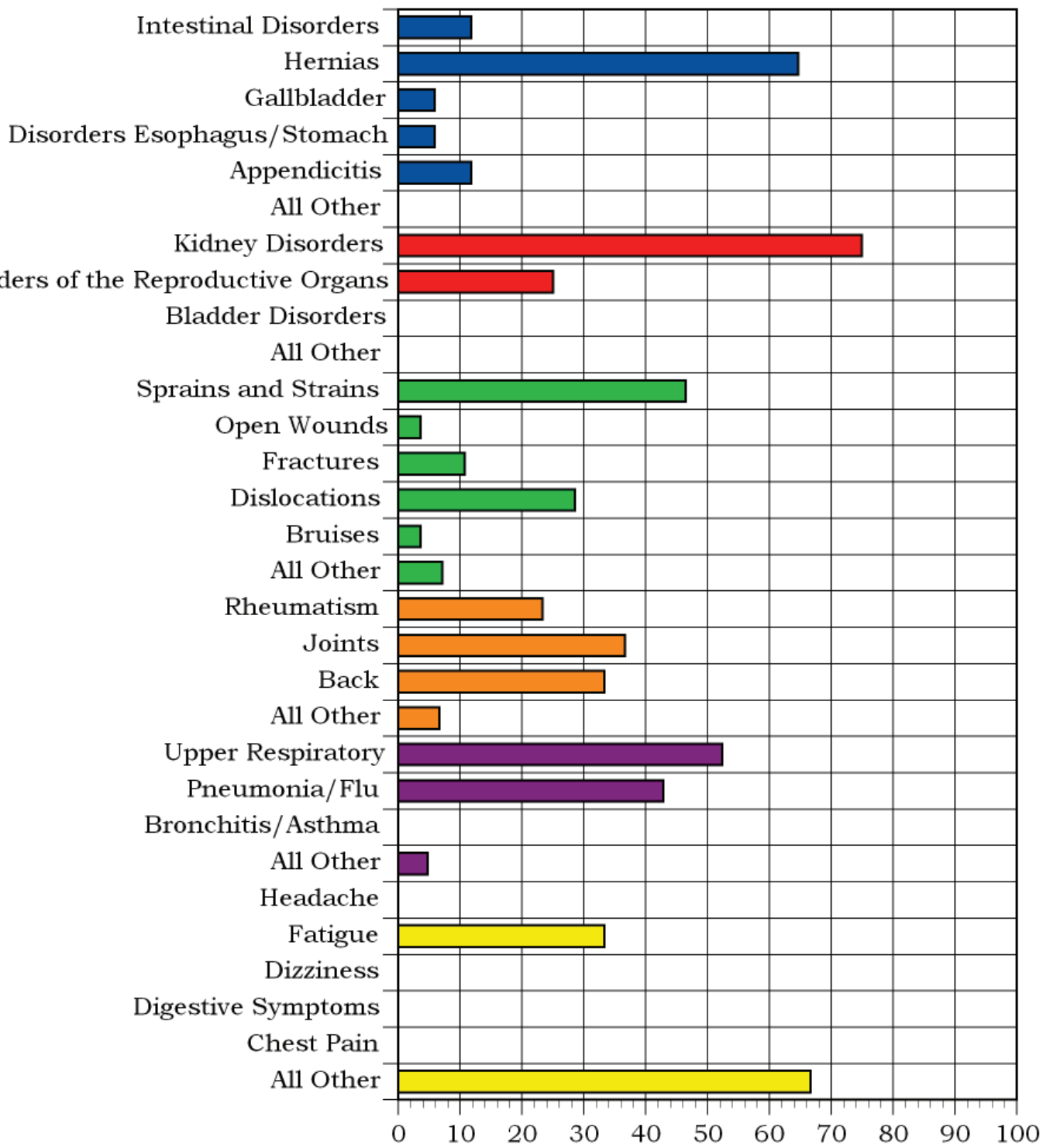

Percent Distribution of Diagnoses Within Diagnostic Category

Digestive, 17 Diagnoses

Genitourinary, 4 Diagnoses

Injury, 28 Diagnoses
Musculoskeletal, 30 Diagnoses

Respiratory, 21 Diagnoses

Unspecified Symptoms, 3 Diagnoses 


\section{Figure 10. Number of Most Frequently Reported Diagnoses by Job Category and Gender}

\begin{tabular}{|c|c|c|c|c|}
\hline Job Category & \multicolumn{2}{|l|}{ Men } & \multicolumn{2}{|l|}{ Women } \\
\hline \multirow{3}{*}{ Professional } & Injury & 9 & Genitourinary & 1 \\
\hline & Musculoskeletal & 7 & Injury & 1 \\
\hline & Respiratory & 7 & Respiratory & 1 \\
\hline \multirow{6}{*}{ Administrative Support } & Musculoskeletal & 7 & Respiratory & 4 \\
\hline & Respiratory & 6 & Musculoskeletal & 3 \\
\hline & Digestive & 4 & Digestive & 1 \\
\hline & & & Genitourinary & 1 \\
\hline & & & Heart/Circulatory & 1 \\
\hline & & & Injury & 1 \\
\hline \multirow{3}{*}{ Technical Support } & Injury & 4 & Digestive & 2 \\
\hline & Digestive & 2 & Respiratory & 1 \\
\hline & Respiratory & 2 & & \\
\hline \multirow{4}{*}{ Service } & Musculoskeletal & 4 & Digestive & 1 \\
\hline & Genitourinary & 2 & Injury & 1 \\
\hline & Heart/Circulatory & 2 & Nervous System & 1 \\
\hline & Injury & 2 & Respiratory & 1 \\
\hline \multirow{4}{*}{ Security and Fire } & Heart/Circulatory & 4 & Injury & 1 \\
\hline & Respiratory & 3 & & \\
\hline & Injury & 1 & & \\
\hline & Musculoskeletal & 1 & & \\
\hline \multirow{6}{*}{ Crafts } & Musculoskeletal & 9 & Cancer & 1 \\
\hline & Injury & 8 & Digestive & 1 \\
\hline & Digestive & 3 & Injury & 1 \\
\hline & Heart/Circulatory & 3 & Respiratory & 1 \\
\hline & Nervous System & 3 & & \\
\hline & Psychological & 3 & & \\
\hline \multirow{7}{*}{ Line Operators } & Digestive & 4 & & $\mathbf{0}$ \\
\hline & Musculoskeletal & 2 & & \\
\hline & Cancer & 1 & & \\
\hline & Heart/Circulatory & 1 & & \\
\hline & Injury & 1 & & \\
\hline & Respiratory & 1 & & \\
\hline & Unspecified Symptoms & 1 & & \\
\hline
\end{tabular}




\section{Rates of Disease Occurrence}

Figure 11. Rates for All Illnesses and Injuries Combined by Job Category, Gender, and Age

\begin{tabular}{|c|c|c|c|c|}
\hline \multirow{2}{*}{$\begin{array}{c}\text { All Illnesses \& } \\
\text { Injuries Combined }\end{array}$} & \multicolumn{4}{|c|}{ Rate per 1,000} \\
\hline & Job Category & Age & Men & Women \\
\hline & \multirow{2}{*}{ Professional } & $<50$ & 10 & 8 \\
\hline & & $50+$ & 34 & 9 \\
\hline & \multirow{2}{*}{ Administrative Support } & $<50$ & 14 & 16 \\
\hline & & $50+$ & 61 & 17 \\
\hline & \multirow{2}{*}{ Technical Support } & $<50$ & 19 & 23 \\
\hline & & $50+$ & 26 & 26 \\
\hline & \multirow{2}{*}{ Service } & $<50$ & 17 & 41 \\
\hline & & $50+$ & 67 & 43 \\
\hline & \multirow{2}{*}{ Security and Fire } & $<50$ & 24 & 0 \\
\hline & & $50+$ & 75 & 77 \\
\hline & \multirow{2}{*}{ Crafts } & $<50$ & 58 & 107 \\
\hline & & $50+$ & 90 & 67 \\
\hline & \multirow{2}{*}{ Line Operators } & $<50$ & 34 & 0 \\
\hline & & $50+$ & 113 & 0 \\
\hline
\end{tabular}

Figure 12. Rates for Selected Diagnostic Categories by Job Category, Gender, and Age

\begin{tabular}{|c|c|c|c|c|}
\hline \multirow{2}{*}{ Cancer } & \multicolumn{4}{|c|}{ Rate per 1,000} \\
\hline & Job Category & Age & Men & Women \\
\hline \multirow{14}{*}{ E. } & \multirow{2}{*}{ Professional } & $<50$ & 0 & 0 \\
\hline & & $50+$ & 1 & 0 \\
\hline & \multirow{2}{*}{ Administrative Support } & $<50$ & 3 & 0 \\
\hline & & $50+$ & 3 & 0 \\
\hline & \multirow{2}{*}{ Technical Support } & $<50$ & 0 & 0 \\
\hline & & $50+$ & 0 & 0 \\
\hline & \multirow{2}{*}{ Service } & $<50$ & 0 & 0 \\
\hline & & $50+$ & 0 & 0 \\
\hline & \multirow{2}{*}{ Security and Fire } & $<50$ & 0 & 0 \\
\hline & & $50+$ & 0 & 0 \\
\hline & \multirow{2}{*}{ Crafts } & $<50$ & 0 & 0 \\
\hline & & $50+$ & 0 & 67 \\
\hline & \multirow{2}{*}{ Line Operators } & $<50$ & 0 & 0 \\
\hline & & $50+$ & 14 & 0 \\
\hline
\end{tabular}




\section{Figure 12. Rates for Selected Diagnostic Categories by Job Category, Gender, and Age (Continued)}

\begin{tabular}{|c|c|c|c|c|}
\hline \multirow{2}{*}{ Heart/Circulatory } & \multicolumn{4}{|c|}{ Rate per 1,000} \\
\hline & Job Category & Age & Men & Women \\
\hline & \multirow{2}{*}{ Professional } & $<50$ & 0 & 0 \\
\hline & & $50+$ & 1 & 0 \\
\hline & \multirow{2}{*}{ Administrative Support } & $<50$ & 0 & 3 \\
\hline & & $50+$ & 6 & 0 \\
\hline & \multirow{2}{*}{ Technical Support } & $<50$ & 0 & 0 \\
\hline & & $50+$ & 4 & 0 \\
\hline & \multirow{2}{*}{ Service } & $<50$ & 0 & 0 \\
\hline & & $50+$ & 13 & 0 \\
\hline & \multirow{2}{*}{ Security and Fire } & $<50$ & 0 & 0 \\
\hline & & $50+$ & 50 & 0 \\
\hline & \multirow{2}{*}{ Crafts } & $<50$ & 0 & 0 \\
\hline & & $50+$ & 15 & 0 \\
\hline & \multirow{2}{*}{ Line Operators } & $<50$ & 0 & 0 \\
\hline & & $50+$ & 14 & 0 \\
\hline
\end{tabular}

\begin{tabular}{|c|c|c|c|c|}
\hline \multirow{2}{*}{ Respiratory } & \multicolumn{4}{|c|}{ Rate per 1,000} \\
\hline & Job Category & Age & Men & Women \\
\hline & \multirow{2}{*}{ Professional } & $<50$ & 2 & 4 \\
\hline & & $50+$ & 6 & 0 \\
\hline & \multirow{2}{*}{ Administrative Support } & $<50$ & 5 & 8 \\
\hline & & $50+$ & 12 & 3 \\
\hline & \multirow{2}{*}{ Technical Support } & $<50$ & 3 & 0 \\
\hline & & $50+$ & 4 & 26 \\
\hline & \multirow{2}{*}{ Service } & $<50$ & 0 & 20 \\
\hline & & $50+$ & 0 & 0 \\
\hline & \multirow{2}{*}{ Security and Fire } & $<50$ & 8 & 0 \\
\hline & & $50+$ & 25 & 0 \\
\hline & \multirow{2}{*}{ Crafts } & $<50$ & 4 & 36 \\
\hline & & $50+$ & 5 & 0 \\
\hline & \multirow{2}{*}{ Line Operators } & $<50$ & 11 & 0 \\
\hline & & $50+$ & 0 & 0 \\
\hline
\end{tabular}


Figure 12. Rates for Selected Diagnostic Categories by Job Category, Gender, and Age (Continued)

\begin{tabular}{|c|c|c|c|c|}
\hline \multirow{2}{*}{ Injury } & \multicolumn{4}{|c|}{ Rate per 1,000} \\
\hline & Job Category & Age & Men & Women \\
\hline \multirow{14}{*}{1} & \multirow{2}{*}{ Professional } & $<50$ & 6 & 0 \\
\hline & & $50+$ & 5 & 9 \\
\hline & \multirow{2}{*}{ Administrative Support } & $<50$ & 0 & 0 \\
\hline & & $50+$ & 9 & 3 \\
\hline & \multirow{2}{*}{ Technical Support } & $<50$ & 3 & 0 \\
\hline & & $50+$ & 13 & 0 \\
\hline & \multirow{2}{*}{ Service } & $<50$ & 8 & 0 \\
\hline & & $50+$ & 7 & 21 \\
\hline & \multirow{2}{*}{ Security and Fire } & $<50$ & 8 & 0 \\
\hline & & $50+$ & 0 & 77 \\
\hline & \multirow{2}{*}{ Crafts } & $<50$ & 16 & 36 \\
\hline & & $50+$ & 20 & 0 \\
\hline & \multirow{2}{*}{ Line Operators } & $<50$ & 11 & 0 \\
\hline & & $50+$ & 0 & 0 \\
\hline
\end{tabular}

Time Trends

Figure 13. Age-Adjusted Rates for All Diagnoses Combined Among Women and Men from 2003 to 2007*

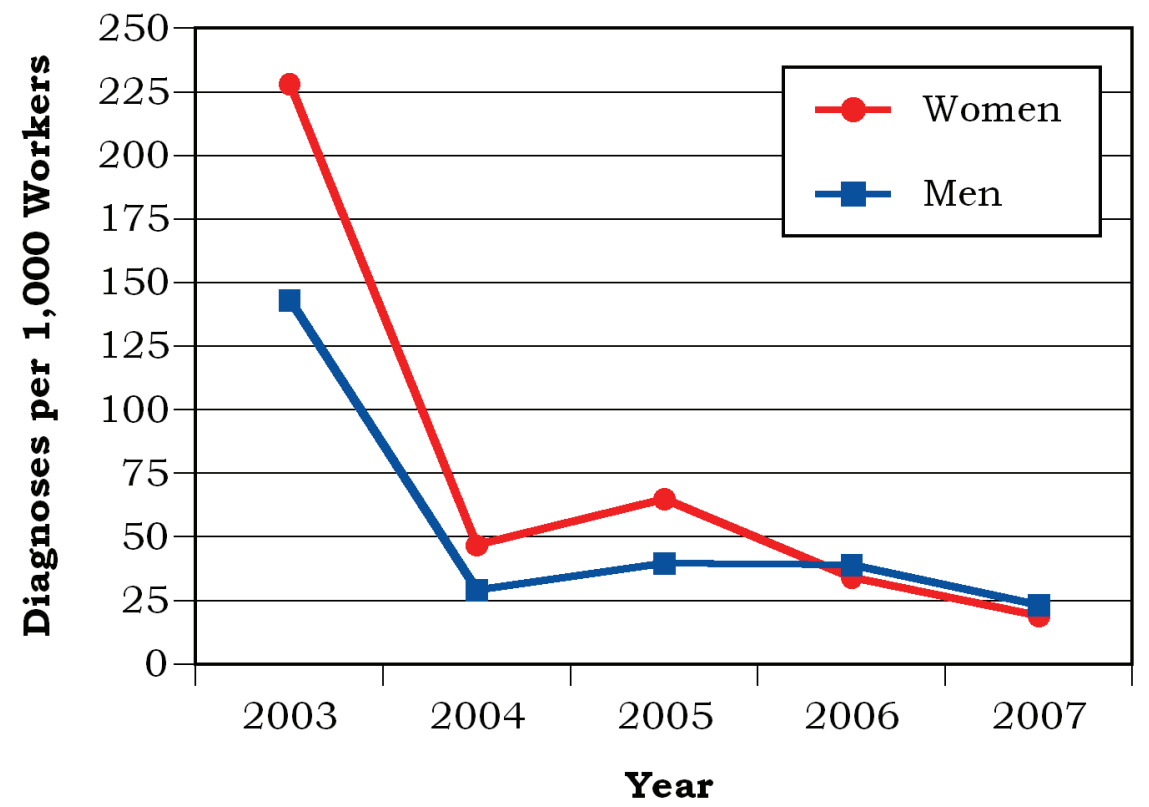

* Standardized to age distribution of 2000 U.S. population. 
Figure 14. Age-Adjusted Rates for Selected Diagnostic

Categories Among Women and Men from 2003 to 2007*

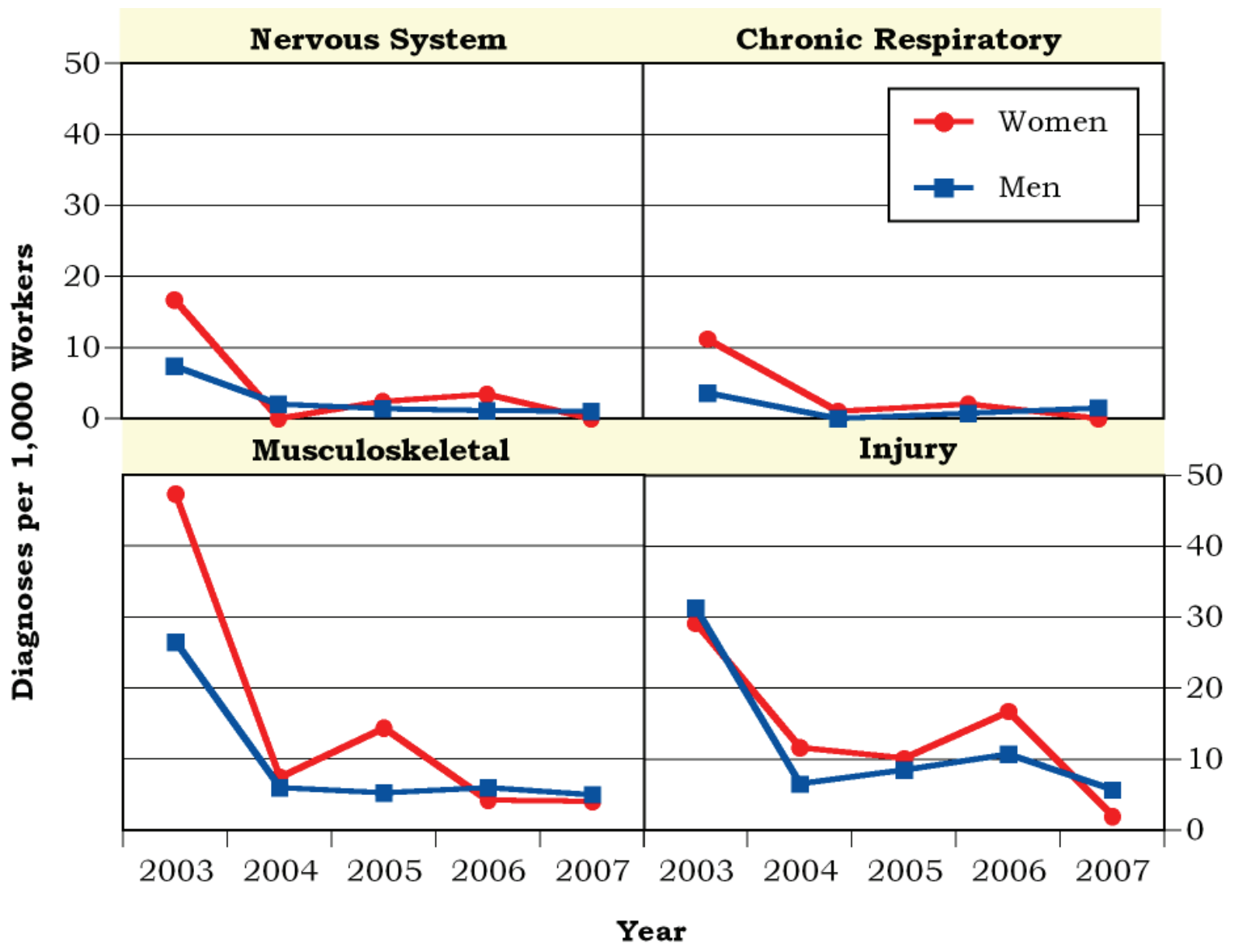

* Standardized to age distribution of 2000 U.S. population. 
Figure 15. Age-Adjusted Rates for All Diagnoses Combined Among Women and Men by Job Category from 2003 to 2007*

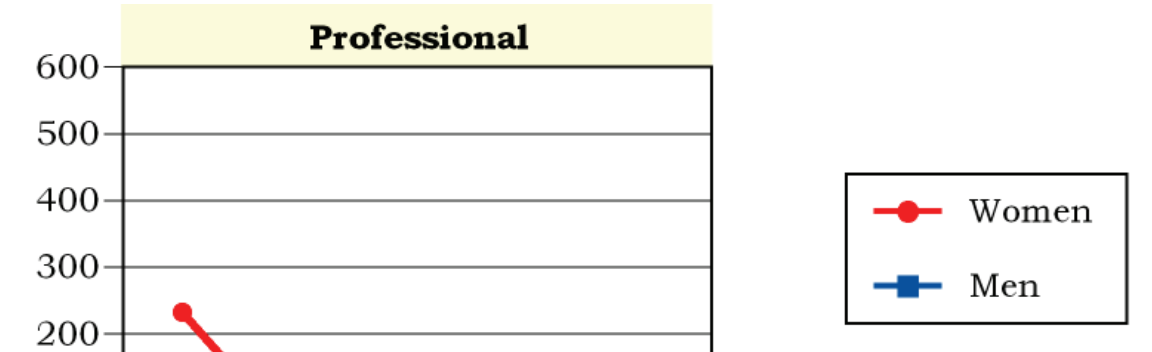

100

0

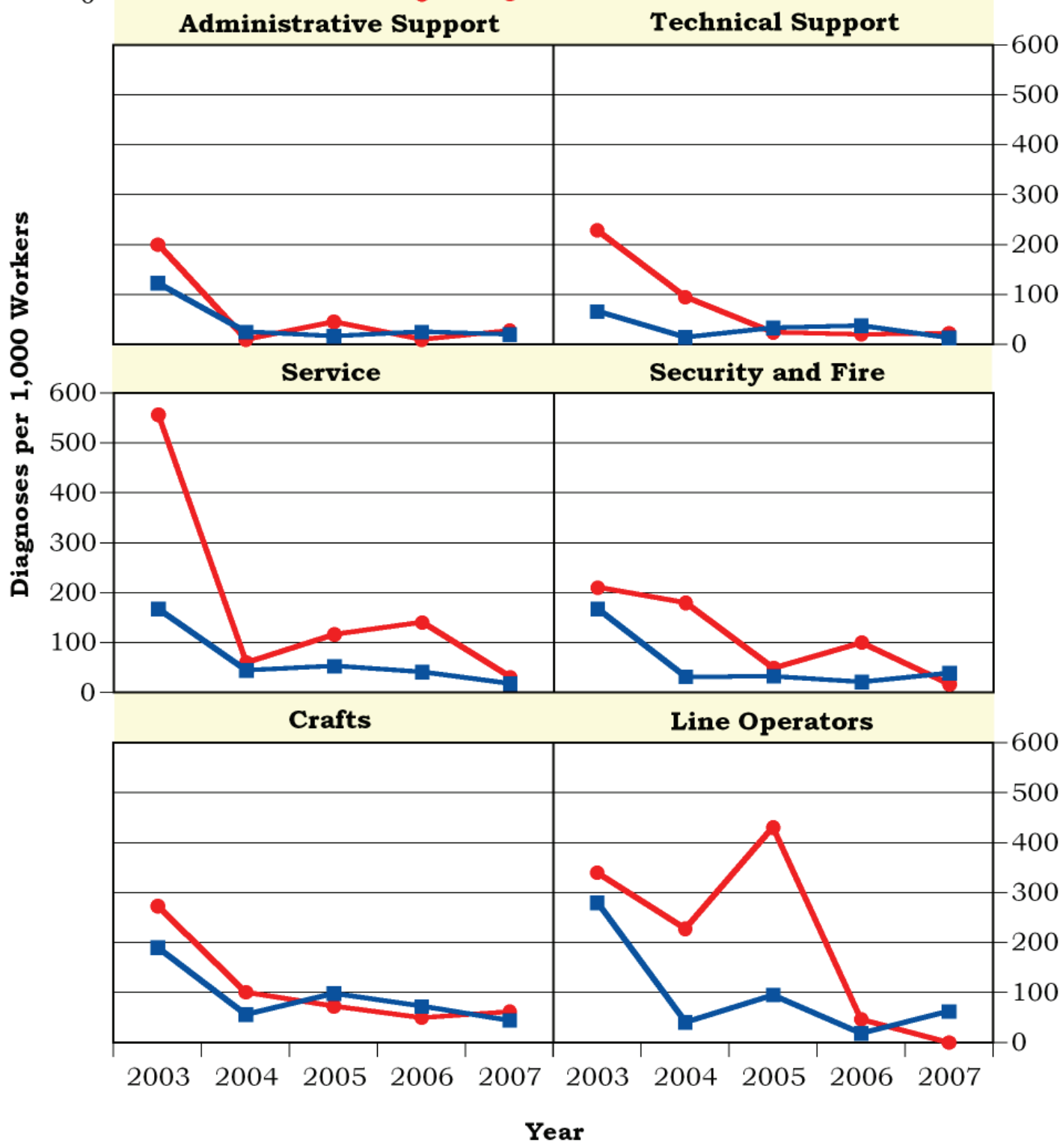

* Standardized to age distribution of 2000 U.S. population. 


\section{Sentinel Health Events for Occupations (SHEOs)}

An occupational sentinel health event (SHEO) is a disease, disability, or death that is likely to be occupationally related. Although sentinel health events may indicate an occupational exposure, many may result from nonoccupational exposures. Sentinel health events are therefore assessed in two categories:

Definite Sentinel Health Events: Diseases that are unlikely to occur in the absence of an occupational exposure (e.g., asbestosis).

Possible Sentinel Health Events: Diseases that may be occupational but can also occur in the absence of an occupational exposure (e.g., lung cancer or carpal tunnel syndrome).

Figure 16. Characteristics of SHEOs by Gender

\begin{tabular}{|l|c|c|c|c|}
\hline \multirow{2}{*}{} & \multicolumn{2}{|c|}{$\begin{array}{c}\text { Total Number of } \\
\text { SHEO Diagnoses }\end{array}$} & \multicolumn{2}{c|}{$\begin{array}{c}\text { Total Number of } \\
\text { Days Absent }\end{array}$} \\
\cline { 2 - 5 } & Men & Women & Men & Women \\
\hline Definite & 0 & 0 & 0 & 0 \\
\hline Possible & 1 & 0 & 53 & 0 \\
\hline Total & 1 & 0 & 53 & 0 \\
\hline
\end{tabular}

Figure 17. SHEO Diagnoses by Gender

\begin{tabular}{|l|c|c|}
\hline \multirow{2}{*}{\multicolumn{1}{|c|}{ Diagnoses }} & \multicolumn{2}{c|}{ Gender } \\
\cline { 2 - 3 } & Women & Men \\
\hline Carpal Tunnel Syndrome & $\mathbf{0}$ & $\mathbf{1}$ \\
\hline Pneumonconiosis (Berylliosis) & $\mathbf{0}$ & $\mathbf{0}$ \\
\hline Musculoskeletal Conditions & $\mathbf{0}$ & $\mathbf{0}$ \\
\hline Injuries & $\mathbf{0}$ & $\mathbf{0}$ \\
\hline Other Conditions & $\mathbf{0}$ & $\mathbf{0}$ \\
\hline
\end{tabular}


Occupational Safety and Health Administration (OSHA)-Recordable Events Figure 18. OSHA-Recordable Events by Gender and Age

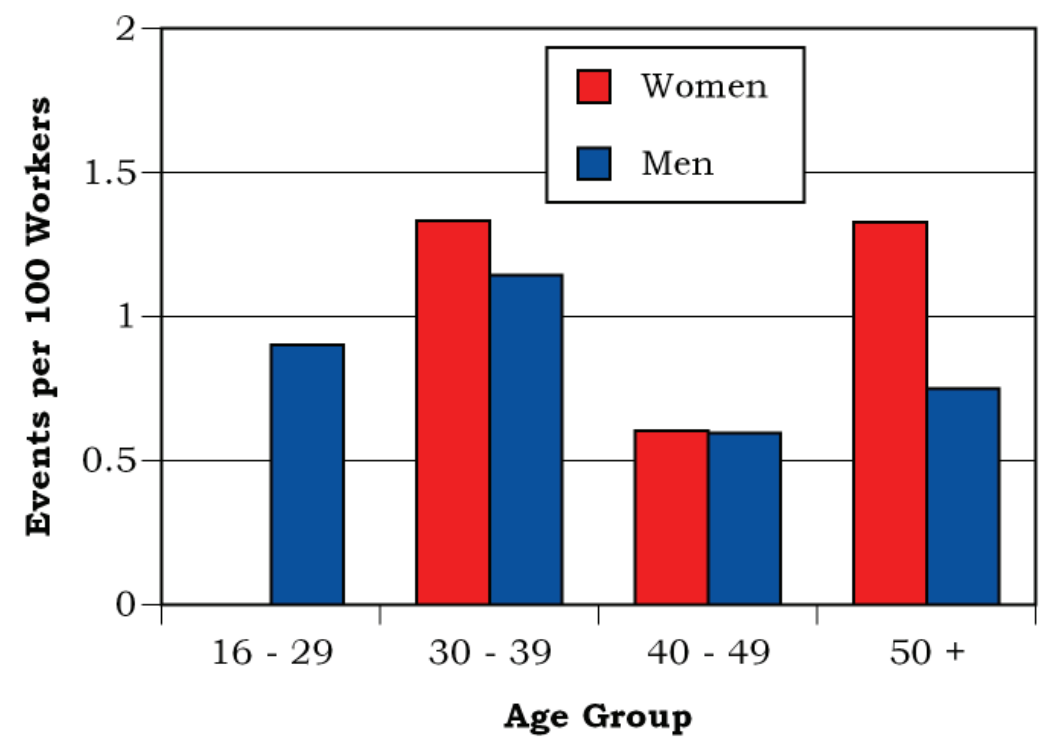

Figure 19. OSHA-Recordable Events by Job Category and Gender

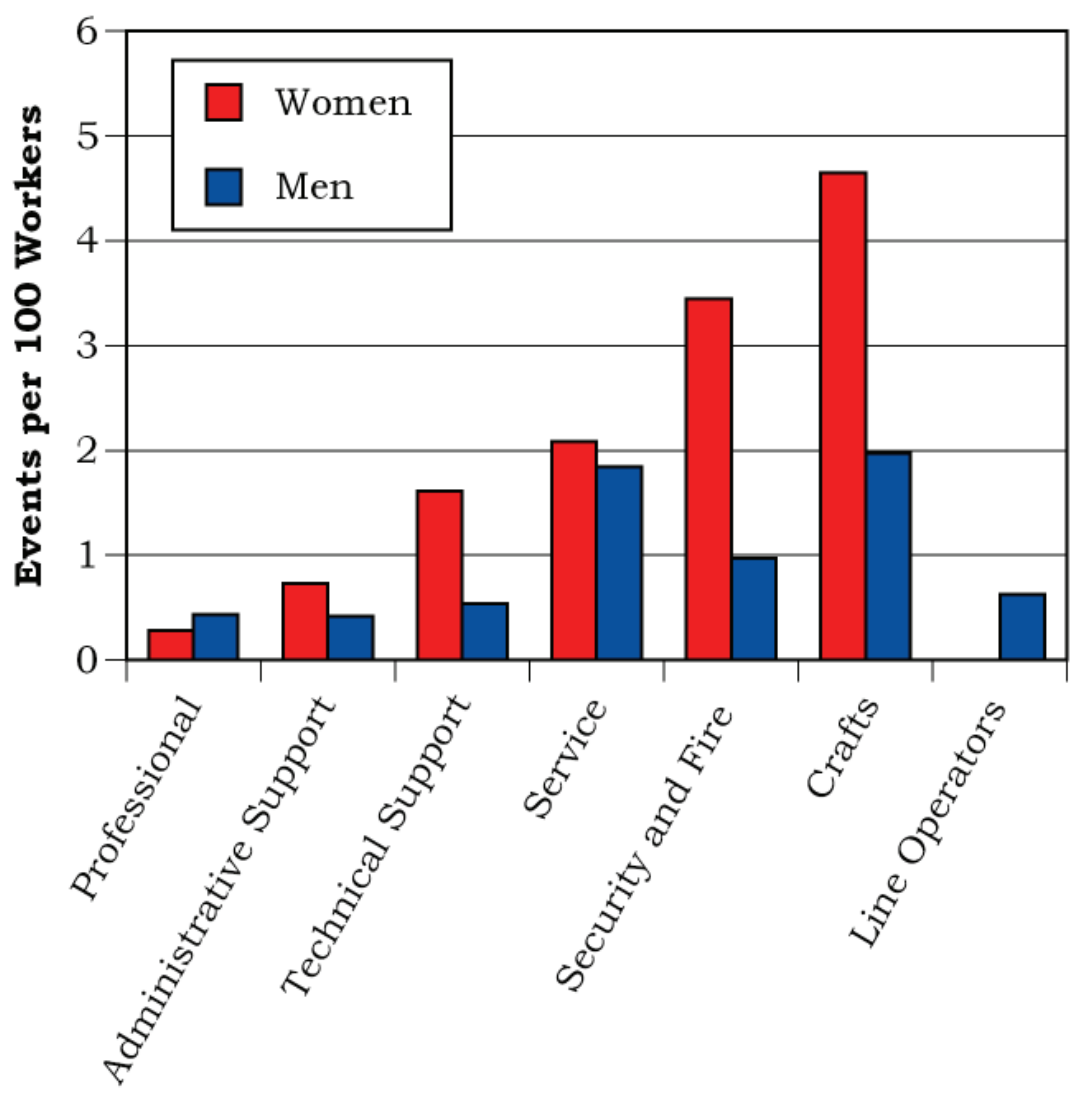

Job Category 


\section{Diagnostic and Accident Categories for OSHA-Recordable Events}

Figure 20. OSHA-Recordable Diagnoses by Diagnostic Category and Gender

\begin{tabular}{|l|c|c|}
\hline \multirow{2}{*}{ Diagnostic Category } & \multicolumn{2}{|c|}{ Gender } \\
\cline { 2 - 3 } & Women & Men \\
\hline Musculoskeletal & $\mathbf{1}$ & $\mathbf{1}$ \\
\hline Nervous System & $\mathbf{0}$ & $\mathbf{2}$ \\
\hline Respiratory & $\mathbf{1}$ & $\mathbf{1}$ \\
\hline Injury & $\mathbf{1 3}$ & $\mathbf{2 8}$ \\
\hline Fractures - Skull & $\mathbf{0}$ & $\mathbf{1}$ \\
\hline Fractures - Upper Limb & $\mathbf{0}$ & $\mathbf{2}$ \\
\hline Fractures - Lower Limb & $\mathbf{0}$ & $\mathbf{3}$ \\
\hline Back Sprains \& Strains & $\mathbf{0}$ & $\mathbf{4}$ \\
\hline Other Sprains \& Strains & $\mathbf{6}$ & $\mathbf{5}$ \\
\hline Open Wounds - Head, Neck, Trunk & $\mathbf{1}$ & $\mathbf{0}$ \\
\hline Open Wounds - Upper Limb & $\mathbf{0}$ & $\mathbf{7}$ \\
\hline Superficial Injuries & $\mathbf{0}$ & $\mathbf{1}$ \\
\hline Bruises & $\mathbf{3}$ & $\mathbf{0}$ \\
\hline Foreign Bodies Entering Orifice & $\mathbf{0}$ & $\mathbf{1}$ \\
\hline Burns & $\mathbf{1}$ & $\mathbf{0}$ \\
\hline Unspecified Injuries & $\mathbf{2}$ & $\mathbf{1}$ \\
\hline Adverse Reactions to Non-Medical & $\mathbf{0}$ & $\mathbf{1}$ \\
\hline Substances & & $\mathbf{2}$ \\
\hline Adverse Reactions to External Causes & $\mathbf{0}$ & \\
\hline
\end{tabular}

Figure 21. OSHA-Recordable Accidents by Type and Gender

\begin{tabular}{|l|c|c|}
\hline \multirow{2}{*}{\multicolumn{1}{|c|}{ Accident Category }} & \multicolumn{2}{|c|}{ Gender } \\
\cline { 2 - 3 } & Women & Men \\
\cline { 2 - 3 } & $\begin{array}{c}\text { Number of } \\
\text { Accidents }\end{array}$ & $\begin{array}{c}\text { Number of } \\
\text { Accidents }\end{array}$ \\
\hline Poisoning - Non-Medicinal & $\mathbf{0}$ & $\mathbf{1}$ \\
\hline Falls & $\mathbf{1}$ & $\mathbf{1}$ \\
\hline Submersion/Suffocation/Foreign Bodies & $\mathbf{0}$ & $\mathbf{1}$ \\
\hline Other Accidents & $\mathbf{2}$ & $\mathbf{8}$ \\
\hline Struck by an Object & $\mathbf{0}$ & $\mathbf{2}$ \\
\hline Machinery & $\mathbf{0}$ & $\mathbf{1}$ \\
\hline Cutting/Piercing Instrument/Object & $\mathbf{0}$ & $\mathbf{2}$ \\
\hline Electric Current & $\mathbf{0}$ & $\mathbf{2}$ \\
\hline Repetitive Trauma & $\mathbf{2}$ & $\mathbf{1}$ \\
\hline Total & $\mathbf{3}$ & $\mathbf{1 1}$ \\
\hline
\end{tabular}




\section{Rates of OSHA-Recordable Events}

Figure 22. OSHA-Recordable Rates by Age and Job Categories Among Women, All Diagnoses Combined

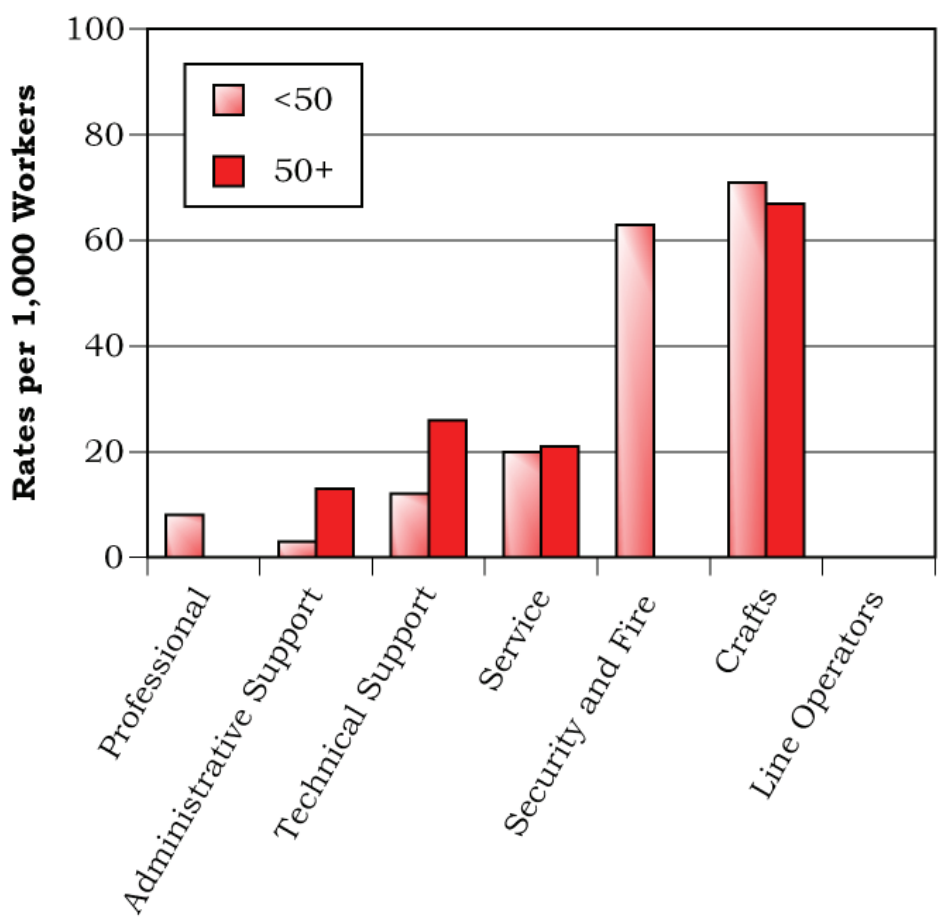

Job Category

Figure 23. OSHA-Recordable Rates by Age and Job Categories Among Men, All Diagnoses Combined

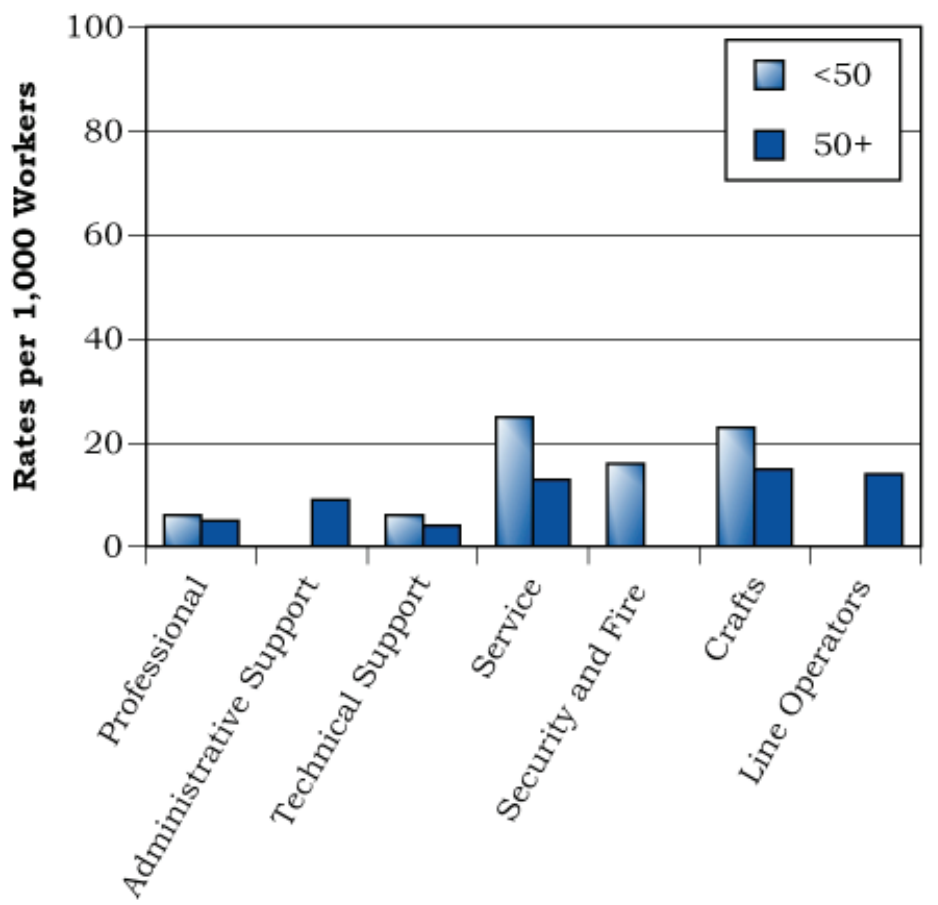

Job Category 


\section{Time Trends for OSHA-Recordable Events}

Figure 24. Age-Adjusted Rates for All OSHA-Recordable Diagnoses Combined Among Women and Men by Job Category from 2003 to 2007*

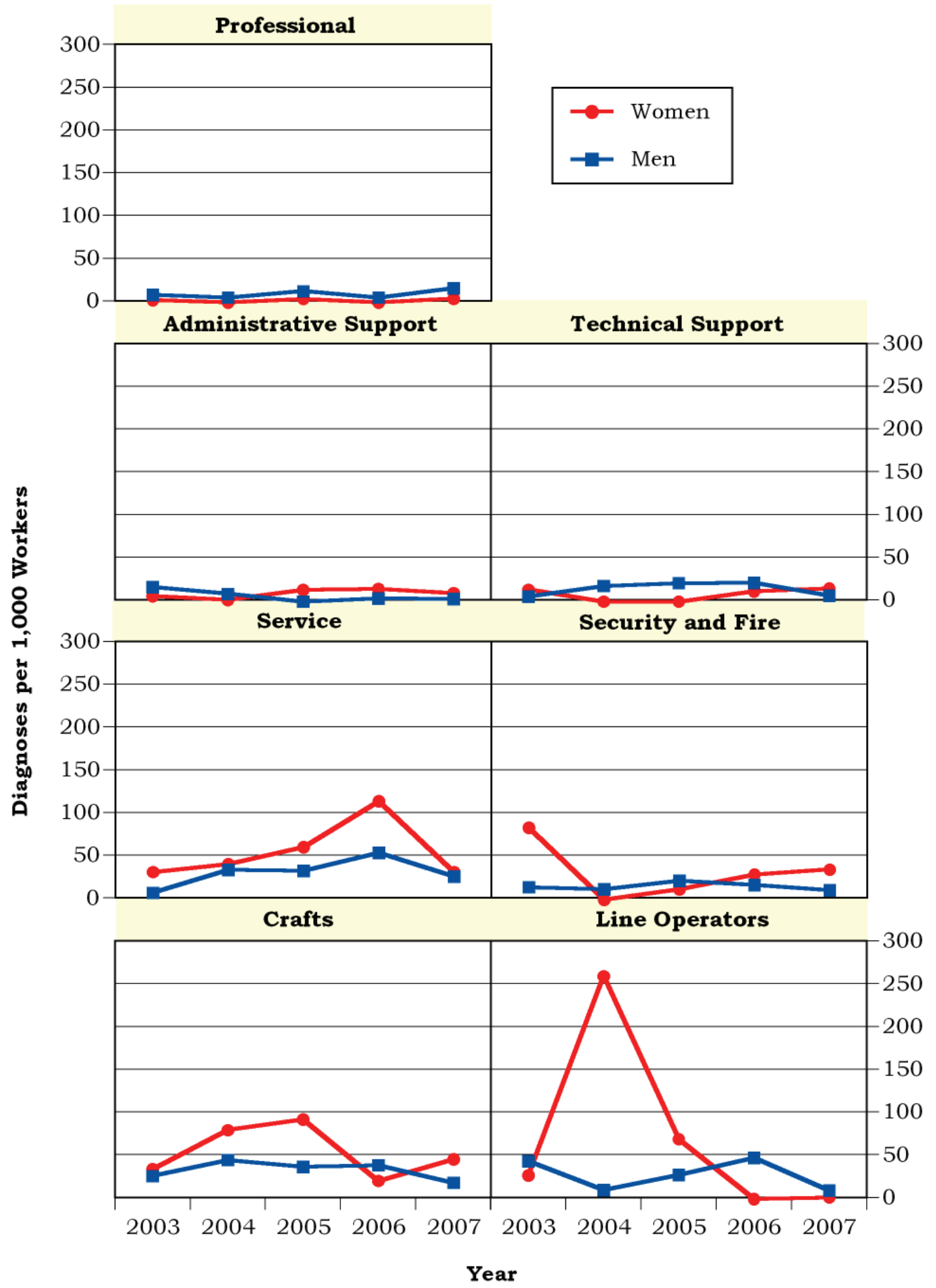

* Standardized to age distribution of 2000 U.S. population. 


\section{Appendices}


Idaho National Laboratory 2007

Absence Data

Appendix A. Work Force by Gender, Age, and Job Category

\begin{tabular}{|c|c|c|c|c|c|c|c|c|c|c|c|}
\hline \multirow{3}{*}{ Job Category } & \multicolumn{5}{|c|}{ Women } & \multicolumn{5}{|c|}{ Men } & \multirow[b]{3}{*}{ TOTAL } \\
\hline & \multicolumn{4}{|c|}{ Age Group } & \multirow[b]{2}{*}{ TOTAL } & \multicolumn{4}{|c|}{ Age Group } & \multirow[b]{2}{*}{ TOTAL } & \\
\hline & $16-29$ & $30-39$ & $40-49$ & $50+$ & & $16-29$ & $30-39$ & $40-49$ & $50+$ & & \\
\hline Professional & 11 & 54 & 183 & 111 & 359 & 50 & 179 & 581 & 788 & 1,598 & 1,957 \\
\hline Administrative Support & 53 & 112 & 217 & 299 & 681 & 37 & 94 & 235 & 346 & 712 & 1,393 \\
\hline Technical Support & 13 & 28 & 45 & 38 & 124 & 40 & 85 & 194 & 235 & 554 & 678 \\
\hline Service & 8 & 14 & 27 & 47 & 96 & 21 & 42 & 58 & 150 & 271 & 367 \\
\hline Security and Fire & 3 & 6 & 7 & 13 & 29 & 12 & 40 & 74 & 80 & 206 & 235 \\
\hline Crafts & 8 & 6 & 14 & 15 & 43 & 52 & 61 & 144 & 199 & 456 & 499 \\
\hline Line Operators & 3 & 5 & 5 & 4 & 17 & 10 & 23 & 55 & 71 & 159 & 176 \\
\hline TOTAL & 99 & 225 & 498 & 527 & 1,349 & 222 & 524 & 1,341 & 1,869 & 3,956 & 5,305 \\
\hline
\end{tabular}

Appendix B. Age Distribution of the Work Force by Gender

\begin{tabular}{|l|r|r|r|r|r|r|r|r|}
\hline \multirow{3}{*}{ Year } & \multicolumn{9}{|c|}{ Women } & \multicolumn{4}{|c|}{ Men } \\
\cline { 2 - 9 } & \multicolumn{1}{|c|}{ Percent Distribution by Age Group } & \multicolumn{1}{c|}{ Percent Distribution by Age Group } \\
\cline { 2 - 9 } & $\mathbf{1 6}-\mathbf{2 9}$ & $\mathbf{3 0}-\mathbf{3 9}$ & $\mathbf{4 0}-\mathbf{4 9}$ & $\mathbf{5 0}+$ & $\mathbf{1 6}-\mathbf{2 9}$ & $\mathbf{3 0}-\mathbf{3 9}$ & $\mathbf{4 0}-\mathbf{4 9}$ & $\mathbf{5 0}+$ \\
\hline $\mathbf{1 9 9 3}$ & 21.01 & 34.35 & 28.99 & 15.65 & 10.30 & 33.87 & 32.67 & 23.17 \\
\hline $\mathbf{1 9 9 4}$ & 22.68 & 33.47 & 27.52 & 16.33 & 10.03 & 30.36 & 32.65 & 26.95 \\
\hline $\mathbf{1 9 9 5}$ & 19.44 & 32.92 & 29.28 & 18.35 & 8.13 & 29.02 & 33.91 & 28.93 \\
\hline $\mathbf{1 9 9 6}$ & 12.46 & 34.28 & 35.33 & 17.93 & 5.75 & 30.58 & 39.53 & 24.14 \\
\hline $\mathbf{1 9 9 7}$ & 10.77 & 32.93 & 36.22 & 20.08 & 5.50 & 27.88 & 39.78 & 26.84 \\
\hline $\mathbf{1 9 9 8}$ & 10.47 & 30.61 & 36.61 & 22.32 & 5.07 & 25.65 & 39.39 & 29.89 \\
\hline $\mathbf{1 9 9 9}$ & 11.02 & 29.59 & 34.58 & 24.81 & 5.68 & 23.14 & 38.01 & 33.18 \\
\hline $\mathbf{2 0 0 0}$ & 10.85 & 28.08 & 35.78 & 25.29 & 5.95 & 22.02 & 37.76 & 34.27 \\
\hline $\mathbf{2 0 0 1}$ & 9.92 & 24.69 & 37.88 & 27.50 & 5.04 & 20.00 & 37.79 & 37.17 \\
\hline $\mathbf{2 0 0 2}$ & 9.18 & 23.21 & 38.52 & 29.10 & 5.57 & 17.85 & 37.32 & 39.27 \\
\hline $\mathbf{2 0 0 3}$ & 7.56 & 21.86 & 40.15 & 30.43 & 6.98 & 16.49 & 38.49 & 38.05 \\
\hline $\mathbf{2 0 0 4}$ & 7.96 & 19.24 & 39.03 & 33.77 & 7.19 & 15.16 & 36.15 & 41.50 \\
\hline $\mathbf{2 0 0 5}$ & 10.55 & 17.03 & 38.37 & 34.05 & 11.18 & 16.18 & 33.05 & 39.58 \\
\hline $\mathbf{2 0 0 6}$ & 8.17 & 16.09 & 37.81 & 37.93 & 7.88 & 14.65 & 33.19 & 44.28 \\
\hline $\mathbf{2 0 0 7}$ & 7.34 & 16.68 & 36.92 & 39.07 & 5.61 & 13.25 & 33.90 & 47.24 \\
\hline
\end{tabular}


Idaho National Laboratory 2007

Absence Data

Appendix C. Total Number of Workers Who Reported at Least One Absence by Gender, Age, and Job Category*

\begin{tabular}{|c|c|c|c|c|c|c|c|c|c|c|c|}
\hline \multirow{3}{*}{ Job Category } & \multicolumn{5}{|c|}{ Women } & \multicolumn{5}{|c|}{ Men } & \multirow[b]{3}{*}{ TOTAL } \\
\hline & \multicolumn{4}{|c|}{ Age Group } & \multirow[b]{2}{*}{ TOTAL } & \multicolumn{4}{|c|}{ Age Group } & \multirow[b]{2}{*}{ TOTAL } & \\
\hline & $16-29$ & 30 - 39 & $40-49$ & $50+$ & & $16-29$ & 30 - 39 & $40-49$ & $50+$ & & \\
\hline Professional & 0 & 0 & 2 & 1 & 3 & 0 & 3 & 4 & 19 & 26 & 29 \\
\hline Administrative Support & 2 & 1 & 2 & 4 & 9 & 0 & 1 & 3 & 13 & 17 & 26 \\
\hline Technical Support & 0 & 1 & 0 & 1 & 2 & 0 & 0 & 5 & 4 & 9 & 11 \\
\hline Service & 0 & 0 & 1 & 2 & 3 & 0 & 0 & 2 & 7 & 9 & 12 \\
\hline Security and Fire & 0 & 0 & 0 & 1 & 1 & 0 & 1 & 2 & 5 & 8 & 9 \\
\hline Crafts & 0 & 0 & 2 & 1 & 3 & 0 & 0 & 13 & 13 & 26 & 29 \\
\hline Line Operators & 0 & 0 & 0 & 0 & 0 & 1 & 0 & 2 & 5 & 8 & 8 \\
\hline TOTAL & 2 & 2 & 7 & 10 & 21 & 1 & 5 & 31 & 66 & 103 & 124 \\
\hline
\end{tabular}

*Only those job categories and gender/age combinations with at least one absence appear in this table.

Appendix D. Total Number of Absences by Gender, Age, and Job Category*

\begin{tabular}{|c|c|c|c|c|c|c|c|c|c|c|c|}
\hline \multirow{3}{*}{ Job Category } & \multicolumn{5}{|c|}{ Women } & \multicolumn{5}{|c|}{ Men } & \multirow[b]{3}{*}{ TOTAL } \\
\hline & \multicolumn{4}{|c|}{ Age Group } & \multirow[b]{2}{*}{ TOTAL } & \multicolumn{4}{|c|}{ Age Group } & \multirow[b]{2}{*}{ TOTAL } & \\
\hline & $16-29$ & 30 - 39 & $40-49$ & $50+$ & & $16-29$ & 30 - 39 & $40-49$ & $50+$ & & \\
\hline Professional & 0 & 0 & 2 & 1 & 3 & 0 & 3 & 4 & 20 & 27 & 30 \\
\hline Administrative Support & 2 & 1 & 2 & 4 & 9 & 0 & 1 & 3 & 14 & 18 & 27 \\
\hline Technical Support & 0 & 1 & 0 & 1 & 2 & 0 & 0 & 5 & 5 & 10 & 12 \\
\hline Service & 0 & 0 & 1 & 2 & 3 & 0 & 0 & 2 & 7 & 9 & 12 \\
\hline Security and Fire & 0 & 0 & 0 & 1 & 1 & 0 & 1 & 2 & 6 & 9 & 10 \\
\hline Crafts & 0 & 0 & 2 & 1 & 3 & 0 & 0 & 13 & 14 & 27 & 30 \\
\hline Line Operators & 0 & $\overline{0}$ & 0 & 0 & 0 & 1 & 0 & 2 & 5 & 8 & 8 \\
\hline TOTAL & 2 & 2 & 7 & 10 & 21 & 1 & 5 & 31 & 71 & 108 & 129 \\
\hline
\end{tabular}

*Only those job categories and gender/age combinations with at least one absence appear in this table. 
Idaho National Laboratory 2007

Absence Data

Appendix E. Distribution of the Number of Calendar Days Missed per Absence by Gender and Age*

\begin{tabular}{|c|c|c|c|c|c|c|c|c|c|c|c|}
\hline \multirow{3}{*}{$\begin{array}{c}\text { Number of } \\
\text { Calendar Days }\end{array}$} & \multicolumn{5}{|c|}{ Women } & \multicolumn{5}{|c|}{ Men } & \multirow[b]{3}{*}{ TOTAL } \\
\hline & \multicolumn{4}{|c|}{ Age Group } & \multirow[b]{2}{*}{ TOTAL } & \multicolumn{4}{|c|}{ Age Group } & \multirow[b]{2}{*}{ TOTAL } & \\
\hline & $16-29$ & $30-39$ & $40-49$ & $50+$ & & $16-29$ & 30 - 39 & $40-49$ & $50+$ & & \\
\hline$<15$ & 2 & 1 & 3 & 1 & 7 & 0 & 1 & 3 & 16 & 20 & 27 \\
\hline $15-28$ & 0 & 0 & 3 & 5 & 8 & 1 & 3 & 7 & 24 & 35 & 43 \\
\hline $29-42$ & 0 & 0 & 1 & 2 & 3 & 0 & 0 & 8 & 14 & 22 & 25 \\
\hline $43-56$ & 0 & 1 & 0 & 0 & 1 & 0 & 0 & 6 & 6 & 12 & 13 \\
\hline $57-91$ & 0 & 0 & 0 & 2 & 2 & 0 & 1 & 5 & 4 & 10 & 12 \\
\hline $92-182$ & 0 & 0 & 0 & 0 & 0 & 0 & 0 & 2 & 7 & 9 & 9 \\
\hline TOTAL & 2 & 2 & 7 & 10 & 21 & 1 & 5 & 31 & 71 & 108 & 129 \\
\hline
\end{tabular}

*Only those gender/age combinations with at least one absence appear in this table. 
Idaho National Laboratory 2007

Absence Data

Appendix F. Distribution of the Number of Calendar Days Missed per Absence by Gender and Job Category*

Women

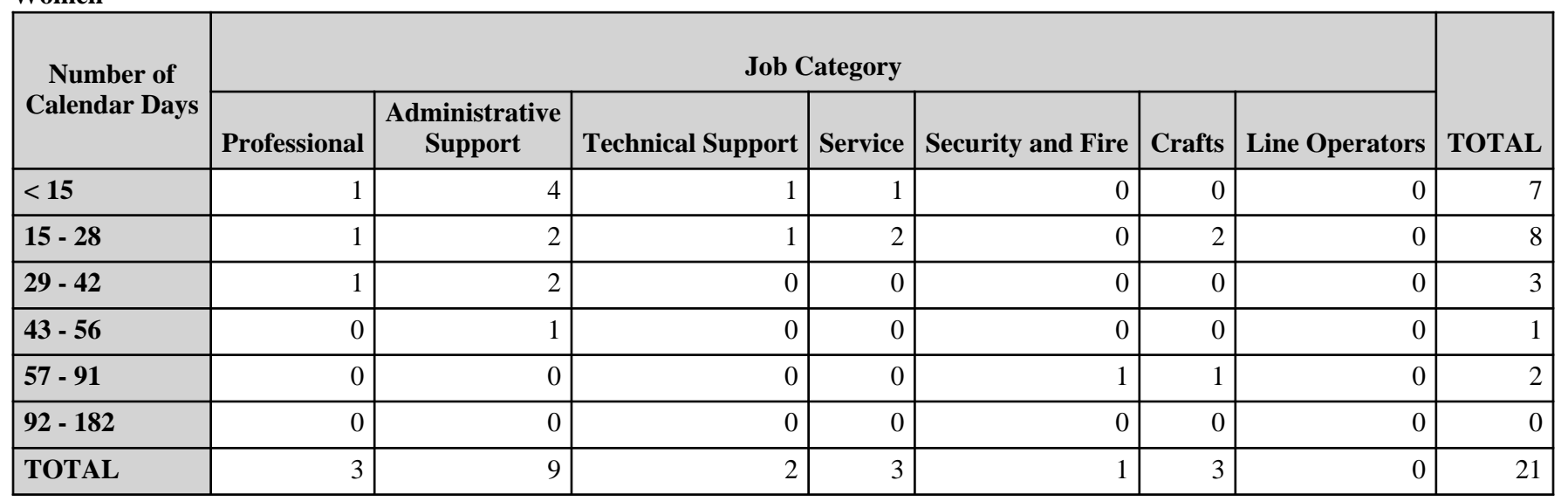

Men

\begin{tabular}{|c|c|c|c|c|c|c|c|c|}
\hline \multirow{2}{*}{$\begin{array}{c}\text { Number of } \\
\text { Calendar Days }\end{array}$} & \multicolumn{7}{|c|}{ Job Category } & \multirow[b]{2}{*}{ TOTAL } \\
\hline & Professional & $\begin{array}{c}\text { Administrative } \\
\text { Support }\end{array}$ & Technical Support & Service & Security and Fire & Crafts & Line Operators & \\
\hline$<15$ & 8 & 2 & 0 & 2 & 5 & 3 & 0 & 20 \\
\hline $15-28$ & 12 & 4 & 5 & 3 & 3 & 5 & 3 & 35 \\
\hline $43-56$ & 2 & 4 & 0 & 1 & 0 & 4 & 1 & 12 \\
\hline $57-91$ & 0 & 4 & 1 & 0 & 0 & 4 & 1 & 10 \\
\hline $92-182$ & 1 & 2 & 1 & 2 & 0 & 2 & 1 & 9 \\
\hline TOTAL & 27 & 18 & 10 & 9 & 9 & 27 & 8 & 108 \\
\hline
\end{tabular}

*Only those gender/job category combinations with at least one absence appear in this table. 
Idaho National Laboratory 2007

Absence Data

Appendix G. Number of Diagnoses in Each Diagnostic Category by Gender and Age*

\begin{tabular}{|c|c|c|c|c|c|c|}
\hline & & \multicolumn{5}{|c|}{ Women } \\
\hline & & \multicolumn{4}{|c|}{ Age Group } & \multirow[b]{2}{*}{ TOTAL } \\
\hline & & $16-29$ & $30-39$ & $40-49$ & $50+$ & \\
\hline Diagnostic Category & ICD-9-CM Code & \multirow[b]{2}{*}{0} & \multirow[b]{2}{*}{0} & \multirow[b]{2}{*}{0} & \multirow[b]{2}{*}{0} & \multirow[b]{2}{*}{0} \\
\hline INFECTIOUS \& PARASITIC DISEASES (DIS) & \begin{tabular}{|c|}
$001-139$ \\
\end{tabular} & & & & & \\
\hline -Intestinal Infectious Dis & 001-009 & 0 & 0 & 0 & 0 & 0 \\
\hline -Polio \& Other Viral CNS Dis & 045-049 & 0 & 0 & 0 & 0 & 0 \\
\hline MALIGNANT NEOPLASMS & $140-208,230-234$ & 0 & 0 & 0 & 1 & 1 \\
\hline -Breast & 174-175 & 0 & 0 & 0 & 1 & 1 \\
\hline -Genitourinary & 179-189 & 0 & 0 & 0 & 0 & 0 \\
\hline -Other \& Unspecified Sites & $190,193-199$ & 0 & 0 & 0 & 0 & 0 \\
\hline -Lymphatic \& Hematopoietic & $200-208$ & 0 & 0 & 0 & 0 & 0 \\
\hline BENIGN \& UNCERTAIN NEOPLASMS & 210-229, 235-239 & 0 & 0 & 0 & 0 & 0 \\
\hline ENDOCRINE/METABOLIC/IMMUNITY & $240-279$ & 0 & 0 & 0 & 0 & 0 \\
\hline -Thyroid Gland Disorders & $240-246$ & 0 & 0 & 0 & 0 & 0 \\
\hline -Other Metabolic \& Immunity Disorders & $270-279$ & 0 & 0 & 0 & 0 & 0 \\
\hline MENTAL DISORDERS & $290-319$ & 0 & 0 & 0 & 0 & 0 \\
\hline -Psychoses & 290-299 & 0 & 0 & 0 & 0 & 0 \\
\hline -Non-Psychotic Disorders & $300-302,306-316$ & 0 & 0 & 0 & 0 & 0 \\
\hline NERVOUS SYSTEM (NS) \& SENSE ORGANS & 320-389 & 0 & 0 & 0 & 1 & 1 \\
\hline -Disorders of Peripheral NS & $350-359$ & 0 & 0 & 0 & 0 & 0 \\
\hline -Disorders of Eye & $360-379$ & 0 & 0 & 0 & 1 & 1 \\
\hline -Dis of Ear \& Mastoid & $380-389$ & 0 & 0 & 0 & 0 & 0 \\
\hline CIRCULATORY SYSTEM & $390-459$ & 0 & 1 & 0 & 0 & 1 \\
\hline -Ischemic Heart Dis & $410-414$ & 0 & 0 & 0 & 0 & 0 \\
\hline -Dis of Pulmonary Circulation & $415-417$ & 0 & 0 & 0 & 0 & 0 \\
\hline -Other Heart Dis & $420-429$ & 0 & 0 & 0 & 0 & 0 \\
\hline -Cerebrovascular Dis & $430-438$ & 0 & 1 & 0 & 0 & 1 \\
\hline -Dis of Arteries \& Capillaries & $440-448$ & 0 & 0 & 0 & 0 & 0 \\
\hline -Dis of Veins, Lymphatics, Other & $451-459$ & 0 & 0 & 0 & 0 & 0 \\
\hline RESPIRATORY SYSTEM & $460-519$ & 3 & 0 & 3 & 2 & 8 \\
\hline -Acute Respiratory Infections & $460-466$ & 3 & 0 & 1 & 0 & 4 \\
\hline -Other Dis Upper Respiratory Tract & $470-478$ & 0 & 0 & 1 & 1 & 2 \\
\hline -Pneumonia \& Influenza & $480-487$ & 0 & 0 & 1 & 1 & 2 \\
\hline -Other Respiratory Dis & $510-519$ & 0 & 0 & 0 & 0 & 0 \\
\hline DIGESTIVE SYSTEM & $520-579$ & 0 & 2 & 3 & 0 & 5 \\
\hline -Esophagus, Stomach, Duodenum & 530-537 & 0 & 2 & 0 & 0 & 2 \\
\hline -Appendicitis & $540-543$ & 0 & 0 & 0 & 0 & 0 \\
\hline -Hernia & $550-553$ & 0 & 0 & 0 & 0 & 0 \\
\hline -Enteritis, Colitis & $555-558$ & 0 & 0 & 1 & 0 & 1 \\
\hline -Other Intestinal Dis & $560-569$ & 0 & 0 & 0 & 0 & 0 \\
\hline
\end{tabular}

(Continued)

*Only those diagnostic categories and gender/age combinations with at least one occurrence appear in this table. 
Idaho National Laboratory 2007

Absence Data

Appendix G. Number of Diagnoses in Each Diagnostic Category by Gender and Age*

\begin{tabular}{|c|c|c|c|c|c|c|c|}
\hline & & \multicolumn{5}{|c|}{ Men } & \multirow[b]{3}{*}{ TOTAL } \\
\hline & & \multicolumn{4}{|c|}{ Age Group } & \multirow[b]{2}{*}{ TOTAL } & \\
\hline & & $16-29$ & 30 - 39 & $40-49$ & $\mathbf{5 0 +}$ & & \\
\hline INFECTIOUS \& PARASITIC DISEASES (DIS) & \begin{tabular}{|c|}
$001-139$ \\
\end{tabular} & 0 & 0 & 1 & 1 & 2 & 2 \\
\hline -Intestinal Infectious Dis & 001-009 & 0 & 0 & 1 & 0 & 1 & 1 \\
\hline MALIGNANT NEOPLASMS & $140-208,230-234$ & 0 & 0 & 1 & 3 & 4 & 5 \\
\hline -Breast & 174-175 & 0 & 0 & 0 & 0 & 0 & 1 \\
\hline -Genitourinary & 179-189 & 0 & 0 & 0 & 2 & 2 & 2 \\
\hline -Other \& Unspecified Sites & $190,193-199$ & 0 & 0 & 1 & 0 & 1 & 1 \\
\hline -Thyroid Gland Disorders & $240-246$ & 0 & 0 & 0 & 1 & 1 & 1 \\
\hline -Other Metabolic \& Immunity Disorders & $270-279$ & 0 & 0 & 1 & 0 & 1 & 1 \\
\hline MENTAL DISORDERS & $290-319$ & 0 & 0 & 3 & 3 & 6 & 6 \\
\hline -Psychoses & $290-299$ & 0 & 0 & 2 & 0 & 2 & 2 \\
\hline -Non-Psychotic Disorders & $300-302,306-316$ & 0 & 0 & 1 & 3 & 4 & 4 \\
\hline NERVOUS SYSTEM (NS) \& SENSE ORGANS & $320-389$ & 0 & 0 & 0 & 5 & 5 & 6 \\
\hline -Disorders of Peripheral NS & $350-359$ & 0 & 0 & 0 & 2 & 2 & 2 \\
\hline -Disorders of Eye & $360-379$ & 0 & 0 & 0 & 0 & 0 & 1 \\
\hline -Cerebrovascular Dis & $430-438$ & 0 & 0 & 0 & 0 & 0 & 1 \\
\hline -Dis of Arteries \& Capillaries & $440-448$ & 0 & 0 & 0 & 1 & 1 & 1 \\
\hline -Dis of Veins, Lymphatics, Other & 451-459 & 0 & 0 & 0 & 4 & 4 & 4 \\
\hline RESPIRATORY SYSTEM & $460-519$ & 0 & 2 & 6 & 13 & 21 & 29 \\
\hline -Acute Respiratory Infections & $460-466$ & 0 & 1 & 2 & 4 & 7 & 11 \\
\hline -Other Dis Upper Respiratory Tract & $470-478$ & 0 & 0 & 1 & 3 & 4 & 6 \\
\hline -Pneumonia \& Influenza & $480-487$ & 0 & 1 & 3 & 5 & 9 & 11 \\
\hline -Other Respiratory Dis & $510-519$ & 0 & 0 & 0 & 1 & 1 & 1 \\
\hline DIGESTIVE SYSTEM & $520-579$ & 0 & 0 & 4 & 13 & 17 & 22 \\
\hline -Esophagus, Stomach, Duodenum & $530-537$ & 0 & 0 & 0 & 1 & 1 & 3 \\
\hline -Appendicitis & $540-543$ & 0 & 0 & 1 & 1 & 2 & 2 \\
\hline -Hernia & $550-553$ & 0 & 0 & 2 & 9 & 11 & 11 \\
\hline -Enteritis, Colitis & $555-558$ & 0 & 0 & 0 & 1 & 1 & 2 \\
\hline -Other Intestinal Dis & $560-569$ & 0 & 0 & 0 & 1 & 1 & 1 \\
\hline
\end{tabular}

(Continued)

*Only those diagnostic categories and gender/age combinations with at least one occurrence appear in this table. 
Idaho National Laboratory 2007

Absence Data

Appendix G. Number of Diagnoses in Each Diagnostic Category by Gender and Age*

\begin{tabular}{|c|c|c|c|c|c|c|}
\hline & & \multicolumn{5}{|c|}{ Women } \\
\hline & & \multicolumn{4}{|c|}{ Age Group } & \multirow[b]{2}{*}{ TOTAL } \\
\hline & & $16-29$ & $30-39$ & $40-49$ & $50+$ & \\
\hline Diagnostic Category & ICD-9-CM Code & \multirow[b]{2}{*}{0} & \multirow[b]{2}{*}{0} & \multirow[b]{2}{*}{2} & \multirow[b]{2}{*}{0} & \multirow[b]{2}{*}{2} \\
\hline -Other Digestive Dis & $570-579$ & & & & & \\
\hline GENITOURINARY SYSTEM & $580-629$ & 0 & 0 & 1 & 1 & 2 \\
\hline -Other Urinary Dis & $590-599$ & 0 & 0 & 0 & 0 & 0 \\
\hline -Male Genital Organ Dis & $600-608$ & 0 & 0 & 0 & 0 & 0 \\
\hline -Breast Disorders & $610-611$ & 0 & 0 & 0 & 1 & 1 \\
\hline -Other Female Disorders & $617-629$ & 0 & 0 & 1 & 0 & 1 \\
\hline SKIN \& SUBCUTANEOUS TISSUE & $680-709$ & 0 & 0 & 0 & 0 & 0 \\
\hline -Infections & $680-686$ & 0 & 0 & 0 & 0 & 0 \\
\hline $\begin{array}{l}\text { MUSCULOSKELETAL \& CONNECTIVE } \\
\text { TISSUE }\end{array}$ & \begin{tabular}{|l|}
$710-739$ \\
\end{tabular} & 0 & 0 & 1 & 2 & 3 \\
\hline -Arthropathies & $710-719$ & 0 & 0 & 1 & 1 & 2 \\
\hline -Dorsopathies & $720-724$ & 0 & 0 & 0 & 0 & 0 \\
\hline -Rheumatism, Excluding Back & $725-729$ & 0 & 0 & 0 & 1 & 1 \\
\hline -Other Dis \& Acquired Deformities & $730-739$ & 0 & 0 & 0 & 0 & 0 \\
\hline $\begin{array}{l}\text { SYMPTOMS, SIGNS, \& ILL-DEFINED } \\
\text { CONDITIONS }\end{array}$ & 780-799 & 0 & 0 & 0 & 0 & 0 \\
\hline -Symptoms & $780-789$ & 0 & 0 & 0 & 0 & 0 \\
\hline -IIl-Defined \& Unknown Causes & 797-799 & 0 & 0 & 0 & 0 & 0 \\
\hline INJURY \& POISONING & \begin{tabular}{|l|}
$800-999$ \\
\end{tabular} & 0 & 0 & 1 & 4 & 5 \\
\hline -Fracture - Upper Limb & 810-819 & 0 & 0 & 0 & 0 & 0 \\
\hline -Fracture - Lower Limb & $820-829$ & 0 & 0 & 0 & 2 & 2 \\
\hline -Dislocation & $830-839$ & 0 & 0 & 0 & 1 & 1 \\
\hline -Sprains \& Strains - Back & 846-847 & 0 & 0 & 0 & 0 & 0 \\
\hline -Sprains \& Strains - Other & $840-845,848$ & 0 & 0 & 1 & 1 & 2 \\
\hline -Open Wound - Lower Limb & $890-897$ & 0 & 0 & 0 & 0 & 0 \\
\hline -Contusion & $920-924$ & 0 & 0 & 0 & 0 & 0 \\
\hline -Complications of Surgical/Medical Care & 996-999 & 0 & 0 & 0 & 0 & 0 \\
\hline $\begin{array}{l}\text { HEALTH STATUS/HEALTH SERVICE } \\
\text { CONTACT }\end{array}$ & V01-V82 & 0 & 0 & 0 & 0 & 0 \\
\hline -Personal \& Family History & V10-V19 & 0 & 0 & 0 & 0 & 0 \\
\hline -Specific Procedures/Aftercare & V50-V59 & 0 & 0 & 0 & 0 & 0 \\
\hline
\end{tabular}

*Only those diagnostic categories and gender/age combinations with at least one occurrence appear in this table. 
Idaho National Laboratory 2007

Absence Data

Appendix G. Number of Diagnoses in Each Diagnostic Category by Gender and Age*

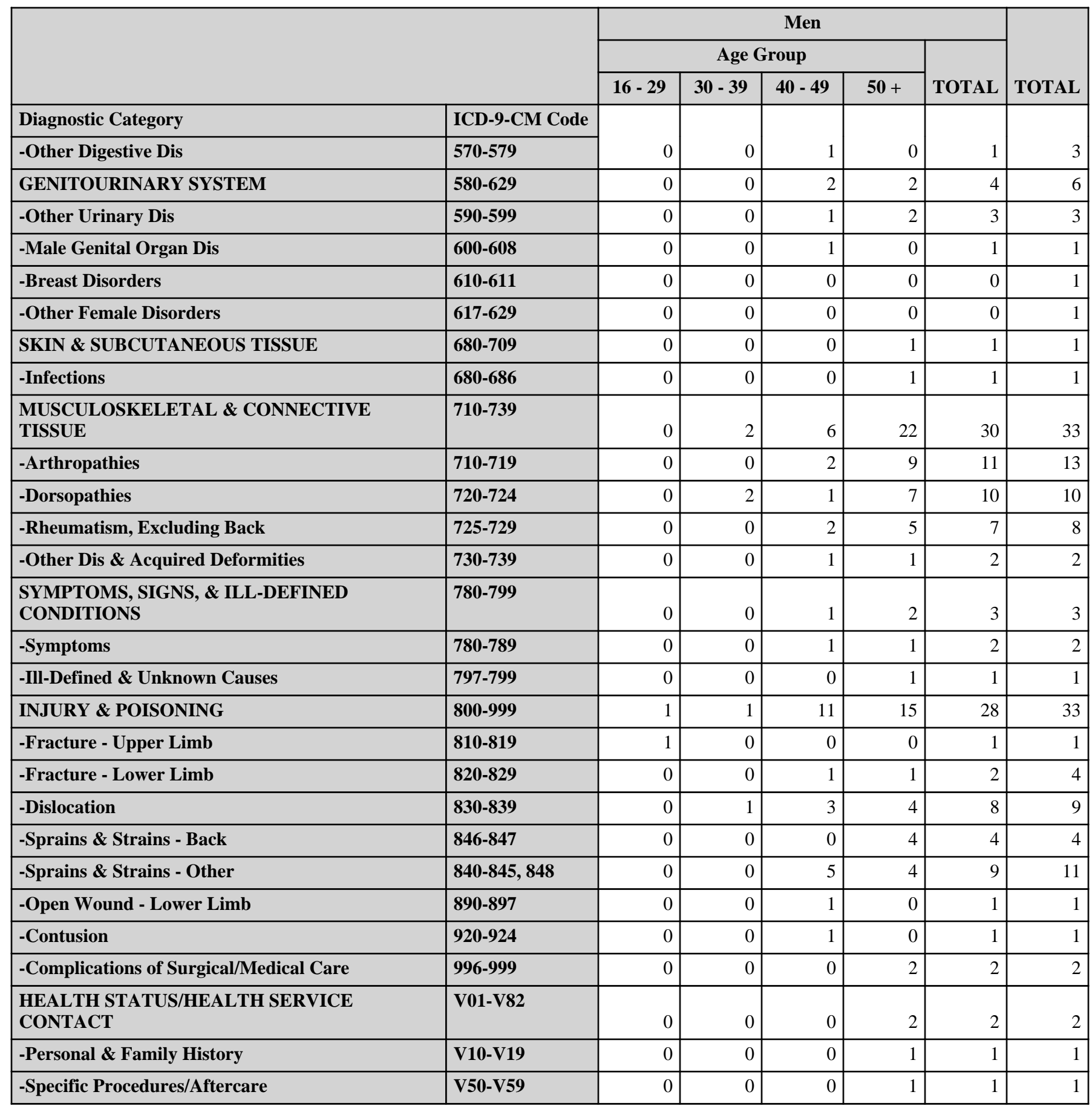

\begin{tabular}{|c|c|c|c|c|c|c|c|c|c|c|c|}
\hline & \multicolumn{5}{|c|}{ Women } & \multicolumn{5}{|c|}{ Men } & \multirow[b]{3}{*}{ TOTAL } \\
\hline & \multicolumn{4}{|c|}{ Age Group } & \multirow[b]{2}{*}{ TOTAL } & \multicolumn{4}{|c|}{ Age Group } & \multirow[b]{2}{*}{ TOTAL } & \\
\hline & $16-29$ & $30-39$ & $40-49$ & $50+$ & & $16-29$ & 30 - 39 & $40-49$ & $50+$ & & \\
\hline Diagnostic Category & \multirow[b]{2}{*}{3} & \multirow[b]{2}{*}{3} & \multirow[b]{2}{*}{9} & \multirow[b]{2}{*}{11} & \multirow[b]{2}{*}{26} & \multirow[b]{2}{*}{1} & \multirow[b]{2}{*}{5} & \multirow[b]{2}{*}{36} & \multirow[b]{2}{*}{98} & \multirow[b]{2}{*}{140} & \multirow[b]{2}{*}{166} \\
\hline TOTAL & & & & & & & & & & & \\
\hline
\end{tabular}

*Only those diagnostic categories and gender/age combinations with at least one occurrence appear in this table. 
Idaho National Laboratory 2007

Absence Data

Appendix H. Total Number of Calendar Days Absent in Each Diagnostic Category by Gender and Age*

\begin{tabular}{|c|c|c|c|c|c|c|}
\hline & & \multicolumn{5}{|c|}{ Women } \\
\hline & & \multicolumn{4}{|c|}{ Age Group } & \multirow[b]{2}{*}{ TOTAL } \\
\hline & & $16-29$ & $30-39$ & $40-49$ & $50+$ & \\
\hline Diagnostic Category & ICD-9-CM Code & \multirow[b]{2}{*}{0} & \multirow[b]{2}{*}{0} & \multirow[b]{2}{*}{0} & \multirow[b]{2}{*}{0} & \multirow[b]{2}{*}{0} \\
\hline INFECTIOUS \& PARASITIC DISEASES (DIS) & 001-139 & & & & & \\
\hline MALIGNANT NEOPLASMS & $140-208,230-234$ & 0 & 0 & 0 & 91 & 91 \\
\hline BENIGN \& UNCERTAIN NEOPLASMS & 210-229, 235-239 & 0 & 0 & 0 & 0 & 0 \\
\hline ENDOCRINE/METABOLIC/IMMUNITY & $240-279$ & 0 & 0 & 0 & 0 & 0 \\
\hline MENTAL DISORDERS & $290-319$ & 0 & 0 & 0 & 0 & 0 \\
\hline NERVOUS SYSTEM (NS) \& SENSE ORGANS & 320-389 & 0 & 0 & 0 & 20 & 20 \\
\hline CIRCULATORY SYSTEM & $390-459$ & 0 & 43 & 0 & 0 & 43 \\
\hline RESPIRATORY SYSTEM & $460-519$ & 21 & 0 & 42 & 29 & 92 \\
\hline DIGESTIVE SYSTEM & $520-579$ & 0 & 13 & 37 & 0 & 50 \\
\hline GENITOURINARY SYSTEM & $580-629$ & 0 & 0 & 11 & 30 & 41 \\
\hline SKIN \& SUBCUTANEOUS TISSUE & 680-709 & 0 & 0 & 0 & 0 & 0 \\
\hline $\begin{array}{l}\text { MUSCULOSKELETAL \& CONNECTIVE } \\
\text { TISSUE }\end{array}$ & \begin{tabular}{|l|}
$710-739$ \\
\end{tabular} & 0 & 0 & 38 & 25 & 63 \\
\hline $\begin{array}{l}\text { SYMPTOMS, SIGNS, \& ILL-DEFINED } \\
\text { CONDITIONS }\end{array}$ & $780-799$ & 0 & 0 & 0 & 0 & 0 \\
\hline INJURY \& POISONING & $800-999$ & 0 & 0 & 19 & 151 & 170 \\
\hline
\end{tabular}

\begin{tabular}{|c|c|c|c|c|c|c|c|}
\hline & & \multicolumn{5}{|c|}{ Men } & \multirow[b]{3}{*}{ TOTAL } \\
\hline & & \multicolumn{4}{|c|}{ Age Group } & \multirow[b]{2}{*}{ TOTAL } & \\
\hline & & $16-29$ & $30-39$ & $40-49$ & $50+$ & & \\
\hline Diagnostic Category & ICD-9-CM Code & & & & & & \\
\hline INFECTIOUS \& PARASITIC DISEASES (DIS) & 001-139 & 0 & 0 & 21 & 24 & 45 & 45 \\
\hline MALIGNANT NEOPLASMS & $140-208,230-234$ & 0 & 0 & 70 & 104 & 174 & 265 \\
\hline BENIGN \& UNCERTAIN NEOPLASMS & $210-229,235-239$ & 0 & 0 & 0 & 26 & 26 & 26 \\
\hline ENDOCRINE/METABOLIC/IMMUNITY & 240-279 & 0 & 0 & 20 & 19 & 39 & 39 \\
\hline MENTAL DISORDERS & 290-319 & 0 & 0 & 75 & 67 & 142 & 142 \\
\hline NERVOUS SYSTEM (NS) \& SENSE ORGANS & 320-389 & 0 & 0 & 0 & 113 & 113 & 133 \\
\hline CIRCULATORY SYSTEM & 390-459 & 0 & 0 & 0 & 738 & 738 & 781 \\
\hline RESPIRATORY SYSTEM & $460-519$ & 0 & 37 & 182 & 221 & 440 & 532 \\
\hline DIGESTIVE SYSTEM & $520-579$ & 0 & 0 & 120 & 416 & 536 & 586 \\
\hline GENITOURINARY SYSTEM & 580-629 & 0 & 0 & 47 & 18 & 65 & 106 \\
\hline SKIN \& SUBCUTANEOUS TISSUE & 680-709 & 0 & 0 & 0 & 33 & 33 & 33 \\
\hline $\begin{array}{l}\text { MUSCULOSKELETAL \& CONNECTIVE } \\
\text { TISSUE }\end{array}$ & 710-739 & 0 & 87 & 330 & 999 & 1,416 & 1,479 \\
\hline $\begin{array}{l}\text { SYMPTOMS, SIGNS, \& ILL-DEFINED } \\
\text { CONDITIONS }\end{array}$ & 780-799 & 0 & 0 & 20 & 176 & 196 & 196 \\
\hline INJURY \& POISONING & $800-999$ & 16 & 11 & 442 & 365 & 834 & 1,004 \\
\hline
\end{tabular}

\footnotetext{
*Absences with >1 ICD-9-CM code in the same diagnostic category were counted only once. Only those diagnostic categories and gender/age combinations with at least one occurrence appear in this table.
} 
Idaho National Laboratory 2007

Absence Data

Appendix I. Number of Diagnoses in Each Diagnostic Category by Gender and Job Category*

\begin{tabular}{|c|c|c|c|c|}
\hline & & \multicolumn{3}{|c|}{ Women } \\
\hline & & \multicolumn{3}{|c|}{ Job Category } \\
\hline & & Professional & $\begin{array}{l}\text { Administrative } \\
\text { Support }\end{array}$ & Technical Support \\
\hline Diagnostic Category & ICD-9-CM Code & \multirow[b]{2}{*}{0} & \multirow[b]{2}{*}{0} & \multirow[b]{2}{*}{0} \\
\hline MALIGNANT NEOPLASMS & $140-208,230-234$ & & & \\
\hline -Breast & 174-175 & 0 & 0 & 0 \\
\hline NERVOUS SYSTEM (NS) \& SENSE ORGANS & $320-389$ & 0 & 0 & 0 \\
\hline -Disorders of Eye & $360-379$ & 0 & 0 & 0 \\
\hline CIRCULATORY SYSTEM & $390-459$ & 0 & 1 & $\overline{0}$ \\
\hline -Cerebrovascular Dis & $430-438$ & 0 & 1 & 0 \\
\hline RESPIRATORY SYSTEM & $460-519$ & 1 & 4 & 1 \\
\hline -Acute Respiratory Infections & $460-466$ & 0 & 3 & 0 \\
\hline -Other Dis Upper Respiratory Tract & $470-478$ & 0 & 0 & 1 \\
\hline -Pneumonia \& Influenza & $480-487$ & 1 & 1 & 0 \\
\hline DIGESTIVE SYSTEM & $520-579$ & 0 & 1 & 2 \\
\hline -Esophagus, Stomach, Duodenum & $530-537$ & 0 & 0 & 2 \\
\hline -Enteritis, Colitis & $555-558$ & 0 & 0 & 0 \\
\hline -Other Digestive Dis & $570-579$ & 0 & 1 & 0 \\
\hline GENITOURINARY SYSTEM & $580-629$ & 1 & 1 & 0 \\
\hline -Breast Disorders & $610-611$ & 0 & 1 & 0 \\
\hline -Other Female Disorders & $617-629$ & 1 & 0 & 0 \\
\hline $\begin{array}{l}\text { MUSCULOSKELETAL \& CONNECTIVE } \\
\text { TISSUE }\end{array}$ & 710-739 & 0 & 3 & 0 \\
\hline -Arthropathies & $710-719$ & 0 & 2 & 0 \\
\hline -Rheumatism, Excluding Back & \begin{tabular}{|l|}
$725-729$ \\
\end{tabular} & 0 & 1 & 0 \\
\hline INJURY \& POISONING & $800-999$ & 1 & 1 & 0 \\
\hline -Fracture - Lower Limb & 820-829 & 1 & 0 & 0 \\
\hline -Dislocation & $830-839$ & 0 & 0 & 0 \\
\hline -Sprains \& Strains - Other & $840-845,848$ & 0 & 1 & 0 \\
\hline
\end{tabular}

*Only those diagnostic categories and gender/job category combinations with at least one occurrence appear in this table. 
Idaho National Laboratory 2007

Absence Data

Appendix I. Number of Diagnoses in Each Diagnostic Category by Gender and Job Category*

\begin{tabular}{|c|c|c|c|c|c|}
\hline & & \multicolumn{4}{|c|}{ Women } \\
\hline & & \multicolumn{3}{|c|}{ Job Category } & \multirow[b]{2}{*}{ TOTAL } \\
\hline & & Service & Security and Fire & Crafts & \\
\hline Diagnostic Category & ICD-9-CM Code & \multirow[b]{2}{*}{0} & \multirow[b]{2}{*}{0} & \multirow[b]{2}{*}{1} & \multirow[b]{2}{*}{1} \\
\hline MALIGNANT NEOPLASMS & $140-208,230-234$ & & & & \\
\hline -Breast & 174-175 & 0 & 0 & 1 & 1 \\
\hline NERVOUS SYSTEM (NS) \& SENSE ORGANS & 320-389 & 1 & 0 & 0 & 1 \\
\hline -Disorders of Eye & $360-379$ & 1 & 0 & 0 & 1 \\
\hline CIRCULATORY SYSTEM & $390-459$ & 0 & 0 & 0 & 1 \\
\hline -Cerebrovascular Dis & $430-438$ & 0 & 0 & 0 & 1 \\
\hline RESPIRATORY SYSTEM & $460-519$ & 1 & 0 & 1 & 8 \\
\hline -Acute Respiratory Infections & $460-466$ & 0 & 0 & 1 & 4 \\
\hline -Other Dis Upper Respiratory Tract & $470-478$ & 1 & 0 & 0 & 2 \\
\hline -Pneumonia \& Influenza & $480-487$ & 0 & 0 & 0 & 2 \\
\hline DIGESTIVE SYSTEM & $520-579$ & 1 & 0 & 1 & 5 \\
\hline -Esophagus, Stomach, Duodenum & $530-537$ & 0 & 0 & 0 & 2 \\
\hline -Enteritis, Colitis & $555-558$ & 1 & 0 & 0 & 1 \\
\hline -Other Digestive Dis & $570-579$ & 0 & 0 & 1 & 2 \\
\hline GENITOURINARY SYSTEM & $580-629$ & 0 & 0 & 0 & 2 \\
\hline -Breast Disorders & $610-611$ & 0 & 0 & 0 & 1 \\
\hline -Other Female Disorders & $617-629$ & 0 & 0 & 0 & 1 \\
\hline $\begin{array}{l}\text { MUSCULOSKELETAL \& CONNECTIVE } \\
\text { TISSUE }\end{array}$ & 710-739 & 0 & 0 & 0 & 3 \\
\hline -Arthropathies & $710-719$ & 0 & 0 & 0 & 2 \\
\hline -Rheumatism, Excluding Back & $725-729$ & 0 & 0 & 0 & 1 \\
\hline INJURY \& POISONING & $800-999$ & 1 & 1 & 1 & 5 \\
\hline -Fracture - Lower Limb & $820-829$ & 0 & 1 & 0 & 2 \\
\hline -Dislocation & 830-839 & 1 & 0 & 0 & 1 \\
\hline -Sprains \& Strains - Other & $840-845,848$ & 0 & 0 & 1 & 2 \\
\hline
\end{tabular}

\begin{tabular}{|c|c|c|c|c|c|c|c|}
\hline & \multicolumn{7}{|c|}{ Women } \\
\hline & \multicolumn{6}{|c|}{ Job Category } & \multirow[b]{2}{*}{ TOTAL } \\
\hline & Professional & $\begin{array}{c}\text { Administrative } \\
\text { Support }\end{array}$ & Technical Support & Service & Security and Fire & Crafts & \\
\hline Diagnostic Category & \multirow[b]{2}{*}{3} & \multirow[b]{2}{*}{11} & \multirow[b]{2}{*}{3} & \multirow[b]{2}{*}{4} & \multirow[b]{2}{*}{1} & \multirow[b]{2}{*}{4} & \multirow[b]{2}{*}{26} \\
\hline TOTAL & & & & & & & \\
\hline
\end{tabular}

*Only those diagnostic categories and gender/job category combinations with at least one occurrence appear in this table. 
Idaho National Laboratory 2007

Absence Data

Appendix I. Number of Diagnoses in Each Diagnostic Category by Gender and Job Category*

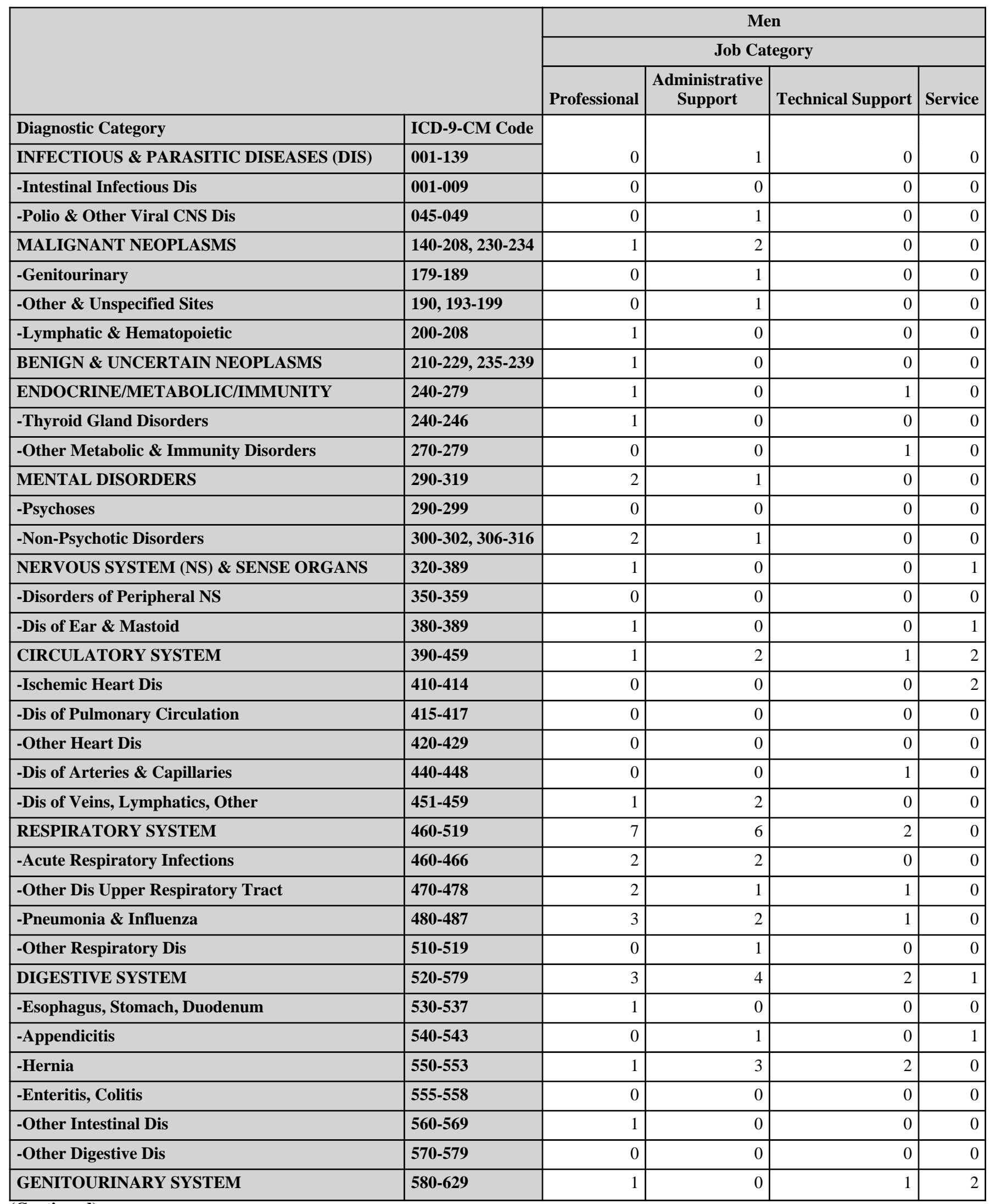

(Continued)

*Only those diagnostic categories and gender/job category combinations with at least one occurrence appear in this table. 
Idaho National Laboratory 2007

Absence Data

Appendix I. Number of Diagnoses in Each Diagnostic Category by Gender and Job Category*

\begin{tabular}{|c|c|c|c|c|c|}
\hline & & \multicolumn{4}{|c|}{ Men } \\
\hline & & \multicolumn{3}{|c|}{ Job Category } & \multirow[b]{2}{*}{ TOTAL } \\
\hline & & Security and Fire & Crafts & Line Operators & \\
\hline Diagnostic Category & ICD-9-CM Code & \multirow[b]{2}{*}{0} & \multirow[b]{2}{*}{1} & \multirow[b]{2}{*}{0} & \multirow[b]{2}{*}{2} \\
\hline INFECTIOUS \& PARASITIC DISEASES (DIS) & 001-139 & & & & \\
\hline -Intestinal Infectious Dis & \begin{tabular}{|c|}
$001-009$ \\
\end{tabular} & 0 & 1 & 0 & 1 \\
\hline -Polio \& Other Viral CNS Dis & 045-049 & 0 & 0 & 0 & 1 \\
\hline MALIGNANT NEOPLASMS & $140-208,230-234$ & 0 & 0 & 1 & 4 \\
\hline -Genitourinary & 179-189 & 0 & 0 & 1 & 2 \\
\hline -Other \& Unspecified Sites & 190, 193-199 & 0 & 0 & 0 & 1 \\
\hline -Lymphatic \& Hematopoietic & $200-208$ & 0 & 0 & 0 & 1 \\
\hline BENIGN \& UNCERTAIN NEOPLASMS & $210-229,235-239$ & 0 & 0 & 0 & 1 \\
\hline ENDOCRINE/METABOLIC/IMMUNITY & $240-279$ & 0 & 0 & 0 & 2 \\
\hline -Thyroid Gland Disorders & $240-246$ & 0 & 0 & 0 & 1 \\
\hline -Other Metabolic \& Immunity Disorders & $270-279$ & 0 & 0 & 0 & 1 \\
\hline MENTAL DISORDERS & 290-319 & 0 & 3 & 0 & 6 \\
\hline -Psychoses & $290-299$ & 0 & 2 & 0 & 2 \\
\hline -Non-Psychotic Disorders & $300-302,306-316$ & 0 & 1 & 0 & 4 \\
\hline NERVOUS SYSTEM (NS) \& SENSE ORGANS & 320-389 & 0 & 3 & 0 & 5 \\
\hline -Disorders of Peripheral NS & 350-359 & 0 & 2 & 0 & 2 \\
\hline -Dis of Ear \& Mastoid & 380-389 & 0 & 1 & 0 & 3 \\
\hline CIRCULATORY SYSTEM & $390-459$ & 4 & 3 & 1 & 14 \\
\hline -Ischemic Heart Dis & $410-414$ & 4 & 0 & 0 & 6 \\
\hline -Dis of Pulmonary Circulation & $415-417$ & 0 & 1 & 0 & 1 \\
\hline -Other Heart Dis & $420-429$ & 0 & 1 & 1 & 2 \\
\hline -Dis of Arteries \& Capillaries & $440-448$ & 0 & 0 & 0 & 1 \\
\hline -Dis of Veins, Lymphatics, Other & $451-459$ & 0 & 1 & 0 & 4 \\
\hline RESPIRATORY SYSTEM & $460-519$ & 3 & 2 & 1 & 21 \\
\hline -Acute Respiratory Infections & $460-466$ & 1 & 1 & 1 & 7 \\
\hline -Other Dis Upper Respiratory Tract & $470-478$ & 0 & 0 & 0 & 4 \\
\hline -Pneumonia \& Influenza & $480-487$ & 2 & 1 & 0 & 9 \\
\hline -Other Respiratory Dis & $510-519$ & 0 & 0 & 0 & 1 \\
\hline DIGESTIVE SYSTEM & $520-579$ & 0 & 3 & 4 & 17 \\
\hline -Esophagus, Stomach, Duodenum & $530-537$ & 0 & 0 & 0 & 1 \\
\hline -Appendicitis & $540-543$ & 0 & 0 & 0 & 2 \\
\hline -Hernia & $550-553$ & 0 & 2 & 3 & 11 \\
\hline -Enteritis, Colitis & $555-558$ & 0 & 0 & 1 & 1 \\
\hline -Other Intestinal Dis & $560-569$ & 0 & 0 & 0 & 1 \\
\hline -Other Digestive Dis & $570-579$ & 0 & 1 & 0 & 1 \\
\hline GENITOURINARY SYSTEM & $580-629$ & 0 & 0 & 0 & 4 \\
\hline
\end{tabular}

(Continued)

*Only those diagnostic categories and gender/job category combinations with at least one occurrence appear in this table. 
Idaho National Laboratory 2007

Absence Data

Appendix I. Number of Diagnoses in Each Diagnostic Category by Gender and Job Category*

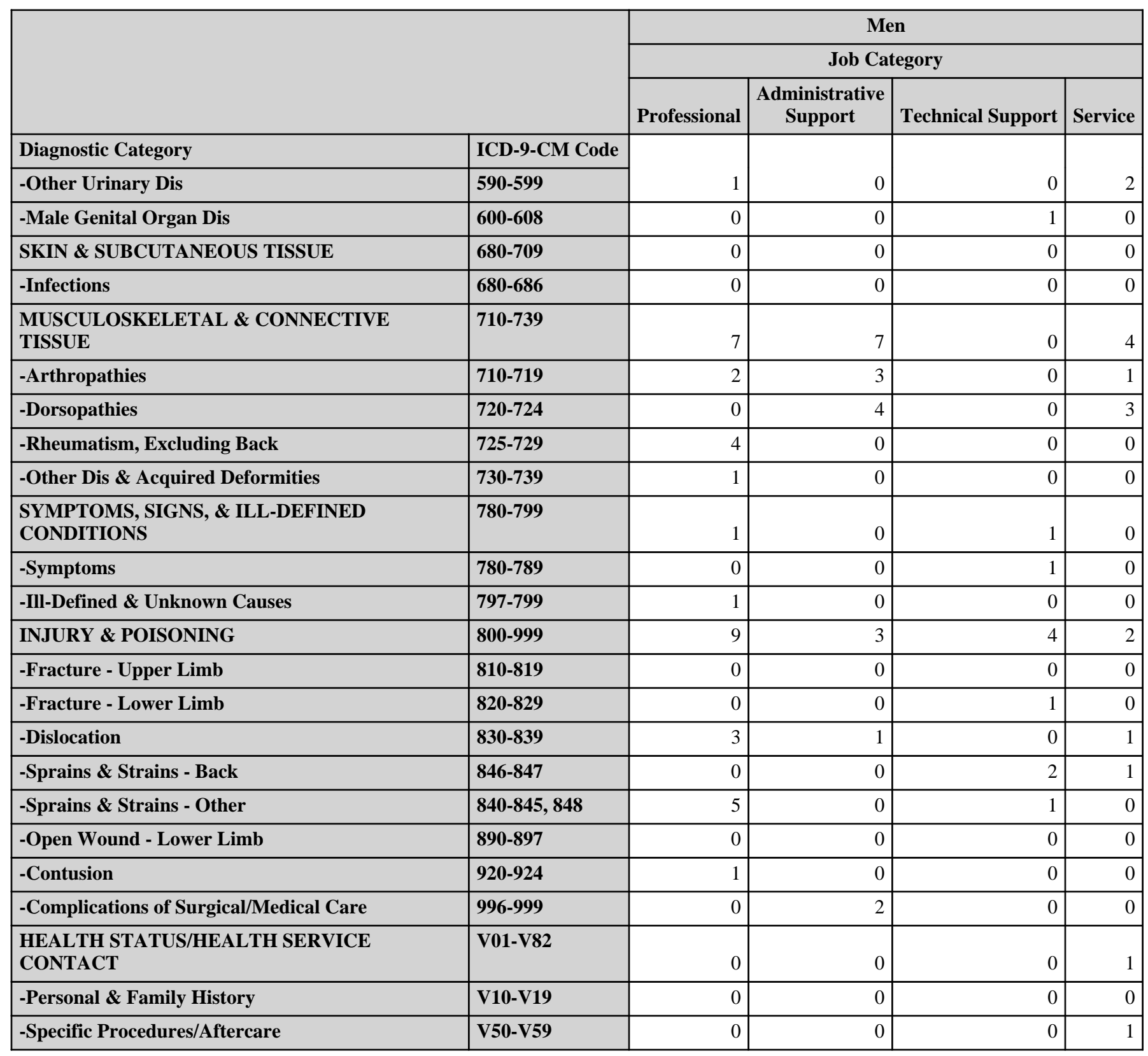

*Only those diagnostic categories and gender/job category combinations with at least one occurrence appear in this table. 
Idaho National Laboratory 2007

Absence Data

Appendix I. Number of Diagnoses in Each Diagnostic Category by Gender and Job Category*

\begin{tabular}{|c|c|c|c|c|c|}
\hline & & \multicolumn{4}{|c|}{ Men } \\
\hline & & \multicolumn{3}{|c|}{ Job Category } & \multirow[b]{2}{*}{ TOTAL } \\
\hline & & Security and Fire & Crafts & Line Operators & \\
\hline Diagnostic Category & ICD-9-CM Code & \multirow[b]{2}{*}{0} & \multirow[b]{2}{*}{0} & \multirow[b]{2}{*}{0} & \multirow[b]{2}{*}{3} \\
\hline -Other Urinary Dis & $590-599$ & & & & \\
\hline -Male Genital Organ Dis & $600-608$ & 0 & 0 & 0 & 1 \\
\hline SKIN \& SUBCUTANEOUS TISSUE & $680-709$ & 0 & 1 & 0 & 1 \\
\hline -Infections & $680-686$ & 0 & 1 & 0 & 1 \\
\hline $\begin{array}{l}\text { MUSCULOSKELETAL \& CONNECTIVE } \\
\text { TISSUE }\end{array}$ & $710-739$ & 1 & 9 & 2 & 30 \\
\hline -Arthropathies & $710-719$ & 0 & 3 & 2 & 11 \\
\hline -Dorsopathies & $720-724$ & 1 & 2 & 0 & 10 \\
\hline -Rheumatism, Excluding Back & 725-729 & 0 & 3 & 0 & 7 \\
\hline -Other Dis \& Acquired Deformities & 730-739 & 0 & 1 & 0 & 2 \\
\hline $\begin{array}{l}\text { SYMPTOMS, SIGNS, \& ILL-DEFINED } \\
\text { CONDITIONS }\end{array}$ & $780-799$ & 0 & 0 & 1 & 3 \\
\hline -Symptoms & $780-789$ & 0 & 0 & 1 & 2 \\
\hline -Ill-Defined \& Unknown Causes & 797-799 & 0 & 0 & 0 & 1 \\
\hline INJURY \& POISONING & $800-999$ & 1 & 8 & 1 & 28 \\
\hline -Fracture - Upper Limb & $810-819$ & 0 & 0 & 1 & 1 \\
\hline -Fracture - Lower Limb & $820-829$ & 0 & 1 & 0 & 2 \\
\hline -Dislocation & 830-839 & 0 & 3 & 0 & 8 \\
\hline -Sprains \& Strains - Back & 846-847 & 0 & 1 & 0 & 4 \\
\hline -Sprains \& Strains - Other & $840-845,848$ & 1 & 2 & 0 & 9 \\
\hline -Open Wound - Lower Limb & 890-897 & 0 & 1 & 0 & 1 \\
\hline -Contusion & $920-924$ & 0 & 0 & 0 & 1 \\
\hline -Complications of Surgical/Medical Care & 996-999 & 0 & 0 & 0 & 2 \\
\hline $\begin{array}{l}\text { HEALTH STATUS/HEALTH SERVICE } \\
\text { CONTACT }\end{array}$ & V01-V82 & 0 & 0 & 1 & 2 \\
\hline -Personal \& Family History & V10-V19 & 0 & 0 & 1 & 1 \\
\hline -Specific Procedures/Aftercare & V50-V59 & 0 & 0 & 0 & 1 \\
\hline
\end{tabular}

\begin{tabular}{|c|c|c|c|c|c|c|c|c|}
\hline & \multicolumn{8}{|c|}{ Men } \\
\hline & \multicolumn{7}{|c|}{ Job Category } & \multirow[b]{2}{*}{ TOTAL } \\
\hline & Professional & $\begin{array}{c}\text { Administrative } \\
\text { Support }\end{array}$ & $\begin{array}{l}\text { Technical } \\
\text { Support }\end{array}$ & Service & $\begin{array}{c}\text { Security and } \\
\text { Fire }\end{array}$ & Crafts & $\begin{array}{c}\text { Line } \\
\text { Operators }\end{array}$ & \\
\hline Diagnostic Category & \multirow[b]{2}{*}{35} & \multirow[b]{2}{*}{26} & \multirow[b]{2}{*}{12} & \multirow[b]{2}{*}{13} & \multirow[b]{2}{*}{9} & \multirow[b]{2}{*}{33} & \multirow[b]{2}{*}{12} & \multirow[b]{2}{*}{140} \\
\hline TOTAL & & & & & & & & \\
\hline
\end{tabular}

*Only those diagnostic categories and gender/job category combinations with at least one occurrence appear in this table. 
Idaho National Laboratory 2007

Absence Data

Appendix J. Total Number of Calendar Days Absent in Each Diagnostic Category by Gender and Job Category*

\begin{tabular}{|c|c|c|c|c|}
\hline & & \multicolumn{3}{|c|}{ Women } \\
\hline & & \multicolumn{3}{|c|}{ Job Category } \\
\hline & & Professional & $\begin{array}{l}\text { Administrative } \\
\text { Support }\end{array}$ & Technical Support \\
\hline Diagnostic Category & ICD-9-CM Code & \multirow[b]{2}{*}{0} & \multirow[b]{2}{*}{0} & \multirow[b]{2}{*}{0} \\
\hline MALIGNANT NEOPLASMS & $140-208,230-234$ & & & \\
\hline NERVOUS SYSTEM (NS) \& SENSE ORGANS & $320-389$ & 0 & 0 & $\overline{0}$ \\
\hline CIRCULATORY SYSTEM & $390-459$ & 0 & 43 & 0 \\
\hline RESPIRATORY SYSTEM & $460-519$ & 16 & 32 & 18 \\
\hline DIGESTIVE SYSTEM & $520-579$ & 0 & 13 & 13 \\
\hline GENITOURINARY SYSTEM & $580-629$ & 11 & 30 & 0 \\
\hline $\begin{array}{l}\text { MUSCULOSKELETAL \& CONNECTIVE } \\
\text { TISSUE }\end{array}$ & 710-739 & 0 & 63 & 0 \\
\hline INJURY \& POISONING & $800-999$ & 42 & 18 & 0 \\
\hline
\end{tabular}

\begin{tabular}{|c|c|c|c|c|c|}
\hline & & \multicolumn{4}{|c|}{ Women } \\
\hline & & \multicolumn{3}{|c|}{ Job Category } & \multirow[b]{2}{*}{ TOTAL } \\
\hline & & Service & Security and Fire & Crafts & \\
\hline Diagnostic Category & ICD-9-CM Code & \multirow[b]{2}{*}{0} & \multirow[b]{2}{*}{0} & \multirow[b]{2}{*}{91} & \multirow[b]{2}{*}{91} \\
\hline MALIGNANT NEOPLASMS & $140-208,230-234$ & & & & \\
\hline NERVOUS SYSTEM (NS) \& SENSE ORGANS & $320-389$ & 20 & 0 & 0 & 20 \\
\hline CIRCULATORY SYSTEM & 390-459 & 0 & 0 & 0 & 43 \\
\hline RESPIRATORY SYSTEM & $460-519$ & 7 & 0 & 19 & 92 \\
\hline DIGESTIVE SYSTEM & 520-579 & 7 & 0 & 17 & 50 \\
\hline GENITOURINARY SYSTEM & 580-629 & 0 & 0 & 0 & 41 \\
\hline $\begin{array}{l}\text { MUSCULOSKELETAL \& CONNECTIVE } \\
\text { TISSUE }\end{array}$ & 710-739 & 0 & 0 & 0 & 63 \\
\hline INJURY \& POISONING & $800-999$ & 18 & 73 & 19 & 170 \\
\hline
\end{tabular}

\footnotetext{
*Absences with >1 ICD-9-CM code in the same diagnostic category were counted only once. Only those diagnostic categories and gender/job category combinations with at least one occurrence appear in this table.
} 
Idaho National Laboratory 2007

Absence Data

Appendix J. Total Number of Calendar Days Absent in Each Diagnostic Category by Gender and Job Category*

\begin{tabular}{|c|c|c|c|c|c|}
\hline & & \multicolumn{4}{|c|}{ Men } \\
\hline & & \multicolumn{4}{|c|}{ Job Category } \\
\hline & & Professional & $\underset{\text { Support }}{\text { Administrative }}$ & Technical Support & Service \\
\hline Diagnostic Category & ICD-9-CM Code & \multirow[b]{2}{*}{0} & \multirow[b]{2}{*}{24} & \multirow[b]{2}{*}{0} & \multirow[b]{2}{*}{0} \\
\hline INFECTIOUS \& PARASITIC DISEASES (DIS) & 001-139 & & & & \\
\hline MALIGNANT NEOPLASMS & $140-208,230-234$ & 14 & 118 & 0 & 0 \\
\hline BENIGN \& UNCERTAIN NEOPLASMS & 210-229, 235-239 & 26 & 0 & 0 & 0 \\
\hline ENDOCRINE/METABOLIC/IMMUNITY & $240-279$ & 19 & 0 & 20 & 0 \\
\hline MENTAL DISORDERS & $290-319$ & 15 & 52 & 0 & 0 \\
\hline NERVOUS SYSTEM (NS) \& SENSE ORGANS & 320-389 & 25 & 0 & 0 & 27 \\
\hline CIRCULATORY SYSTEM & $390-459$ & 42 & 162 & 112 & 136 \\
\hline RESPIRATORY SYSTEM & $460-519$ & 127 & 65 & 48 & 0 \\
\hline DIGESTIVE SYSTEM & 520-579 & 82 & 82 & 47 & 47 \\
\hline GENITOURINARY SYSTEM & $580-629$ & 12 & 0 & 35 & 18 \\
\hline SKIN \& SUBCUTANEOUS TISSUE & 680-709 & 0 & 0 & 0 & 0 \\
\hline $\begin{array}{l}\text { MUSCULOSKELETAL \& CONNECTIVE } \\
\text { TISSUE }\end{array}$ & 710-739 & 229 & 366 & 0 & 214 \\
\hline $\begin{array}{l}\text { SYMPTOMS, SIGNS, \& ILL-DEFINED } \\
\text { CONDITIONS }\end{array}$ & 780-799 & 9 & 0 & 20 & 0 \\
\hline INJURY \& POISONING & $800-999$ & 211 & 135 & 124 & $\overline{43}$ \\
\hline
\end{tabular}
*Absences with >1 ICD-9-CM code in the same diagnostic category were counted only once. Only those diagnostic categories and gender/job
category combinations with at least one occurrence appear in this table. 
Idaho National Laboratory 2007

Absence Data

Appendix J. Total Number of Calendar Days Absent in Each Diagnostic Category by Gender and Job Category*

\begin{tabular}{|c|c|c|c|c|c|}
\hline & & \multicolumn{4}{|c|}{ Men } \\
\hline & & \multicolumn{3}{|c|}{ Job Category } & \multirow[b]{2}{*}{ TOTAI } \\
\hline & & Security and Fire & Crafts & Line Operators & \\
\hline Diagnostic Category & ICD-9-CM Code & \multirow[b]{2}{*}{0} & \multirow[b]{2}{*}{21} & \multirow[b]{2}{*}{0} & \multirow[b]{2}{*}{45} \\
\hline INFECTIOUS \& PARASITIC DISEASES (DIS) & 001-139 & & & & \\
\hline MALIGNANT NEOPLASMS & $140-208,230-234$ & 0 & 0 & 42 & 174 \\
\hline BENIGN \& UNCERTAIN NEOPLASMS & 210-229, 235-239 & 0 & 0 & 0 & 26 \\
\hline ENDOCRINE/METABOLIC/IMMUNITY & $240-279$ & 0 & 0 & 0 & 39 \\
\hline MENTAL DISORDERS & $290-319$ & 0 & 75 & 0 & 142 \\
\hline NERVOUS SYSTEM (NS) \& SENSE ORGANS & 320-389 & 0 & 61 & 0 & 113 \\
\hline CIRCULATORY SYSTEM & $390-459$ & 33 & 86 & 167 & 738 \\
\hline RESPIRATORY SYSTEM & $460-519$ & 49 & 129 & 22 & 440 \\
\hline DIGESTIVE SYSTEM & 520-579 & 0 & 53 & 225 & 536 \\
\hline GENITOURINARY SYSTEM & $580-629$ & 0 & 0 & 0 & 65 \\
\hline SKIN \& SUBCUTANEOUS TISSUE & 680-709 & 0 & 33 & 0 & 33 \\
\hline $\begin{array}{l}\text { MUSCULOSKELETAL \& CONNECTIVE } \\
\text { TISSUE }\end{array}$ & 710-739 & 17 & 444 & 146 & 1,416 \\
\hline $\begin{array}{l}\text { SYMPTOMS, SIGNS, \& ILL-DEFINED } \\
\text { CONDITIONS }\end{array}$ & 780-799 & 0 & 0 & 167 & 196 \\
\hline INJURY \& POISONING & $800-999$ & 41 & 264 & 16 & 834 \\
\hline
\end{tabular}

\footnotetext{
*Absences with >1 ICD-9-CM code in the same diagnostic category were counted only once. Only those diagnostic categories and gender/job category combinations with at least one occurrence appear in this table.
} 
Idaho National Laboratory 2007

Absence Data

Appendix K. Age-Adjusted IIIness and Injury Rates by Diagnostic Category*

Part 1. Men

\begin{tabular}{|c|c|c|c|c|c|}
\hline & & $\begin{array}{l}\text { Number of } \\
\text { Diagnoses }\end{array}$ & $\begin{array}{c}\text { Age-Adjusted } \\
\text { Rate per } 1,000 * *\end{array}$ & $\begin{array}{c}\text { Lower } 95 \% \\
\text { Confidence } \\
\text { Limit per 1,000 }\end{array}$ & $\begin{array}{c}\text { Upper } 95 \% \\
\text { Confidence } \\
\text { Limit per 1,000 }\end{array}$ \\
\hline Diagnostic Category & ICD-9-CM Code & & & & \\
\hline INFECTIOUS \& PARASITIC DISEASES (DIS) & 001-139 & 2 & 0.3 & 0.1 & 1.2 \\
\hline -Intestinal Infectious Dis & 001-009 & 1 & 0.2 & 0.0 & 1.2 \\
\hline -Polio \& Other Viral CNS Dis & \begin{tabular}{|c|c|}
$045-049$ \\
\end{tabular} & 1 & 0.1 & 0.0 & 0.8 \\
\hline MALIGNANT NEOPLASMS & $140-208,230-234$ & 4 & 0.5 & 0.2 & 1.4 \\
\hline -Genitourinary & 179-189 & 2 & 0.2 & 0.1 & 0.9 \\
\hline -Other \& Unspecified Sites & 190, 193-199 & 1 & 0.2 & 0.0 & 1.2 \\
\hline -Lymphatic \& Hematopoietic & $200-208$ & 1 & 0.1 & 0.0 & 0.8 \\
\hline BENIGN \& UNCERTAIN NEOPLASMS & $210-229,235-239$ & 1 & 0.3 & 0.0 & 2.0 \\
\hline ENDOCRINE/METABOLIC/IMMUNITY & 240-279 & 2 & 0.3 & 0.1 & 1.2 \\
\hline -Thyroid Gland Disorders & $240-246$ & 1 & 0.1 & 0.0 & 0.8 \\
\hline -Other Metabolic \& Immunity Disorders & $270-279$ & 1 & 0.2 & 0.0 & 1.2 \\
\hline MENTAL DISORDERS & 290-319 & 6 & 0.9 & 0.4 & 1.9 \\
\hline -Psychoses & 290-299 & 2 & 0.3 & 0.1 & 1.4 \\
\hline -Non-Psychotic Disorders & $300-302,306-316$ & 4 & 0.5 & 0.2 & 1.4 \\
\hline NERVOUS SYSTEM (NS) \& SENSE ORGANS & 320-389 & 5 & 0.6 & 0.2 & 1.3 \\
\hline -Disorders of Peripheral NS & $350-359$ & 2 & 0.2 & 0.1 & 0.9 \\
\hline -Dis of Ear \& Mastoid & $380-389$ & 3 & 0.3 & 0.1 & 1.0 \\
\hline CIRCULATORY SYSTEM & $390-459$ & 14 & 2.1 & 1.2 & 3.7 \\
\hline -Ischemic Heart Dis & $410-414$ & 6 & 1.0 & 0.4 & 2.4 \\
\hline -Dis of Pulmonary Circulation & $415-417$ & 1 & 0.1 & 0.0 & 0.8 \\
\hline -Other Heart Dis & $420-429$ & 2 & 0.2 & 0.1 & 0.9 \\
\hline -Dis of Arteries \& Capillaries & $440-448$ & 1 & 0.1 & 0.0 & 0.8 \\
\hline -Dis of Veins, Lymphatics, Other & $451-459$ & 4 & 0.6 & 0.2 & 1.8 \\
\hline RESPIRATORY SYSTEM & $460-519$ & 21 & 4.2 & 2.6 & 6.8 \\
\hline -Acute Respiratory Infections & $460-466$ & 7 & 1.6 & 0.7 & 3.6 \\
\hline -Other Dis Upper Respiratory Tract & $470-478$ & 4 & 0.7 & 0.2 & 1.9 \\
\hline -Pneumonia \& Influenza & $480-487$ & 9 & 1.9 & 0.9 & 3.9 \\
\hline -Other Respiratory Dis & $510-519$ & 1 & 0.1 & 0.0 & 0.8 \\
\hline DIGESTIVE SYSTEM & $520-579$ & 17 & 2.8 & 1.7 & 4.7 \\
\hline -Esophagus, Stomach, Duodenum & $530-537$ & 1 & 0.3 & 0.0 & 2.0 \\
\hline -Appendicitis & $540-543$ & 2 & 0.3 & 0.1 & 1.2 \\
\hline -Hernia & $550-553$ & 11 & 1.7 & 0.9 & 3.2 \\
\hline -Enteritis, Colitis & $555-558$ & 1 & 0.1 & 0.0 & 0.8 \\
\hline -Other Intestinal Dis & $560-569$ & 1 & 0.3 & 0.0 & 2.0 \\
\hline -Other Digestive Dis & $570-579$ & 1 & 0.2 & 0.0 & 1.2 \\
\hline
\end{tabular}

(Continued)

* Only those diagnostic categories with at least one occurrence appear in this table.

**Standardized to age distribution of 2000 U.S. population. 
Idaho National Laboratory 2007

Absence Data

Appendix K. Age-Adjusted Illness and Injury Rates by Diagnostic Category*

Part 1. Men

\begin{tabular}{|c|c|c|c|c|c|}
\hline & & $\begin{array}{l}\text { Number of } \\
\text { Diagnoses }\end{array}$ & $\begin{array}{c}\text { Age-Adjusted } \\
\text { Rate per } 1,000 * *\end{array}$ & $\begin{array}{c}\text { Lower } 95 \% \\
\text { Confidence } \\
\text { Limit per 1,000 }\end{array}$ & $\begin{array}{c}\text { Upper } 95 \% \\
\text { Confidence } \\
\text { Limit per 1,000 }\end{array}$ \\
\hline Diagnostic Category & ICD-9-CM Code & & & & \\
\hline GENITOURINARY SYSTEM & $580-629$ & 4 & 0.6 & 0.2 & 1.5 \\
\hline -Other Urinary Dis & $590-599$ & 3 & 0.4 & 0.1 & 1.3 \\
\hline -Male Genital Organ Dis & $600-608$ & 1 & 0.2 & 0.0 & 1.2 \\
\hline SKIN \& SUBCUTANEOUS TISSUE & $680-709$ & 1 & 0.1 & 0.0 & 0.8 \\
\hline -Infections & $680-686$ & 1 & 0.1 & 0.0 & 0.8 \\
\hline $\begin{array}{l}\text { MUSCULOSKELETAL \& CONNECTIVE } \\
\text { TISSUE }\end{array}$ & $710-739$ & 30 & 4.6 & 3.0 & 6.9 \\
\hline -Arthropathies & $710-719$ & 11 & 1.5 & 0.8 & 2.8 \\
\hline -Dorsopathies & $720-724$ & 10 & 1.9 & 0.9 & 4.0 \\
\hline -Rheumatism, Excluding Back & 725-729 & 7 & 0.9 & 0.4 & 1.9 \\
\hline -Other Dis \& Acquired Deformities & $730-739$ & 2 & 0.3 & 0.1 & 1.2 \\
\hline $\begin{array}{l}\text { SYMPTOMS, SIGNS, \& ILL-DEFINED } \\
\text { CONDITIONS }\end{array}$ & 780-799 & 3 & 0.4 & 0.1 & 1.3 \\
\hline -Symptoms & $780-789$ & 2 & 0.3 & 0.1 & 1.2 \\
\hline -Ill-Defined \& Unknown Causes & 797-799 & 1 & 0.1 & 0.0 & 0.8 \\
\hline INJURY \& POISONING & $800-999$ & 28 & 5.7 & 3.4 & 9.6 \\
\hline -Fracture - Upper Limb & 810-819 & 1 & 1.2 & 0.2 & 8.3 \\
\hline -Fracture - Lower Limb & $820-829$ & 2 & 0.3 & 0.1 & 1.2 \\
\hline -Dislocation & 830-839 & 8 & 1.6 & 0.7 & 3.5 \\
\hline -Sprains \& Strains - Back & 846-847 & 4 & 0.8 & 0.3 & 2.3 \\
\hline -Sprains \& Strains - Other & $840-845,848$ & 9 & 1.3 & 0.7 & 2.5 \\
\hline -Open Wound - Head, Neck, Trunk & 870-879 & 1 & 0.2 & 0.0 & 1.2 \\
\hline -Contusion & $920-924$ & 1 & 0.2 & 0.0 & 1.2 \\
\hline -Complications of Surgical/Medical Care & 996-999 & 2 & 0.2 & 0.1 & 0.9 \\
\hline Total & & 138 & 23.2 & 18.9 & 28.4 \\
\hline
\end{tabular}

* Only those diagnostic categories with at least one occurrence appear in this table.

**Standardized to age distribution of 2000 U.S. population. 
Idaho National Laboratory 2007

Absence Data

Appendix K. Age-Adjusted Illness and Injury Rates by Diagnostic Category*

Part 2. Women

\begin{tabular}{|c|c|c|c|c|c|}
\hline & & $\begin{array}{l}\text { Number of } \\
\text { Diagnoses }\end{array}$ & $\begin{array}{c}\text { Age-Adjusted } \\
\text { Rate per } 1,000 * *\end{array}$ & $\begin{array}{c}\text { Lower } 95 \% \\
\text { Confidence } \\
\text { Limit per 1,000 }\end{array}$ & $\begin{array}{c}\text { Upper 95\% } \\
\text { Confidence } \\
\text { Limit per 1,000 }\end{array}$ \\
\hline Diagnostic Category & ICD-9-CM Code & & & & \\
\hline MALIGNANT NEOPLASMS & $140-208,230-234$ & 1 & 0.4 & 0.1 & 2.6 \\
\hline -Breast & 174-175 & 1 & 0.4 & 0.1 & 2.6 \\
\hline NERVOUS SYSTEM (NS) \& SENSE ORGANS & 320-389 & 1 & 0.4 & 0.1 & 2.6 \\
\hline -Disorders of Eye & $360-379$ & 1 & 0.4 & 0.1 & 2.6 \\
\hline CIRCULATORY SYSTEM & $390-459$ & 1 & 1.0 & 0.1 & 7.3 \\
\hline -Cerebrovascular Dis & $430-438$ & 1 & 1.0 & 0.1 & 7.3 \\
\hline RESPIRATORY SYSTEM & $460-519$ & 8 & 9.6 & 3.9 & 23.7 \\
\hline -Acute Respiratory Infections & $460-466$ & 4 & 7.9 & 2.7 & 23.2 \\
\hline -Other Dis Upper Respiratory Tract & $470-478$ & 2 & 0.8 & 0.2 & 3.4 \\
\hline -Pneumonia \& Influenza & $480-487$ & 2 & 0.8 & 0.2 & 3.4 \\
\hline DIGESTIVE SYSTEM & $520-579$ & 5 & 3.5 & 1.3 & 8.9 \\
\hline -Esophagus, Stomach, Duodenum & $\mathbf{5 3 0 - 5 3 7}$ & 2 & 2.1 & 0.5 & 8.3 \\
\hline -Enteritis, Colitis & $555-558$ & 1 & 0.5 & 0.1 & 3.3 \\
\hline -Other Digestive Dis & $570-579$ & 2 & 0.9 & 0.2 & 3.7 \\
\hline GENITOURINARY SYSTEM & $580-629$ & 2 & 0.8 & 0.2 & 3.4 \\
\hline -Breast Disorders & $610-611$ & 1 & 0.4 & 0.1 & 2.6 \\
\hline -Other Female Disorders & $617-629$ & 1 & 0.5 & 0.1 & 3.3 \\
\hline $\begin{array}{l}\text { MUSCULOSKELETAL \& CONNECTIVE } \\
\text { TISSUE }\end{array}$ & 710-739 & 3 & 4.0 & 1.2 & 13.9 \\
\hline -Arthropathies & $710-719$ & 2 & 2.2 & 0.4 & 11.1 \\
\hline -Rheumatism, Excluding Back & 725-729 & 1 & 1.8 & 0.2 & 12.5 \\
\hline INJURY \& POISONING & $800-999$ & 5 & 2.0 & 0.8 & 4.7 \\
\hline -Fracture - Lower Limb & $820-829$ & 2 & 0.7 & 0.2 & 3.0 \\
\hline -Dislocation & 830-839 & 1 & 0.4 & 0.1 & 2.6 \\
\hline -Sprains \& Strains - Other & $840-845,848$ & 2 & 0.8 & 0.2 & 3.4 \\
\hline Total & & 26 & 21.7 & 13.1 & 35.9 \\
\hline
\end{tabular}

* Only those diagnostic categories with at least one occurrence appear in this table.

**Standardized to age distribution of 2000 U.S. population. 
Idaho National Laboratory 2007

Absence Data

Appendix K. Age-Adjusted Illness and Injury Rates by Diagnostic Category*

Part 3. Men and Women

\begin{tabular}{|c|c|c|c|c|c|}
\hline & & $\begin{array}{c}\text { Number of } \\
\text { Diagnoses }\end{array}$ & $\begin{array}{c}\text { Age-Adjusted } \\
\text { Rate per } 1,000^{* *}\end{array}$ & $\begin{array}{c}\text { Lower } 95 \% \\
\text { Confidence } \\
\text { Limit per 1,000 }\end{array}$ & $\begin{array}{c}\text { Upper } 95 \% \\
\text { Confidence } \\
\text { Limit per 1,000 }\end{array}$ \\
\hline Diagnostic Category & ICD-9-CM Code & & & & \\
\hline INFECTIOUS \& PARASITIC DISEASES (DIS) & 001-139 & 2 & 0.2 & 0.1 & 0.9 \\
\hline -Intestinal Infectious Dis & 001-009 & 1 & 0.1 & 0.0 & 0.9 \\
\hline -Polio \& Other Viral CNS Dis & 045-049 & 1 & 0.1 & 0.0 & 0.6 \\
\hline MALIGNANT NEOPLASMS & $140-208,230-234$ & 5 & 0.5 & 0.2 & 1.1 \\
\hline -Breast & 174-175 & 1 & 0.1 & 0.0 & 0.6 \\
\hline -Genitourinary & 179-189 & 2 & 0.2 & 0.0 & 0.7 \\
\hline -Other \& Unspecified Sites & $190,193-199$ & 1 & 0.1 & 0.0 & 0.9 \\
\hline -Lymphatic \& Hematopoietic & $200-208$ & 1 & 0.1 & 0.0 & 0.6 \\
\hline BENIGN \& UNCERTAIN NEOPLASMS & 210-229, 235-239 & 1 & 0.2 & 0.0 & 1.8 \\
\hline ENDOCRINE/METABOLIC/IMMUNITY & 240-279 & 2 & 0.2 & 0.1 & 0.9 \\
\hline -Thyroid Gland Disorders & $240-246$ & 1 & 0.1 & 0.0 & 0.6 \\
\hline -Other Metabolic \& Immunity Disorders & 270-279 & 1 & 0.1 & 0.0 & 0.9 \\
\hline MENTAL DISORDERS & 290-319 & 6 & 0.6 & 0.3 & 1.4 \\
\hline -Psychoses & 290-299 & 2 & 0.3 & 0.1 & 1.0 \\
\hline -Non-Psychotic Disorders & $300-302,306-316$ & 4 & 0.4 & 0.1 & 1.0 \\
\hline NERVOUS SYSTEM (NS) \& SENSE ORGANS & 320-389 & 6 & 0.5 & 0.2 & 1.2 \\
\hline -Disorders of Peripheral NS & 350-359 & 2 & 0.2 & 0.0 & 0.7 \\
\hline -Disorders of Eye & $360-379$ & 1 & 0.1 & 0.0 & 0.6 \\
\hline -Dis of Ear \& Mastoid & 380-389 & 3 & 0.3 & 0.1 & 0.8 \\
\hline CIRCULATORY SYSTEM & $390-459$ & 15 & 2.0 & 1.1 & 3.6 \\
\hline -Ischemic Heart Dis & $410-414$ & 6 & 0.8 & 0.3 & 2.1 \\
\hline -Dis of Pulmonary Circulation & 415-417 & 1 & 0.1 & 0.0 & 0.6 \\
\hline -Other Heart Dis & $420-429$ & 2 & 0.2 & 0.0 & 0.7 \\
\hline -Cerebrovascular Dis & $430-438$ & 1 & 0.3 & 0.0 & 2.2 \\
\hline -Dis of Arteries \& Capillaries & $440-448$ & 1 & 0.1 & 0.0 & 0.6 \\
\hline -Dis of Veins, Lymphatics, Other & 451-459 & 4 & 0.5 & 0.2 & 1.6 \\
\hline RESPIRATORY SYSTEM & $460-519$ & 29 & 6.2 & 3.8 & 10.4 \\
\hline -Acute Respiratory Infections & $460-466$ & 11 & 3.7 & 1.7 & 8.1 \\
\hline -Other Dis Upper Respiratory Tract & $470-478$ & 6 & 0.8 & 0.3 & 1.8 \\
\hline -Pneumonia \& Influenza & $480-487$ & 11 & 1.7 & 0.9 & 3.2 \\
\hline -Other Respiratory Dis & $510-519$ & 1 & 0.1 & 0.0 & 0.6 \\
\hline DIGESTIVE SYSTEM & $520-579$ & 22 & 3.3 & 2.1 & 5.3 \\
\hline -Esophagus, Stomach, Duodenum & $530-537$ & 3 & 0.9 & 0.3 & 2.7 \\
\hline -Appendicitis & $540-543$ & 2 & 0.2 & 0.1 & 0.9 \\
\hline -Hernia & $550-553$ & 11 & 1.4 & 0.7 & 2.6 \\
\hline
\end{tabular}

(Continued)

* Only those diagnostic categories with at least one occurrence appear in this table.

**Standardized to age distribution of 2000 U.S. population. 
Idaho National Laboratory 2007

Absence Data

Appendix K. Age-Adjusted Illness and Injury Rates by Diagnostic Category*

Part 3. Men and Women

\begin{tabular}{|c|c|c|c|c|c|}
\hline & & $\begin{array}{l}\text { Number of } \\
\text { Diagnoses }\end{array}$ & $\begin{array}{c}\text { Age-Adjusted } \\
\text { Rate per } 1,000 * *\end{array}$ & $\begin{array}{c}\text { Lower } 95 \% \\
\text { Confidence } \\
\text { Limit per 1,000 }\end{array}$ & $\begin{array}{c}\text { Upper } 95 \% \\
\text { Confidence } \\
\text { Limit per 1,000 }\end{array}$ \\
\hline Diagnostic Category & ICD-9-CM Code & & & & \\
\hline -Enteritis, Colitis & $555-558$ & 2 & 0.2 & 0.1 & 0.9 \\
\hline -Other Intestinal Dis & $560-569$ & 1 & 0.2 & 0.0 & 1.8 \\
\hline -Other Digestive Dis & $570-579$ & 3 & 0.4 & 0.1 & 1.2 \\
\hline GENITOURINARY SYSTEM & $580-629$ & 6 & 0.6 & 0.3 & 1.4 \\
\hline -Other Urinary Dis & $590-599$ & 3 & 0.3 & 0.1 & 0.9 \\
\hline -Male Genital Organ Dis & $600-608$ & 1 & 0.1 & 0.0 & 0.9 \\
\hline -Breast Disorders & $610-611$ & 1 & 0.1 & 0.0 & 0.6 \\
\hline -Other Female Disorders & $617-629$ & 1 & 0.1 & 0.0 & 0.9 \\
\hline SKIN \& SUBCUTANEOUS TISSUE & 680-709 & 1 & 0.1 & 0.0 & 0.6 \\
\hline -Infections & $680-686$ & 1 & 0.1 & 0.0 & 0.6 \\
\hline $\begin{array}{l}\text { MUSCULOSKELETAL \& CONNECTIVE } \\
\text { TISSUE }\end{array}$ & 710-739 & 33 & 4.1 & 2.8 & 6.0 \\
\hline -Arthropathies & 710-719 & 13 & 1.6 & 0.9 & 2.9 \\
\hline -Dorsopathies & 720-724 & 10 & 1.4 & 0.6 & 2.9 \\
\hline -Rheumatism, Excluding Back & 725-729 & 8 & 0.9 & 0.4 & 2.0 \\
\hline -Other Dis \& Acquired Deformities & 730-739 & 2 & 0.2 & 0.1 & 0.9 \\
\hline $\begin{array}{l}\text { SYMPTOMS, SIGNS, \& ILL-DEFINED } \\
\text { CONDITIONS }\end{array}$ & 780-799 & 3 & 0.3 & 0.1 & 0.9 \\
\hline -Symptoms & 780-789 & 2 & 0.2 & 0.1 & 0.9 \\
\hline -Ill-Defined \& Unknown Causes & 797-799 & 1 & 0.1 & 0.0 & 0.6 \\
\hline INJURY \& POISONING & 800-999 & 33 & 4.7 & 3.0 & 7.5 \\
\hline -Fracture - Upper Limb & 810-819 & 1 & 0.8 & 0.1 & 5.6 \\
\hline -Fracture - Lower Limb & 820-829 & 4 & 0.4 & 0.1 & 1.0 \\
\hline -Dislocation & 830-839 & 9 & 1.3 & 0.6 & 2.7 \\
\hline -Sprains \& Strains - Back & 846-847 & 4 & 0.7 & 0.2 & 2.0 \\
\hline -Sprains \& Strains - Other & $840-845,848$ & 11 & 1.2 & 0.7 & 2.2 \\
\hline -Open Wound - Head, Neck, Trunk & 870-879 & 1 & 0.1 & 0.0 & 0.9 \\
\hline -Contusion & 920-924 & 1 & 0.1 & 0.0 & 0.9 \\
\hline -Complications of Surgical/Medical Care & 996-999 & 2 & 0.2 & 0.0 & 0.7 \\
\hline Total & & 164 & 23.7 & 19.4 & 28.9 \\
\hline
\end{tabular}

* Only those diagnostic categories with at least one occurrence appear in this table.

**Standardized to age distribution of 2000 U.S. population. 
Idaho National Laboratory 2007

OSHA Data

Appendix L. Number of Workers with at Least One OSHA Event by Gender, Age, and Job Category*

\begin{tabular}{|c|c|c|c|c|c|c|c|c|c|c|}
\hline \multirow{3}{*}{ Job Category } & \multicolumn{4}{|c|}{ Women } & \multicolumn{5}{|c|}{ Men } & \multirow[b]{3}{*}{ TOTAL } \\
\hline & \multicolumn{3}{|c|}{ Age Group } & \multirow[b]{2}{*}{ TOTAL } & \multicolumn{4}{|c|}{ Age Group } & \multirow[b]{2}{*}{ TOTAL } & \\
\hline & $30-39$ & $40-49$ & $50+$ & & $16-29$ & $30-39$ & $40-49$ & $50+$ & & \\
\hline Professional & 0 & 1 & 0 & 1 & 1 & 1 & 1 & 4 & 7 & 8 \\
\hline Administrative Support & 1 & 0 & 4 & 5 & 0 & 0 & 0 & 3 & 3 & 8 \\
\hline Technical Support & 1 & 0 & 1 & 2 & 0 & 1 & 1 & 1 & 3 & 5 \\
\hline Service & 1 & 0 & 1 & 2 & 1 & 1 & 1 & 1 & 4 & 6 \\
\hline Security and Fire & 0 & 1 & 0 & 1 & 0 & 1 & 1 & 0 & 2 & 3 \\
\hline Crafts & 0 & 1 & 1 & 2 & 0 & 2 & 4 & 3 & 9 & 11 \\
\hline Line Operators & 0 & 0 & 0 & 0 & 0 & 0 & 0 & 1 & 1 & 1 \\
\hline TOTAL & 3 & 3 & 7 & 13 & 2 & 6 & 8 & 13 & 29 & 42 \\
\hline
\end{tabular}

*Only those job categories and gender/age combinations with at least one OSHA event appear in this table.

Appendix M. Total Number of Workdays Lost or with Restricted Activity from OSHA Events by Gender and Age

\begin{tabular}{|c|c|c|c|c|c|c|c|c|c|}
\hline \multirow[b]{2}{*}{ Age Group } & \multicolumn{3}{|c|}{ Women } & \multicolumn{3}{|c|}{ Men } & \multicolumn{3}{|c|}{ TOTAL } \\
\hline & $\begin{array}{l}\text { Number of } \\
\text { Events }\end{array}$ & $\begin{array}{c}\text { Days } \\
\text { Restricted }\end{array}$ & Days Lost & $\begin{array}{l}\text { Number of } \\
\text { Events }\end{array}$ & $\begin{array}{c}\text { Days } \\
\text { Restricted }\end{array}$ & Days Lost & $\begin{array}{l}\text { Number of } \\
\text { Events }\end{array}$ & $\begin{array}{c}\text { Days } \\
\text { Restricted }\end{array}$ & Days Lost \\
\hline $16-29$ & 0 & 0 & 0 & 2 & 0 & 0 & 2 & 0 & 0 \\
\hline $30-39$ & 3 & 78 & 76 & 6 & 305 & 158 & 9 & 383 & 234 \\
\hline $40-49$ & 3 & 0 & 0 & 8 & 82 & 188 & 11 & 82 & 188 \\
\hline $50+$ & 7 & 8 & 1 & 14 & 47 & 187 & 21 & 55 & 188 \\
\hline TOTAL & 13 & 86 & 77 & 30 & 434 & 533 & 43 & 520 & 610 \\
\hline
\end{tabular}

Appendix N. Total Number of Workdays Lost or with Restricted Activity from OSHA Events by Gender and Job Category*

\begin{tabular}{|c|c|c|c|c|c|c|c|c|c|}
\hline \multirow[b]{2}{*}{ Job Category } & \multicolumn{3}{|c|}{ Women } & \multicolumn{3}{|c|}{ Men } & \multicolumn{3}{|c|}{ TOTAL } \\
\hline & $\begin{array}{l}\text { Number of } \\
\text { Events }\end{array}$ & $\begin{array}{c}\text { Days } \\
\text { Restricted }\end{array}$ & Days Lost & $\begin{array}{l}\text { Number of } \\
\text { Events }\end{array}$ & $\begin{array}{c}\text { Days } \\
\text { Restricted }\end{array}$ & Days Lost & $\begin{array}{l}\text { Number of } \\
\text { Events }\end{array}$ & $\begin{array}{c}\text { Days } \\
\text { Restricted }\end{array}$ & Days Lost \\
\hline $\begin{array}{l}\text { Administrative } \\
\text { Support }\end{array}$ & 5 & 0 & 7 & 3 & 0 & 180 & 8 & 0 & 187 \\
\hline Technical Support & 2 & 0 & 0 & 3 & 106 & 119 & 5 & 106 & 119 \\
\hline Security and Fire & 1 & 0 & 0 & 2 & 104 & 0 & 3 & 104 & 0 \\
\hline Crafts & 2 & 6 & 1 & 9 & 203 & 181 & 11 & 209 & 182 \\
\hline Line Operators & 0 & 0 & 0 & 1 & 0 & 0 & 1 & 0 & 0 \\
\hline TOTAL & 13 & 86 & 77 & 30 & 434 & 533 & 43 & 520 & 610 \\
\hline
\end{tabular}

*Only those job categories with at least one OSHA event appear in this table. 
Idaho National Laboratory 2007

OSHA Data

Appendix O. Number of Diagnoses in Each Diagnostic Category by Gender and Age*

\begin{tabular}{|c|c|c|c|c|c|}
\hline & & \multicolumn{4}{|c|}{ Women } \\
\hline & & \multicolumn{3}{|c|}{ Age Group } & \multirow[b]{2}{*}{ TOTAL } \\
\hline & & $30-39$ & $40-49$ & $50+$ & \\
\hline Diagnostic Category & ICD-9-CM code & \multirow[b]{2}{*}{0} & \multirow[b]{2}{*}{0} & \multirow[b]{2}{*}{0} & \multirow[b]{2}{*}{0} \\
\hline NERVOUS SYSTEM (NS) \& SENSE ORGANS & 320-389 & & & & \\
\hline -Disorders of Eye & $360-379$ & 0 & 0 & 0 & 0 \\
\hline RESPIRATORY SYSTEM & $460-519$ & 0 & 1 & 0 & 1 \\
\hline -Other Respiratory Dis & $510-519$ & 0 & 1 & 0 & 1 \\
\hline $\begin{array}{l}\text { MUSCULOSKELETAL \& CONNECTIVE } \\
\text { TISSUE }\end{array}$ & 710-739 & 0 & 0 & 1 & 1 \\
\hline -Dorsopathies & 720-724 & 0 & 0 & 0 & 0 \\
\hline -Rheumatism, Excluding Back & 725-729 & 0 & 0 & 1 & 1 \\
\hline INJURY \& POISONING & $800-999$ & 3 & 4 & 6 & 13 \\
\hline -Fracture - Skull & 800-804 & 0 & 0 & 0 & 0 \\
\hline -Fracture - Upper Limb & 810-819 & 0 & 0 & 0 & 0 \\
\hline -Fracture - Lower Limb & $820-829$ & 0 & 0 & 0 & 0 \\
\hline -Sprains \& Strains - Back & 846-847 & 0 & 0 & 0 & 0 \\
\hline -Sprains \& Strains - Other & $840-845,848$ & 2 & 0 & 4 & 6 \\
\hline -Open Wound - Head, Neck, Trunk & $870-879$ & 0 & 1 & 0 & 1 \\
\hline -Open Wound - Upper Limb & 880-887 & 0 & 0 & 0 & 0 \\
\hline -Superficial Injury & 910-919 & 0 & 0 & 0 & 0 \\
\hline -Contusion & $920-924$ & 0 & 2 & 1 & 3 \\
\hline -Foreign Body Entering Orifice & 930-939 & 0 & 0 & 0 & 0 \\
\hline -Burns & $940-949$ & 0 & 1 & 0 & 1 \\
\hline -Complications \& Unspecified Injuries & 958-959 & 1 & 0 & 1 & 2 \\
\hline -Toxic Effects - Non-medicinal & 980-989 & 0 & 0 & 0 & 0 \\
\hline -Unspecified Effects - External Causes & $990-995$ & 0 & 0 & 0 & 0 \\
\hline
\end{tabular}

*Only those diagnostic categories and gender/age combinations with at least one OSHA event appear in this table. 
Idaho National Laboratory 2007

OSHA Data

Appendix O. Number of Diagnoses in Each Diagnostic Category by Gender and Age*

\begin{tabular}{|c|c|c|c|c|c|c|c|}
\hline & & \multicolumn{5}{|c|}{ Men } & \multirow[b]{3}{*}{ TOTAL } \\
\hline & & \multicolumn{4}{|c|}{ Age Group } & \multirow[b]{2}{*}{ TOTAL } & \\
\hline & & $16-29$ & 30 - 39 & 40 - 49 & $50+$ & & \\
\hline Diagnostic Category & ICD-9-CM code & & & & & & \\
\hline NERVOUS SYSTEM (NS) \& SENSE ORGANS & $320-389$ & 0 & 0 & 1 & 1 & 2 & 2 \\
\hline -Disorders of Eye & $360-379$ & 0 & 0 & 1 & 1 & 2 & 2 \\
\hline RESPIRATORY SYSTEM & $460-519$ & 1 & 0 & 0 & 0 & 1 & 2 \\
\hline -Other Respiratory Dis & $510-519$ & 1 & 0 & 0 & 0 & 1 & 2 \\
\hline $\begin{array}{l}\text { MUSCULOSKELETAL \& CONNECTIVE } \\
\text { TISSUE }\end{array}$ & \begin{tabular}{|c|}
$710-739$ \\
\end{tabular} & 0 & 0 & 1 & 0 & 1 & 2 \\
\hline -Dorsopathies & $720-724$ & 0 & 0 & 1 & 0 & 1 & 1 \\
\hline -Rheumatism, Excluding Back & 725-729 & 0 & 0 & 0 & 0 & 0 & 1 \\
\hline INJURY \& POISONING & $800-999$ & 2 & 7 & 6 & 13 & 28 & 41 \\
\hline -Fracture - Skull & $800-804$ & 0 & 0 & 0 & 1 & 1 & 1 \\
\hline -Fracture - Upper Limb & 810-819 & 0 & 1 & 0 & 1 & 2 & 2 \\
\hline -Fracture - Lower Limb & $820-829$ & 0 & 0 & 0 & 3 & 3 & 3 \\
\hline -Sprains \& Strains - Back & 846-847 & 0 & 3 & 0 & 1 & 4 & 4 \\
\hline -Sprains \& Strains - Other & $840-845,848$ & 0 & 2 & 3 & 0 & 5 & 11 \\
\hline -Open Wound - Head, Neck, Trunk & 870-879 & 0 & 0 & 0 & 0 & 0 & 1 \\
\hline -Open Wound - Upper Limb & \begin{tabular}{|l|}
$880-887$ \\
\end{tabular} & 1 & 0 & 1 & 5 & 7 & 7 \\
\hline -Superficial Injury & $910-919$ & 0 & 0 & 0 & 1 & 1 & 1 \\
\hline -Contusion & $920-924$ & 0 & 0 & 0 & 0 & 0 & 3 \\
\hline -Foreign Body Entering Orifice & \begin{tabular}{|c|}
$930-939$ \\
\end{tabular} & 0 & 0 & 1 & 0 & 1 & 1 \\
\hline -Burns & $940-949$ & 0 & 0 & 0 & 0 & 0 & 1 \\
\hline -Complications \& Unspecified Injuries & 958-959 & 0 & 0 & 0 & 1 & 1 & 3 \\
\hline -Toxic Effects - Non-medicinal & $980-989$ & 1 & 0 & 0 & 0 & 1 & 1 \\
\hline -Unspecified Effects - External Causes & $990-995$ & 0 & 1 & 1 & 0 & 2 & 2 \\
\hline
\end{tabular}

\begin{tabular}{|c|c|c|c|c|c|c|c|c|c|c|}
\hline & \multicolumn{4}{|c|}{ Women } & \multicolumn{5}{|c|}{ Men } & \multirow[b]{3}{*}{ TOTAL } \\
\hline & \multicolumn{3}{|c|}{ Age Group } & \multirow[b]{2}{*}{ TOTAL } & \multicolumn{4}{|c|}{ Age Group } & \multirow[b]{2}{*}{ TOTAL } & \\
\hline & $30-39$ & $40-49$ & $50+$ & & $16-29$ & 30 - 39 & $40-49$ & $50+$ & & \\
\hline Diagnostic Category & \multirow[b]{2}{*}{3} & \multirow[b]{2}{*}{5} & \multirow[b]{2}{*}{7} & \multirow[b]{2}{*}{15} & \multirow[b]{2}{*}{3} & \multirow[b]{2}{*}{7} & \multirow[b]{2}{*}{8} & \multirow[b]{2}{*}{14} & \multirow[b]{2}{*}{32} & \multirow[b]{2}{*}{47} \\
\hline TOTAL & & & & & & & & & & \\
\hline
\end{tabular}

*Only those diagnostic categories and gender/age combinations with at least one OSHA event appear in this table. 
Idaho National Laboratory 2007

OSHA Data

Appendix P. Number of Workdays Lost or with Restricted Activity in Each Diagnostic Category by Gender and Age*

\begin{tabular}{|c|c|c|c|c|c|c|c|}
\hline & & \multicolumn{6}{|c|}{ Women } \\
\hline & & \multicolumn{6}{|c|}{ Age Group } \\
\hline & & \multicolumn{2}{|c|}{30 - 39} & \multicolumn{2}{|c|}{$40-49$} & \multicolumn{2}{|c|}{$50+$} \\
\hline & & $\begin{array}{c}\text { Days } \\
\text { Restricted }\end{array}$ & Days Lost & $\begin{array}{c}\text { Days } \\
\text { Restricted }\end{array}$ & Days Lost & $\begin{array}{c}\text { Days } \\
\text { Restricted }\end{array}$ & Days Lost \\
\hline Diagnostic Category & ICD-9-CM Codes & \multirow[b]{2}{*}{0} & \multirow[b]{2}{*}{0} & \multirow[b]{2}{*}{0} & \multirow[b]{2}{*}{0} & \multirow[b]{2}{*}{0} & \multirow[b]{2}{*}{0} \\
\hline -Other Respiratory Dis & $510-519$ & & & & & & \\
\hline -Rheumatism, Excluding Back & 725-729 & 0 & 0 & 0 & 0 & 6 & 1 \\
\hline -Sprains \& Strains - Other & $840-845,848$ & 78 & 69 & 0 & 0 & 2 & 0 \\
\hline -Open Wound - Head, Neck, Trunk & 870-879 & 0 & 0 & 0 & 0 & 0 & 0 \\
\hline -Contusion & $920-924$ & 0 & 0 & 0 & 0 & 0 & 0 \\
\hline -Burns & $940-949$ & 0 & 0 & 0 & 0 & 0 & 0 \\
\hline $\begin{array}{l}\text {-Complications \& Unspecified } \\
\text { Injuries }\end{array}$ & 958-959 & 0 & 7 & 0 & 0 & 0 & 0 \\
\hline
\end{tabular}

\begin{tabular}{|c|c|c|c|c|c|c|c|c|c|}
\hline & & \multicolumn{8}{|c|}{ Men } \\
\hline & & \multicolumn{8}{|c|}{ Age Group } \\
\hline & & \multicolumn{2}{|c|}{$16-29$} & \multicolumn{2}{|c|}{30 - 39} & \multicolumn{2}{|c|}{$40-49$} & \multicolumn{2}{|c|}{$50+$} \\
\hline & & $\begin{array}{c}\text { Days } \\
\text { Restricted }\end{array}$ & $\begin{array}{l}\text { Days } \\
\text { Lost }\end{array}$ & $\begin{array}{c}\text { Days } \\
\text { Restricted }\end{array}$ & $\begin{array}{l}\text { Days } \\
\text { Lost }\end{array}$ & $\begin{array}{c}\text { Days } \\
\text { Restricted }\end{array}$ & $\begin{array}{l}\text { Days } \\
\text { Lost }\end{array}$ & $\begin{array}{c}\text { Days } \\
\text { Restricted }\end{array}$ & $\begin{array}{l}\text { Days } \\
\text { Lost }\end{array}$ \\
\hline Diagnostic Category & ICD-9-CM Codes & & & & & & & & \\
\hline -Disorders of Eye & $360-379$ & 0 & 0 & 0 & 0 & 0 & 0 & 0 & 0 \\
\hline -Other Respiratory Dis & $510-519$ & 0 & 0 & 0 & 0 & 0 & 0 & 0 & 0 \\
\hline -Dorsopathies & $720-724$ & 0 & 0 & 0 & 0 & 4 & 17 & 0 & 0 \\
\hline -Fracture - Skull & $800-804$ & 0 & 0 & 0 & 0 & 0 & 0 & 0 & 0 \\
\hline -Fracture - Upper Limb & 810-819 & 0 & 0 & 0 & 107 & 0 & 0 & 0 & 0 \\
\hline -Fracture - Lower Limb & 820-829 & 0 & 0 & 0 & 0 & 0 & 0 & 0 & 180 \\
\hline -Sprains \& Strains - Back & $846-847$ & 0 & 0 & 184 & 26 & 0 & 0 & 0 & 0 \\
\hline -Sprains \& Strains - Other & $840-845,848$ & 0 & 0 & 17 & 25 & 78 & 171 & 0 & 0 \\
\hline -Open Wound - Upper Limb & 880-887 & 0 & 0 & 0 & 0 & 0 & 0 & 47 & 7 \\
\hline -Superficial Injury & 910-919 & 0 & 0 & 0 & 0 & 0 & 0 & 0 & 0 \\
\hline -Foreign Body Entering Orifice & 930-939 & 0 & 0 & 0 & 0 & 0 & 0 & 0 & 0 \\
\hline $\begin{array}{l}\text {-Complications \& Unspecified } \\
\text { Injuries }\end{array}$ & 958-959 & 0 & 0 & 0 & 0 & 0 & 0 & 0 & 0 \\
\hline -Toxic Effects - Non-medicinal & 980-989 & 0 & 0 & 0 & 0 & 0 & 0 & 0 & 0 \\
\hline $\begin{array}{l}\text {-Unspecified Effects - External } \\
\text { Causes }\end{array}$ & $990-995$ & 0 & 0 & 104 & 0 & 0 & 0 & 0 & 0 \\
\hline
\end{tabular}

*OSHA events with >1 ICD-9-CM code in the same diagnostic category were counted only once. Only those diagnostic categories and gender/age combinations with at least one occurrence appear in this table. 
Idaho National Laboratory 2007

OSHA Data

Appendix Q. Number of Occurrences in Each Accident Category by Gender and Age*

\begin{tabular}{|c|c|c|c|c|c|c|c|c|c|c|}
\hline & & \multicolumn{3}{|c|}{ Women } & \multicolumn{5}{|c|}{ Men } & \multirow[b]{3}{*}{ TOTAI } \\
\hline & & \multicolumn{2}{|c|}{ Age Group } & \multirow[b]{2}{*}{ TOTAL } & \multicolumn{4}{|c|}{ Age Group } & \multirow[b]{2}{*}{ TOTAL } & \\
\hline & & 30 - 39 & $50+$ & & $16-29$ & $30-39$ & $40-49$ & $50+$ & & \\
\hline Accidental Poisoning - Non-medicinal & E860-E869 & 0 & 0 & 0 & 1 & 0 & 0 & 0 & 1 & 1 \\
\hline Falls & E880-E888 & 0 & 1 & 1 & 0 & 0 & 0 & 1 & 1 & 2 \\
\hline Other Accidents & E916-E928 & 1 & 1 & 2 & 0 & 2 & 1 & 5 & 8 & 10 \\
\hline
\end{tabular}

*Only those accident types and gender/age combinations with at least one occurrence appear in this table. 
Idaho National Laboratory 2007

OSHA Data

Appendix R. Number of Workdays Lost or with Restricted Activity in Each Accident Category by Gender and Age*

\begin{tabular}{|c|c|c|c|c|c|}
\hline & & \multicolumn{4}{|c|}{ Women } \\
\hline & & \multicolumn{4}{|c|}{ Age Group } \\
\hline & & \multicolumn{2}{|c|}{$30-39$} & \multicolumn{2}{|c|}{$50+$} \\
\hline & & $\begin{array}{c}\text { Days } \\
\text { Restricted }\end{array}$ & Days Lost & $\begin{array}{c}\text { Days } \\
\text { Restricted }\end{array}$ & Days Lost \\
\hline Type of Accident & E Codes & \multirow[b]{2}{*}{0} & \multirow[b]{2}{*}{0} & \multirow[b]{2}{*}{0} & \multirow[b]{2}{*}{0} \\
\hline Falls & E880-E888 & & & & \\
\hline Other Accidents & E916-E928 & 0 & 7 & 0 & 0 \\
\hline
\end{tabular}

\begin{tabular}{|c|c|c|c|c|c|c|c|c|c|}
\hline & & \multicolumn{8}{|c|}{ Men } \\
\hline & & \multicolumn{8}{|c|}{ Age Group } \\
\hline & & \multicolumn{2}{|c|}{$16-29$} & \multicolumn{2}{|c|}{$30-39$} & \multicolumn{2}{|c|}{$40-49$} & \multicolumn{2}{|c|}{$50+$} \\
\hline & & $\begin{array}{c}\text { Days } \\
\text { Restricted }\end{array}$ & $\begin{array}{l}\text { Days } \\
\text { Lost }\end{array}$ & $\begin{array}{c}\text { Days } \\
\text { Restricted }\end{array}$ & $\begin{array}{l}\text { Days } \\
\text { Lost }\end{array}$ & \begin{tabular}{|c|} 
Days \\
Restricted
\end{tabular} & $\begin{array}{l}\text { Days } \\
\text { Lost }\end{array}$ & $\begin{array}{c}\text { Days } \\
\text { Restricted }\end{array}$ & $\begin{array}{l}\text { Days } \\
\text { Lost }\end{array}$ \\
\hline Type of Accident & E Codes & \multirow[b]{2}{*}{0} & \multirow[b]{2}{*}{0} & \multirow[b]{2}{*}{0} & \multirow[b]{2}{*}{0} & \multirow[b]{2}{*}{0} & \multirow[b]{2}{*}{0} & \multirow[b]{2}{*}{0} & \multirow[b]{2}{*}{0} \\
\hline Accidental Poisoning - Non-medicinal & E860-E869 & & & & & & & & \\
\hline Falls & E880-E888 & 0 & 0 & 0 & 0 & 0 & 0 & 0 & 0 \\
\hline $\begin{array}{l}\text { Submersion/Suffocation/Foreign } \\
\text { Bodies }\end{array}$ & E910-E915 & 0 & 0 & 0 & 0 & 0 & 0 & 0 & 0 \\
\hline Other Accidents & E916-E928 & 0 & 0 & 104 & 107 & 0 & 0 & 0 & 0 \\
\hline
\end{tabular}

*OSHA events with $>1 \mathrm{E}$ code in the same accident type were counted only once. Only those accident types and gender/age combinations with at least one occurrence appear in this table. 
Idaho National Laboratory 2007

OSHA Data

Appendix S. Number of Diagnoses in Each Diagnostic Category by Gender and Job Category*

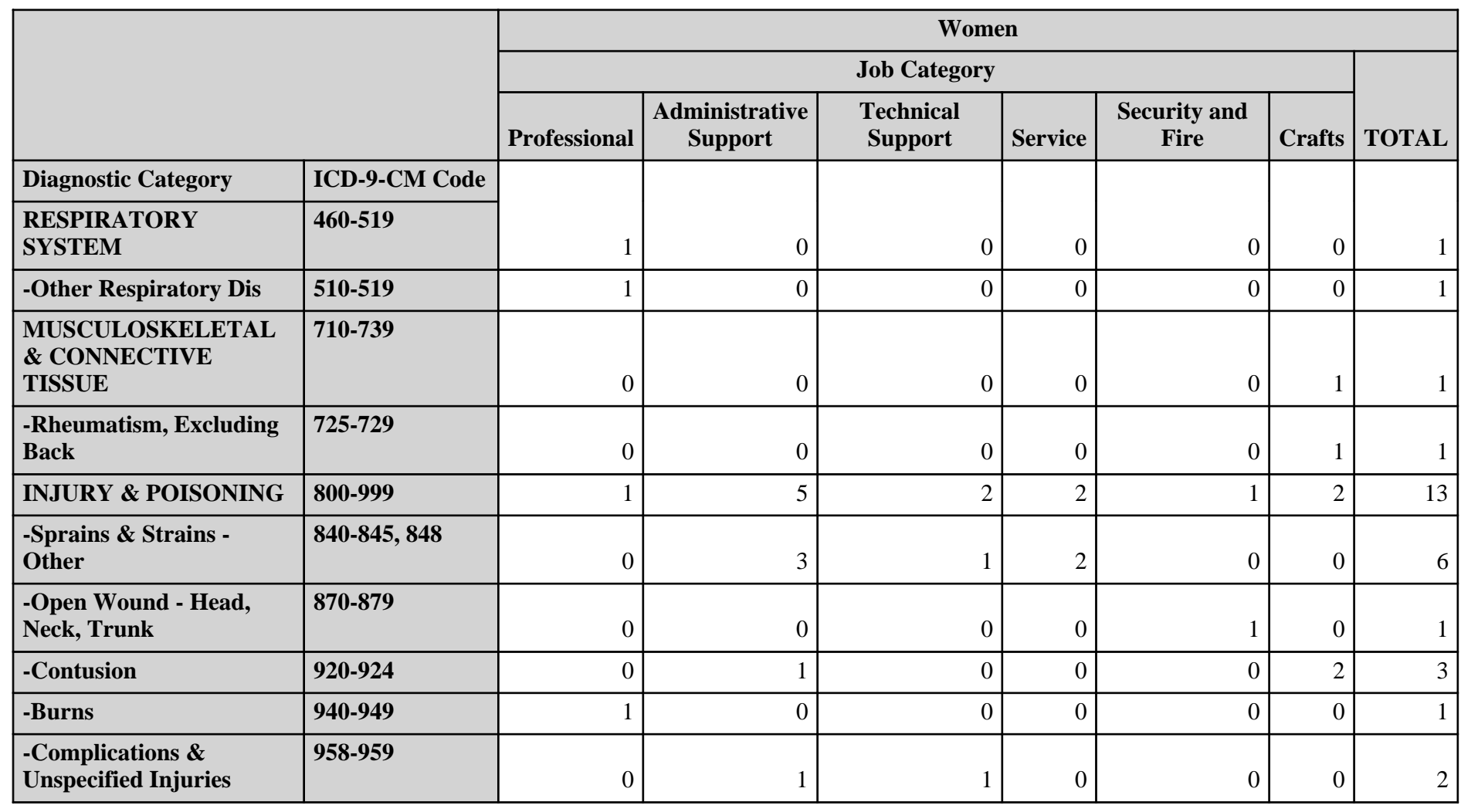

\begin{tabular}{|c|c|c|c|c|c|c|c|}
\hline & \multicolumn{7}{|c|}{ Women } \\
\hline & \multicolumn{6}{|c|}{ Job Category } & \multirow[b]{2}{*}{ TOTAL } \\
\hline & Professional & $\begin{array}{c}\text { Administrative } \\
\text { Support }\end{array}$ & Technical Support & Service & Security and Fire & Crafts & \\
\hline Diagnostic Category & \multirow[b]{2}{*}{2} & \multirow[b]{2}{*}{5} & \multirow[b]{2}{*}{2} & \multirow[b]{2}{*}{2} & \multirow[b]{2}{*}{1} & \multirow[b]{2}{*}{3} & \multirow[b]{2}{*}{15} \\
\hline TOTAL & & & & & & & \\
\hline
\end{tabular}

\begin{tabular}{|c|c|c|c|c|c|c|c|c|c|}
\hline & & \multicolumn{8}{|c|}{ Men } \\
\hline & & \multicolumn{7}{|c|}{ Job Category } & \multirow[b]{2}{*}{ TOTAL } \\
\hline & & Professional & $\begin{array}{c}\text { Administrative } \\
\text { Support }\end{array}$ & $\begin{array}{l}\text { Technical } \\
\text { Support }\end{array}$ & Service & $\begin{array}{l}\text { Security } \\
\text { and Fire }\end{array}$ & Crafts & $\begin{array}{c}\text { Line } \\
\text { Operators }\end{array}$ & \\
\hline Diagnostic Category & ICD-9-CM Code & & & & & & & & \\
\hline $\begin{array}{l}\text { NERVOUS SYSTEM } \\
\text { (NS) \& SENSE ORGANS }\end{array}$ & 320-389 & 0 & 0 & 0 & 1 & 0 & 1 & 0 & 2 \\
\hline -Disorders of Eye & $360-379$ & 0 & 0 & 0 & 1 & 0 & 1 & 0 & 2 \\
\hline $\begin{array}{l}\text { RESPIRATORY } \\
\text { SYSTEM }\end{array}$ & $460-519$ & 1 & 0 & 0 & 0 & 0 & 0 & 0 & 1 \\
\hline -Other Respiratory Dis & $510-519$ & 1 & 0 & 0 & 0 & 0 & 0 & 0 & 1 \\
\hline $\begin{array}{l}\text { MUSCULOSKELETAL } \\
\text { \& CONNECTIVE } \\
\text { TISSUE }\end{array}$ & $710-739$ & 0 & 0 & 0 & 1 & 0 & 0 & 0 & 1 \\
\hline -Dorsopathies & $720-724$ & 0 & 0 & 0 & 1 & 0 & 0 & 0 & 1 \\
\hline INJURY \& POISONING & $800-999$ & 8 & 3 & 3 & 3 & 2 & 8 & 1 & 28 \\
\hline
\end{tabular}

(Continued)

*Only those diagnostic categories and gender/job category combinations with at least one occurrence appear in this table. 
Idaho National Laboratory 2007

OSHA Data

Appendix S. Number of Diagnoses in Each Diagnostic Category by Gender and Job Category*

\begin{tabular}{|c|c|c|c|c|c|c|c|c|c|}
\hline & & \multicolumn{8}{|c|}{ Men } \\
\hline & & \multicolumn{7}{|c|}{ Job Category } & \multirow[b]{2}{*}{ TOTAL } \\
\hline & & Professional & $\begin{array}{c}\text { Administrative } \\
\text { Support }\end{array}$ & \begin{tabular}{|c|} 
Technical \\
Support
\end{tabular} & Service & $\begin{array}{l}\text { Security } \\
\text { and Fire }\end{array}$ & Crafts & $\begin{array}{c}\text { Line } \\
\text { Operators }\end{array}$ & \\
\hline Diagnostic Category & ICD-9-CM Code & \multirow[b]{2}{*}{0} & \multirow[b]{2}{*}{0} & \multirow[b]{2}{*}{0} & \multirow[b]{2}{*}{0} & \multirow[b]{2}{*}{0} & \multirow[b]{2}{*}{1} & \multirow[b]{2}{*}{0} & \multirow[b]{2}{*}{1} \\
\hline -Fracture - Skull & $800-804$ & & & & & & & & \\
\hline -Fracture - Upper Limb & \begin{tabular}{|c|}
$810-819$ \\
\end{tabular} & 1 & 0 & 0 & 0 & 0 & 1 & 0 & 2 \\
\hline -Fracture - Lower Limb & $820-829$ & 1 & 2 & 0 & 0 & 0 & 0 & 0 & 3 \\
\hline -Sprains \& Strains - Back & 846-847 & 1 & 0 & 1 & 0 & 0 & 2 & 0 & 4 \\
\hline $\begin{array}{l}\text {-Sprains \& Strains - } \\
\text { Other }\end{array}$ & $840-845,848$ & 2 & 0 & 1 & 1 & 0 & 1 & 0 & 5 \\
\hline $\begin{array}{l}\text {-Open Wound - Upper } \\
\text { Limb }\end{array}$ & $880-887$ & 1 & 1 & 1 & 1 & 0 & 2 & 1 & 7 \\
\hline -Superficial Injury & $910-919$ & 1 & 0 & 0 & 0 & 0 & 0 & 0 & 1 \\
\hline $\begin{array}{l}\text {-Foreign Body Entering } \\
\text { Orifice }\end{array}$ & \begin{tabular}{|c|}
$930-939$ \\
\end{tabular} & 0 & 0 & 0 & 0 & 0 & 1 & 0 & 1 \\
\hline $\begin{array}{l}\text {-Complications \& } \\
\text { Unspecified Injuries }\end{array}$ & \begin{tabular}{|c|}
$958-959$ \\
\end{tabular} & 0 & 0 & 0 & 1 & 0 & 0 & 0 & 1 \\
\hline $\begin{array}{l}\text {-Toxic } \\
\text { Effects - Non-medicinal }\end{array}$ & \begin{tabular}{|c|}
$980-989$ \\
\end{tabular} & 1 & 0 & 0 & 0 & 0 & 0 & 0 & 1 \\
\hline $\begin{array}{l}\text {-Unspecified } \\
\text { Effects - External Causes }\end{array}$ & \begin{tabular}{|c|}
$990-995$ \\
\end{tabular} & 0 & 0 & 0 & 0 & 2 & 0 & 0 & 2 \\
\hline
\end{tabular}

\begin{tabular}{|c|c|c|c|c|c|c|c|c|}
\hline & \multicolumn{8}{|c|}{ Men } \\
\hline & \multicolumn{7}{|c|}{ Job Category } & \multirow[b]{2}{*}{ TOTAL } \\
\hline & Professional & $\underset{\text { Support }}{\text { Administrative }}$ & $\begin{array}{l}\text { Technical } \\
\text { Support }\end{array}$ & Service & $\begin{array}{l}\text { Security and } \\
\text { Fire }\end{array}$ & Crafts & $\begin{array}{c}\text { Line } \\
\text { Operators }\end{array}$ & \\
\hline Diagnostic Category & \multirow[b]{2}{*}{9} & \multirow[b]{2}{*}{3} & \multirow[b]{2}{*}{3} & \multirow[b]{2}{*}{5} & \multirow[b]{2}{*}{2} & \multirow[b]{2}{*}{9} & \multirow[b]{2}{*}{1} & \multirow[b]{2}{*}{32} \\
\hline TOTAL & & & & & & & & \\
\hline
\end{tabular}

*Only those diagnostic categories and gender/job category combinations with at least one occurrence appear in this table. 
Idaho National Laboratory 2007

OSHA Data

Appendix T. Number of Workdays Lost or with Restricted Activity in Each Diagnostic Category by Gender and Job Category*

\begin{tabular}{|c|c|c|c|c|c|c|c|}
\hline & & \multicolumn{6}{|c|}{ Women } \\
\hline & & \multicolumn{6}{|c|}{ Job Category } \\
\hline & & \multicolumn{2}{|c|}{ Professional } & \multicolumn{2}{|c|}{$\begin{array}{l}\text { Administrative } \\
\text { Support }\end{array}$} & \multicolumn{2}{|c|}{ Technical Support } \\
\hline & & $\begin{array}{c}\text { Days } \\
\text { Restricted }\end{array}$ & Days Lost & $\begin{array}{c}\text { Days } \\
\text { Restricted }\end{array}$ & Days Lost & $\begin{array}{c}\text { Days } \\
\text { Restricted }\end{array}$ & Days Lost \\
\hline Diagnostic Category & ICD-9-CM Codes & \multirow[b]{2}{*}{0} & \multirow[b]{2}{*}{0} & \multirow[b]{2}{*}{0} & \multirow[b]{2}{*}{0} & \multirow[b]{2}{*}{0} & \multirow[b]{2}{*}{0} \\
\hline -Other Respiratory Dis & $510-519$ & & & & & & \\
\hline -Rheumatism, Excluding Back & $725-729$ & 0 & 0 & 0 & 0 & 0 & 0 \\
\hline -Sprains \& Strains - Other & $840-845,848$ & 0 & 0 & 0 & 0 & 0 & 0 \\
\hline -Open Wound - Head, Neck, Trunk & $870-879$ & 0 & 0 & 0 & 0 & 0 & 0 \\
\hline -Contusion & $920-924$ & 0 & 0 & 0 & 0 & 0 & 0 \\
\hline -Burns & $940-949$ & 0 & 0 & 0 & 0 & 0 & 0 \\
\hline $\begin{array}{l}\text {-Complications \& Unspecified } \\
\text { Injuries }\end{array}$ & 958-959 & 0 & 0 & 0 & 7 & 0 & 0 \\
\hline
\end{tabular}

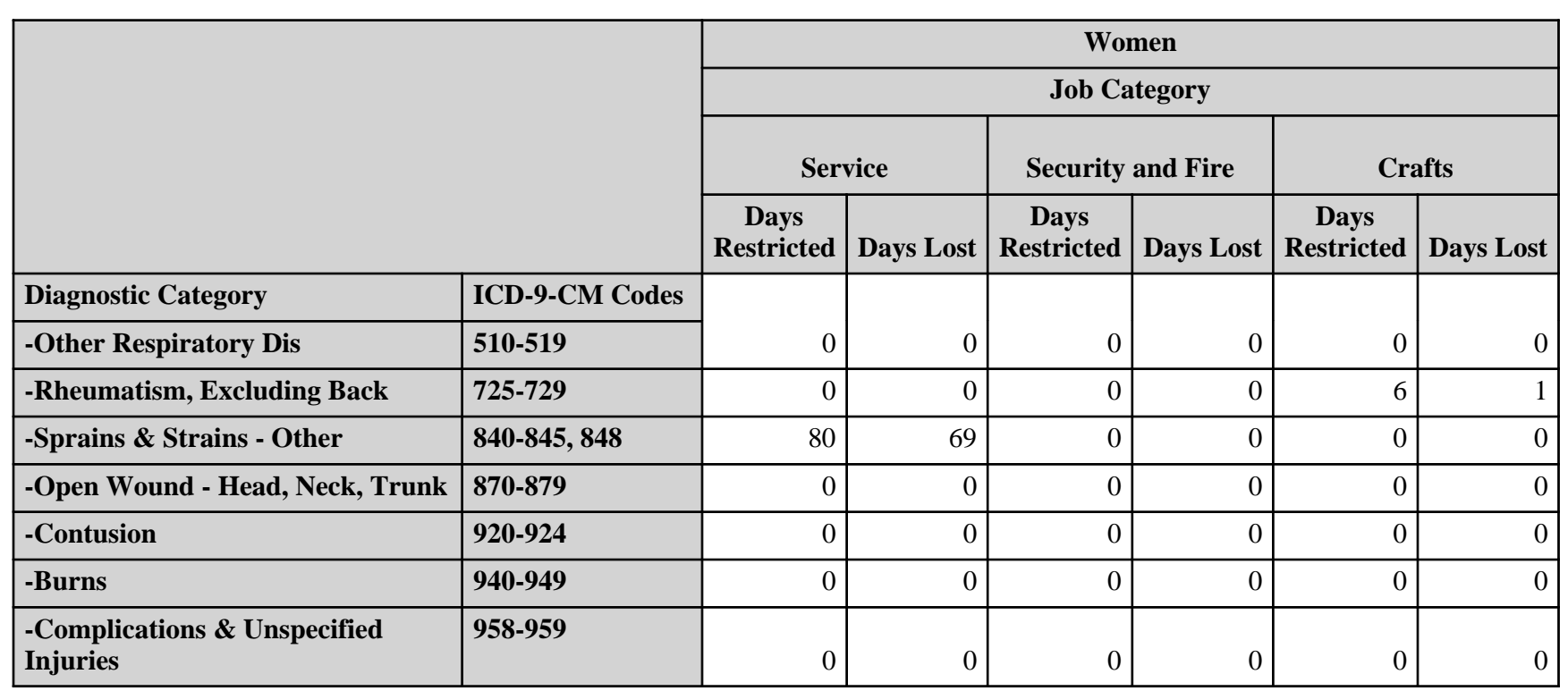

*OSHA events with >1 ICD-9-CM code in the same diagnostic category were counted only once. Only those diagnostic categories and gender/job category combinations with at least one occurrence appear in this table. 
Idaho National Laboratory 2007

OSHA Data

Appendix T. Number of Workdays Lost or with Restricted Activity in Each Diagnostic Category by Gender and Job Category*

\begin{tabular}{|c|c|c|c|c|c|c|c|c|c|}
\hline & & \multicolumn{8}{|c|}{ Men } \\
\hline & & \multicolumn{8}{|c|}{ Job Category } \\
\hline & & \multicolumn{2}{|c|}{ Professional } & \multicolumn{2}{|c|}{$\begin{array}{l}\text { Administrative } \\
\text { Support }\end{array}$} & \multicolumn{2}{|c|}{$\begin{array}{l}\text { Technical } \\
\text { Support }\end{array}$} & \multicolumn{2}{|c|}{ Service } \\
\hline & & $\begin{array}{c}\text { Days } \\
\text { Restricted }\end{array}$ & $\begin{array}{l}\text { Days } \\
\text { Lost }\end{array}$ & $\begin{array}{c}\text { Days } \\
\text { Restricted }\end{array}$ & $\begin{array}{l}\text { Days } \\
\text { Lost }\end{array}$ & \begin{tabular}{c|} 
Days \\
Restricted
\end{tabular} & $\begin{array}{l}\text { Days } \\
\text { Lost }\end{array}$ & $\begin{array}{c}\text { Days } \\
\text { Restricted }\end{array}$ & $\begin{array}{l}\text { Days } \\
\text { Lost }\end{array}$ \\
\hline Diagnostic Category & ICD-9-CM Codes & & & & & & & & \\
\hline -Disorders of Eye & $360-379$ & 0 & 0 & 0 & 0 & 0 & 0 & 0 & 0 \\
\hline -Other Respiratory Dis & $510-519$ & 0 & 0 & 0 & 0 & 0 & 0 & 0 & 0 \\
\hline -Dorsopathies & $720-724$ & 0 & 0 & 0 & 0 & 0 & 0 & 4 & 17 \\
\hline -Fracture - Skull & $800-804$ & 0 & 0 & 0 & 0 & 0 & 0 & 0 & 0 \\
\hline -Fracture - Upper Limb & 810-819 & 0 & 0 & 0 & 0 & 0 & 0 & 0 & 0 \\
\hline -Fracture - Lower Limb & $820-829$ & 0 & 0 & 0 & 180 & 0 & 0 & 0 & 0 \\
\hline -Sprains \& Strains - Back & $846-847$ & 0 & 0 & 0 & 0 & 28 & 22 & 0 & 0 \\
\hline -Sprains \& Strains - Other & $840-845,848$ & 0 & 11 & 0 & 0 & 78 & 97 & 17 & 25 \\
\hline -Open Wound - Upper Limb & 880-887 & 0 & 0 & 0 & 0 & 0 & 0 & 0 & 0 \\
\hline -Superficial Injury & $910-919$ & 0 & 0 & 0 & 0 & 0 & 0 & 0 & 0 \\
\hline -Foreign Body Entering Orifice & 930-939 & 0 & 0 & 0 & 0 & 0 & 0 & 0 & 0 \\
\hline $\begin{array}{l}\text {-Complications \& Unspecified } \\
\text { Injuries }\end{array}$ & 958-959 & 0 & 0 & 0 & 0 & 0 & 0 & 0 & 0 \\
\hline -Toxic Effects - Non-medicinal & $980-989$ & 0 & 0 & 0 & 0 & 0 & 0 & 0 & 0 \\
\hline $\begin{array}{l}\text {-Unspecified Effects - External } \\
\text { Causes }\end{array}$ & $990-995$ & 0 & 0 & 0 & 0 & 0 & 0 & 0 & 0 \\
\hline
\end{tabular}

*OSHA events with >1 ICD-9-CM code in the same diagnostic category were counted only once. Only those diagnostic categories and gender/job category combinations with at least one occurrence appear in this table. 
Idaho National Laboratory 2007

OSHA Data

Appendix T. Number of Workdays Lost or with Restricted Activity in Each Diagnostic Category by Gender and Job Category*

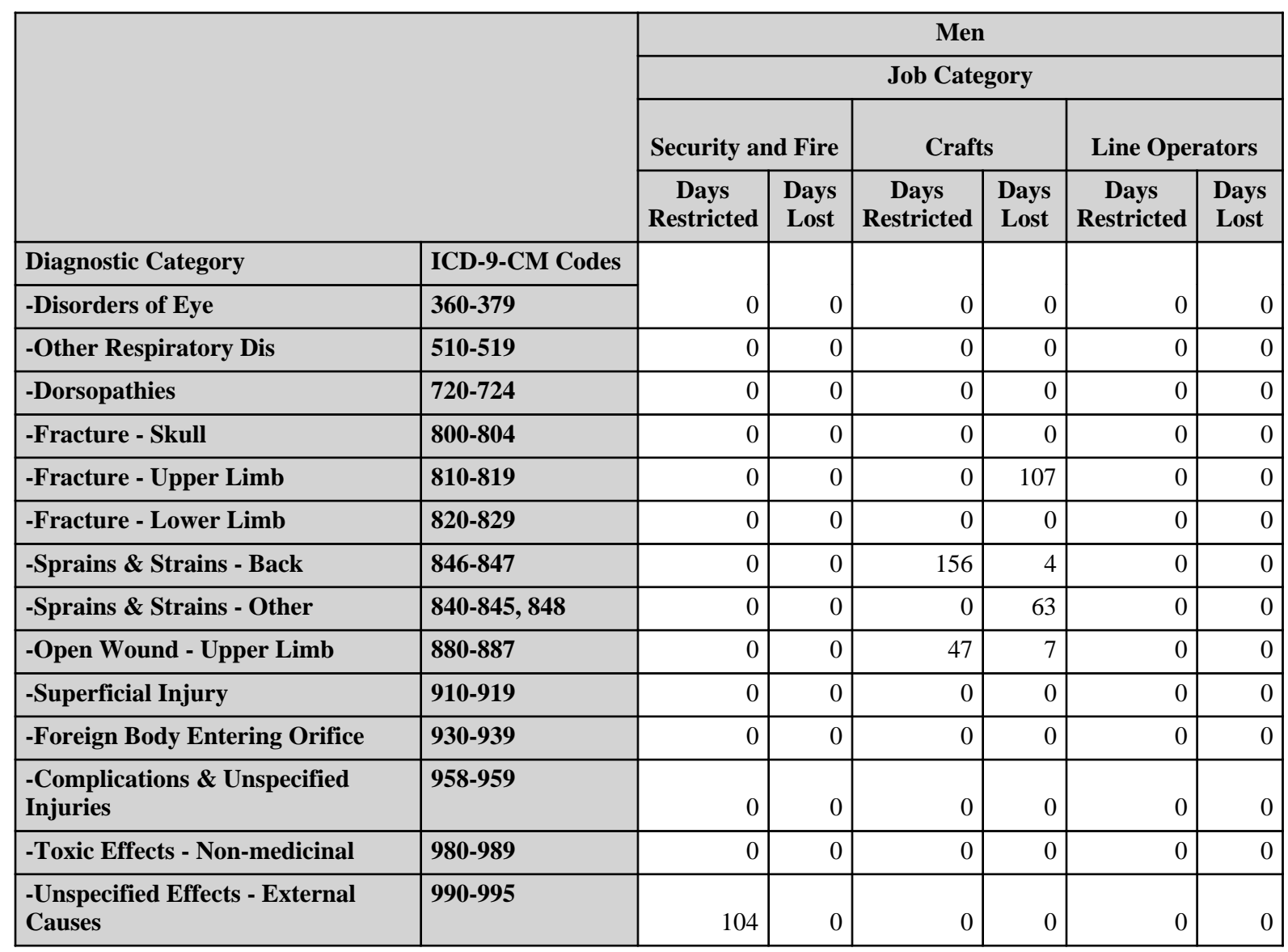

*OSHA events with >1 ICD-9-CM code in the same diagnostic category were counted only once. Only those diagnostic categories and gender/job category combinations with at least one occurrence appear in this table. 
Idaho National Laboratory 2007

OSHA Data

Appendix U. Number of Occurrences in Each Accident Category by Gender and Job Category*

\begin{tabular}{|c|c|c|c|c|}
\hline & & \multicolumn{3}{|c|}{ Women } \\
\hline & & \multicolumn{2}{|c|}{ Job Category } & \multirow[b]{2}{*}{ TOTAL } \\
\hline & & $\begin{array}{l}\text { Administrative } \\
\text { Support }\end{array}$ & $\begin{array}{l}\text { Technical } \\
\text { Support }\end{array}$ & \\
\hline Type of Accident & E CODES & \multirow[b]{2}{*}{1} & \multirow[b]{2}{*}{0} & \multirow[b]{2}{*}{1} \\
\hline Falls & E880-E888 & & & \\
\hline Other Accidents & E916-E928 & 1 & 1 & 2 \\
\hline
\end{tabular}

\begin{tabular}{|c|c|c|c|c|c|c|c|c|}
\hline & & \multicolumn{7}{|c|}{ Men } \\
\hline & & \multicolumn{6}{|c|}{ Job Category } & \multirow[b]{2}{*}{ TOTAL } \\
\hline & & Professional & $\begin{array}{c}\text { Administrative } \\
\text { Support }\end{array}$ & Service & $\begin{array}{l}\text { Security } \\
\text { and Fire }\end{array}$ & Crafts & $\begin{array}{c}\text { Line } \\
\text { Operators }\end{array}$ & \\
\hline Type of Accident & E CODES & \multirow[b]{2}{*}{1} & \multirow[b]{2}{*}{0} & \multirow[b]{2}{*}{0} & \multirow[b]{2}{*}{0} & \multirow[b]{2}{*}{0} & \multirow[b]{2}{*}{0} & \multirow[b]{2}{*}{1} \\
\hline Accidental Poisoning - Non-medicinal & E860-E869 & & & & & & & \\
\hline Falls & \begin{tabular}{|l|} 
E880-E888 \\
\end{tabular} & 1 & 0 & 0 & 0 & 0 & 0 & 1 \\
\hline $\begin{array}{l}\text { Submersion/Suffocation/Foreign } \\
\text { Bodies }\end{array}$ & E910-E915 & 0 & 0 & 0 & 0 & 1 & 0 & 1 \\
\hline Other Accidents & E916-E928 & 2 & 1 & 1 & 2 & 1 & 1 & 8 \\
\hline
\end{tabular}

*Only those accident types and gender/job category combinations with at least one occurrence appear in this table. 
Idaho National Laboratory 2007

OSHA Data

Appendix V. Number of Workdays Lost or with Restricted Activity in Each Accident Category by Gender and Job Category*

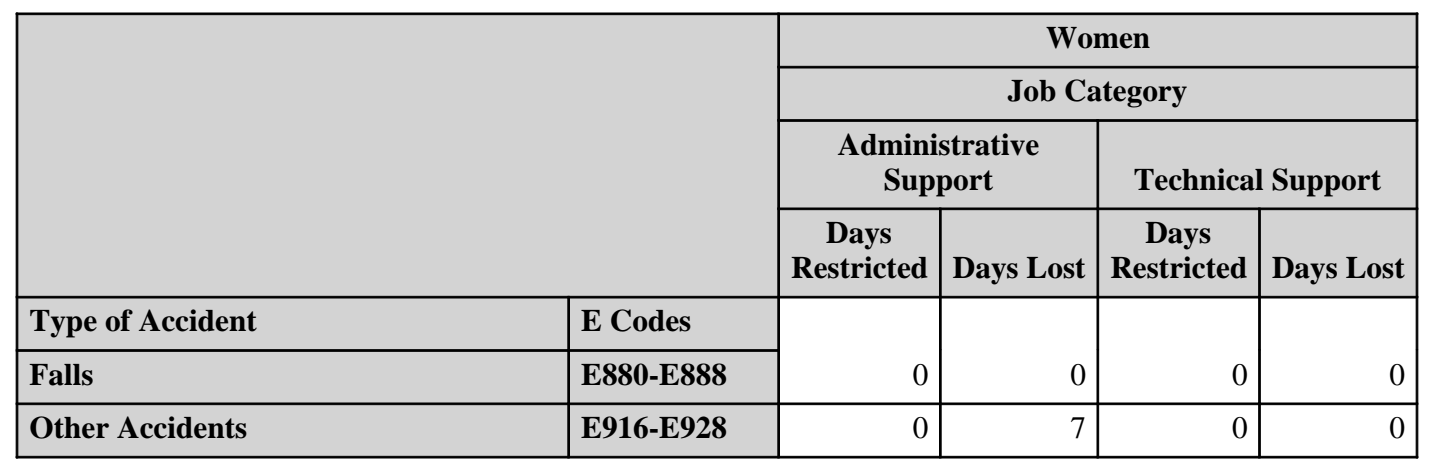

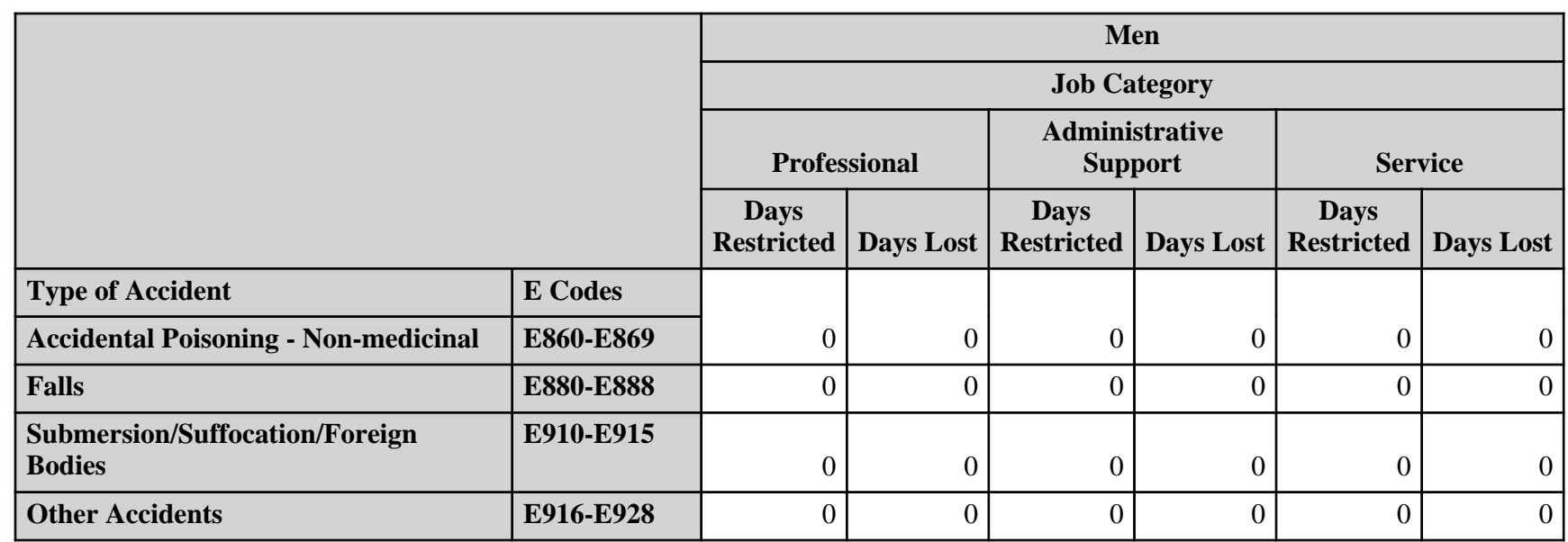

\begin{tabular}{|c|c|c|c|c|c|c|c|}
\hline & \multicolumn{6}{|c|}{ Men } \\
\hline & & \multicolumn{6}{|c|}{ Job Category } \\
\hline & & \multicolumn{2}{|c|}{ Security and Fire } & \multicolumn{2}{|c|}{ Crafts } & \multicolumn{2}{|c|}{ Line Operators } \\
\hline & & $\begin{array}{c}\text { Days } \\
\text { Restricted }\end{array}$ & Days Lost & \begin{tabular}{|c|} 
Days \\
Restricted
\end{tabular} & Days Lost & \begin{tabular}{|c|} 
Days \\
Restricted
\end{tabular} & Days Lost \\
\hline Type of Accident & E Codes & & & & & & \\
\hline Accidental Poisoning - Non-medicinal & E860-E869 & 0 & 0 & 0 & 0 & 0 & 0 \\
\hline Falls & E880-E888 & 0 & 0 & 0 & 0 & 0 & 0 \\
\hline $\begin{array}{l}\text { Submersion/Suffocation/Foreign } \\
\text { Bodies }\end{array}$ & E910-E915 & 0 & 0 & 0 & 0 & 0 & 0 \\
\hline Other Accidents & E916-E928 & 104 & 0 & 0 & 107 & 0 & 0 \\
\hline
\end{tabular}

*OSHA events with $>1 \mathrm{E}$ code in the same accident type were counted only once. Only those accident types and gender/job category combinations with at least one occurrence appear in this table. 
Idaho National Laboratory 2007

OSHA Data

Appendix W. Age-Adjusted OSHA Illness and Injury Rates by Diagnostic Category*

Part 1. Men

\begin{tabular}{|c|c|c|c|c|c|}
\hline & & $\begin{array}{l}\text { Number of } \\
\text { Diagnoses }\end{array}$ & $\begin{array}{l}\text { Age-Adjusted } \\
\text { Rate per } 1,000 * *\end{array}$ & $\begin{array}{c}\text { Lower } 95 \% \\
\text { Confidence } \\
\text { Limit per 1,000 }\end{array}$ & $\begin{array}{c}\text { Upper 95\% } \\
\text { Confidence } \\
\text { Limit per 1,000 }\end{array}$ \\
\hline Diagnostic Category & ICD-9-CM Code & \multirow[b]{2}{*}{2} & \multirow[b]{2}{*}{0.3} & \multirow[b]{2}{*}{0.1} & \multirow[b]{2}{*}{1.2} \\
\hline NERVOUS SYSTEM (NS) \& SENSE ORGANS & 320-389 & & & & \\
\hline -Disorders of Eye & $360-379$ & 2 & 0.3 & 0.1 & 1.2 \\
\hline RESPIRATORY SYSTEM & $460-519$ & 1 & 1.2 & 0.2 & 8.3 \\
\hline -Other Respiratory Dis & $510-519$ & 1 & 1.2 & 0.2 & 8.3 \\
\hline $\begin{array}{l}\text { MUSCULOSKELETAL \& CONNECTIVE } \\
\text { TISSUE }\end{array}$ & $710-739$ & 1 & 0.2 & 0.0 & 1.2 \\
\hline -Dorsopathies & $720-724$ & 1 & 0.2 & 0.0 & 1.2 \\
\hline INJURY \& POISONING & $800-999$ & 28 & 8.2 & 4.9 & 13.7 \\
\hline -Fracture - Skull & $800-804$ & 1 & 0.1 & 0.0 & 0.8 \\
\hline -Fracture - Upper Limb & 810-819 & 2 & 0.7 & 0.2 & 3.0 \\
\hline -Fracture - Lower Limb & $820-829$ & 3 & 0.3 & 0.1 & 1.0 \\
\hline -Sprains \& Strains - Back & 846-847 & 4 & 1.5 & 0.5 & 4.2 \\
\hline -Sprains \& Strains - Other & $840-845,848$ & 5 & 1.4 & 0.5 & 3.8 \\
\hline -Open Wound - Upper Limb & 880-887 & 7 & 1.9 & 0.5 & 6.6 \\
\hline -Superficial Injury & 910-919 & 1 & 0.1 & 0.0 & 0.8 \\
\hline -Foreign Body Entering Orifice & 930-939 & 1 & 0.2 & 0.0 & 1.2 \\
\hline -Toxic Effects - Non-medicinal & 980-989 & 3 & 1.8 & 0.5 & 7.2 \\
\hline -Complications of Surgical/Medical Care & 996-999 & 1 & 0.1 & 0.0 & 0.8 \\
\hline Total & & 32 & 9.8 & 6.0 & 16.0 \\
\hline
\end{tabular}

* Only those diagnostic categories with at least one occurrence appear in this table.

**Standardized to age distribution of 2000 U.S. population. 
Idaho National Laboratory 2007

OSHA Data

Appendix W. Age-Adjusted OSHA Illness and Injury Rates by Diagnostic Category*

Part 2. Women

\begin{tabular}{|c|c|c|c|c|c|}
\hline & & $\begin{array}{l}\text { Number of } \\
\text { Diagnoses }\end{array}$ & $\begin{array}{c}\text { Age-Adjusted } \\
\text { Rate per } 1,000 * *\end{array}$ & $\begin{array}{c}\text { Lower } 95 \% \\
\text { Confidence } \\
\text { Limit per 1,000 }\end{array}$ & $\begin{array}{c}\text { Upper 95\% } \\
\text { Confidence } \\
\text { Limit per 1,000 }\end{array}$ \\
\hline Diagnostic Category & ICD-9-CM Code & & & & \\
\hline RESPIRATORY SYSTEM & $460-519$ & 1 & 0.5 & 0.1 & 3.3 \\
\hline -Other Respiratory Dis & $510-519$ & 1 & 0.5 & 0.1 & 3.3 \\
\hline $\begin{array}{l}\text { MUSCULOSKELETAL \& CONNECTIVE } \\
\text { TISSUE }\end{array}$ & $710-739$ & 1 & 0.4 & 0.1 & 2.6 \\
\hline -Rheumatism, Excluding Back & $725-729$ & 1 & 0.4 & 0.1 & 2.6 \\
\hline INJURY \& POISONING & $800-999$ & 13 & 10.0 & 5.2 & 19.1 \\
\hline -Sprains \& Strains - Other & $840-845,848$ & 6 & 6.3 & 2.6 & 15.7 \\
\hline -Open Wound - Head, Neck, Trunk & 870-879 & 1 & 0.5 & 0.1 & 3.3 \\
\hline -Contusion & $920-924$ & 3 & 1.3 & 0.4 & 4.1 \\
\hline -Burns & 940-949 & 1 & 0.5 & 0.1 & 3.3 \\
\hline -Complications of Surgical/Medical Care & 996-999 & 2 & 1.4 & 0.3 & 6.5 \\
\hline Total & & 15 & 10.8 & 5.9 & 19.9 \\
\hline
\end{tabular}

*Only those diagnostic categories with at least one occurrence appear in this table.

**Standardized to age distribution of 2000 U.S. population. 
Idaho National Laboratory 2007

OSHA Data

Appendix W. Age-Adjusted OSHA Illness and Injury Rates by Diagnostic Category*

Part 3. Men and Women

\begin{tabular}{|c|c|c|c|c|c|}
\hline & & $\begin{array}{l}\text { Number of } \\
\text { Diagnoses }\end{array}$ & $\begin{array}{c}\text { Age-Adjusted } \\
\text { Rate per 1,000** }\end{array}$ & $\begin{array}{c}\text { Lower } 95 \% \\
\text { Confidence } \\
\text { Limit per } 1,000\end{array}$ & $\begin{array}{c}\text { Upper } 95 \% \\
\text { Confidence } \\
\text { Limit per 1,000 }\end{array}$ \\
\hline Diagnostic Category & ICD-9-CM Code & \multirow[b]{2}{*}{2} & \multirow[b]{2}{*}{0.2} & \multirow[b]{2}{*}{0.1} & \multirow[b]{2}{*}{0.9} \\
\hline NERVOUS SYSTEM (NS) \& SENSE ORGANS & 320-389 & & & & \\
\hline -Disorders of Eye & $360-379$ & 2 & 0.2 & 0.1 & 0.9 \\
\hline RESPIRATORY SYSTEM & $460-519$ & 2 & 0.9 & 0.2 & 5.1 \\
\hline -Other Respiratory Dis & $510-519$ & 2 & 0.9 & 0.2 & 5.1 \\
\hline $\begin{array}{l}\text { MUSCULOSKELETAL \& CONNECTIVE } \\
\text { TISSUE }\end{array}$ & $710-739$ & 2 & 0.2 & 0.1 & 0.9 \\
\hline -Dorsopathies & $720-724$ & 1 & 0.1 & 0.0 & 0.9 \\
\hline -Rheumatism, Excluding Back & 725-729 & 1 & 0.1 & 0.0 & 0.6 \\
\hline INJURY \& POISONING & $800-999$ & 41 & 8.1 & 5.5 & 12.1 \\
\hline -Fracture - Skull & $800-804$ & 1 & 0.1 & 0.0 & 0.6 \\
\hline -Fracture - Upper Limb & 810-819 & 2 & 0.6 & 0.1 & 2.3 \\
\hline -Fracture - Lower Limb & $820-829$ & 3 & 0.3 & 0.1 & 0.8 \\
\hline -Sprains \& Strains - Back & $846-847$ & 4 & 1.0 & 0.4 & 2.9 \\
\hline -Sprains \& Strains - Other & $840-845,848$ & 11 & 2.3 & 1.2 & 4.4 \\
\hline -Open Wound - Head, Neck, Trunk & 870-879 & 1 & 0.1 & 0.0 & 0.9 \\
\hline -Open Wound - Upper Limb & 880-887 & 7 & 1.3 & 0.4 & 4.5 \\
\hline -Superficial Injury & 910-919 & 1 & 0.1 & 0.0 & 0.6 \\
\hline -Contusion & $920-924$ & 3 & 0.3 & 0.1 & 1.1 \\
\hline -Foreign Body Entering Orifice & 930-939 & 1 & 0.1 & 0.0 & 0.9 \\
\hline -Burns & 940-949 & 1 & 0.1 & 0.0 & 0.9 \\
\hline -Toxic Effects - Non-medicinal & 980-989 & 3 & 1.2 & 0.3 & 4.8 \\
\hline -Complications of Surgical/Medical Care & 996-999 & 3 & 0.5 & 0.1 & 1.9 \\
\hline Total & & 47 & 9.5 & 6.5 & 13.8 \\
\hline
\end{tabular}

* Only those diagnostic categories with at least one occurrence appear in this table.

**Standardized to age distribution of 2000 U.S. population. 